\title{
Abstracts of poster presentations at the 2021 virtual annual meeting of the Canadian Society for Pharmaceutical Sciences.
}

In 2021, the Canadian Society for Pharmaceutical Sciences (CSPS) partnered with the Pharmaceutical Society of Japan (PSJ) and the Canadian Chapter of Controlled Release Society (CC-CRS) to present its annual meeting in the virtual style. Keynote speakers included Professor Michael Houghton - 2020 Nobel Laureate, Director of Li Ka Shing Applied Virology Institute (LKSAVI), Professor, Department of Medical Microbiology \& Immunology, University of Alberta, and Professor Yoshiharu Matsuura, Director, Center for Infectious Diseases Education and Research (CIDER), Osaka University, Japan.

The meeting program is found at https://pheedloop.com/csps2021/site/home/

General Presentations. Pages 1s-60s

Supplement: GSK/CSPS undergraduate students research award presentations scheduled to be presented at the cancelled 2020 annual CSPS meeting. Pages 61s-66s

\section{General Presentations:}

\section{Direct vasorelaxant effects of 22 benzodiazepines and 2 non-benzodiazepines hypnotics}

$\underline{\text { Satomi Kagota, }},{ }^{1}$ Kana. Maruyama-Fumoto,${ }^{2}$ Kana. Morikawa, ${ }^{3}$ Hirotake. Ishida, ${ }^{4}$ Junko. Chimoto, ${ }^{4}$ Shizuo. Yamada, ${ }^{5}$ Kazumasa. Shinozuka, ${ }^{6}$

${ }^{1}$ Professor, Department of Pharmacology II, School of Pharmacy and Pharmaceutical Sciences, Mukogawa Women's University; Institute for Bioscience, Mukogawa Women's University, Nishinomiya, Japan; ${ }^{2}$ Department of Pharmacology II, School of Pharmacy and Pharmaceutical Sciences, Mukogawa Women's University, Canada; ${ }^{3}$ Department of Pharmacology II, School of Pharmacy and Pharmaceutical Sciences, Mukogawa Women's University, Canada; ${ }^{4}$ Center for Pharma-Food Research, Graduate School of Pharmaceutical Sciences, University of Shizuoka, Shizuoka, Canada; ${ }^{5}$ Professor, Center for Pharma-Food Research, Graduate School of Pharmaceutical Sciences, University of Shizuoka, Shizuoka, Canada; ${ }^{6}$ Professor, Department of Pharmacology II, School of Pharmacy and Pharmaceutical Sciences, Mukogawa Women's University, Canada

Purpose: Benzodiazepine (BDZ) and non-BDZ hypnotics are commonly used for the management of chronic insomnia. They cause adverse effects, including headache, dizziness, and palpitations. Moreover, long-term BDZ therapy reduces blood pressure and increases the risk of developing orthostatic hypotension. The underlying mechanism of these reactions may be that such hypnotics have direct vasorelaxant effects. The present study aimed to test this hypothesis by evaluating the possible vasorelaxant effects of $22 \mathrm{BDZs}$ and 2 non-BDZs in phenylephrine-constricted rat arteries. Methods: Arterial rings were prepared from the thoracic aorta of male Wistar rats. Phenylephrine was applied to the rings to induce constriction, and BDZs and non-BDZs were added to the constricted rings. Arterial ring response was determined using the myograph method. In addition, gamma-aminobutyric acid type A (GABA) receptor binding assay was performed using $\left[{ }^{3} \mathrm{H}\right]$ flunitrazepam. Results: At a concentration of $10 \mu \mathrm{M}$, zolpidem relaxed the arterial rings by more than $80 \%$; diazepam, estazolam, etizolam, and tofisopam relaxed the rings by $60-70 \%$; and alprazolam, bromazepam, brotizolam, chlordiazepoxide, clobazam, clonazepam, clorazepate, ethyl loflazepate, flunitrazepam, flurazepam, lorazepam, lormetazepam, midazolam, nimetazepam, nitrazepam, oxazepam, temazepam, and triazolam as well as zaleplon relaxed the rings by less than $50 \%$. Removal of 
the endothelium and treatment with a nitric oxide (NO) synthase antagonist inhibited the vasorelaxant response to zolpidem, diazepam, etizolam, and tofisopam, while flumazenil treatment did not. Moreover, vasodilation induced at $10 \mu \mathrm{M}$ did not correlate with GABA receptor binding affinity for 23 drugs, except for tofisopam, which had a low binding affinity at less than $100 \mu \mathrm{M}$. Conclusion: This study demonstrated that many BDZ and non-BDZ hypnotics cause vasorelaxation via endothelial NO-dependent pathways, but that this is not mediated by GABA receptor activation. The direct vasodilatory effects of these drugs may be the underlying mechanism of their abovementioned side effects.

\section{Understanding the Mesenchymal Niche in Breast cancer}

Maryam Jama, ${ }^{1}$ Natchanon Kitcharoenroen, ${ }^{2}$ Pam Collier, ${ }^{3}$ Ali A. Ritchie, ${ }^{4}$ Marian Meakin, ${ }^{5}$ Philip A. Clarke, ${ }^{6}$ Anna Grabowska, ${ }^{7}$

${ }^{1}$ Lab Technician, University of Alberta, Canada; ${ }^{2}$ Graduate, ${ }^{3}$ Research Technician, ${ }^{4}$ Senior in vivo research manager, ${ }^{5}$ Technician, ${ }^{6}$ Technical Services Operations Manager, ${ }^{7}$ Professor of Cancer microenvironment, University of Nottingham, Nottingham. UK

Purpose: The stroma constitutes of Mesenchymal stem cells (MSCs), a promising therapeutic target for cancer treatment, however the role of MSCs in TME is controversial(1). Objective: To characterize the stroma in four Patient-Derived Xenograft (PDX) models; Triple negative and Luminal A co-engrafted with MSCs and without MSCs. Methods: In situ hybridization and bioluminescent imaging was used to localise and quantify MSCs, which had been lentivirally-transduced with the luciferase gene. H\&Es were used to identify stromal and non-stromal areas within the xenografts, and immunohistochemistry (IHC) staining to stain for several phenotypic markers. Results: After the co-engraftment of luciferase labeled human MSC in PDX, Luciferase expression increased over time. In TNBC PDX with MSC co-engraftment, the nonstromal area had 18\% CD34-positive cells. Within the stromal area, there were 30\% -sma-positive cells. Stromal and non-stromal areas had 45\% Vimentin-positive cells. In the TNBC PDX without MSCs coengraftment, the stromal area had 5\% Vimentin, 25\% CD34, and 20\%--sma-positive cells. There were more FSP positive cells compared to without MSC co-engraftment. In the luminal A PDX with MSC coengraftment, the non-stromal area had 25\% Vimentin-positive cells. The Stromal area had 10\% -sma and $15 \%$ CD34-positive cells. For Luminal A PDX without MSC co-engraftment $65 \%$ of cells were Vimentinpositive (in both stroma and non stromal areas). The stromal area had 20\% -sma-positive cells. The nonstromal area had 10\% CD34-positive cells. In Luminal A PDX, MSCs co-engraftment did not make a difference in FSP expression. Lastly, FSP expression was predominantly in the non-stromal area, for both PDXs. Conclusion: MSCs after co-engraftment acquired some different phenotypes. MSCs enhance Epithelial to mesenchymal transition in TNBC, but reduce it in Luminal A. Future studies such as trafficking engrafted human MSCs by fluorescent imaging is needed to enable tracking of individual cells over time and more detailed examination of their phenotype.

\section{Investigating the anticancer activities of novel SHP2 inhibitors against Triple-Negative Breast Cancer}

Anna Jutla ${ }^{1}$, Marawan Ahmed ${ }^{1}$, Jitendra Kumar ${ }^{2}$, Yasser Tabana ${ }^{1}$, Tae Chul moon ${ }^{1}$, Michael Overduin ${ }^{2}$ and Khaled H. Barakat ${ }^{1,3 *}{ }^{1}$ Faculty of Pharmacy and Pharmaceutical Sciences, University of Alberta, Canada.

${ }^{2}$ Department of Biochemistry, Faculty of Medicine and Dentistry, University of Alberta, Canada. ${ }^{3} \mathrm{Li} \mathrm{Ka}$ Shing Institute of Virology, University of Alberta, Canada.

Purpose: Src homology region 2 (SH2) domain-containing protein tyrosine phosphatase-(SHP2) is a cytoplasmic protein tyrosine phosphatase (PTP) which plays a vital role in Triple-Negative Breast Cancer (TNBC) progression. SHP2 mediates TNBC cell proliferation, migration, and metastasis by involving several intracellular oncogenic signaling pathways. It has been shown that tumor progression can be 
suppressed by suppressing SHP2's activity; hence, SHP2 can be used as a potential target for cancer therapy. Our lab has recently identified novel allosteric SHP2 inhibitors $(\mathrm{C} 8, \mathrm{~B} 9, \mathrm{C} 2)$ through in silico structure-based virtual screening of over 7-million compound libraries from the MolPort database. Methods: We applied various computational techniques, including pharmacophore search, docking, molecular dynamics and absolute binding free energy calculations to retrieve a list of potential SHP2 inhibitors. The narrowed potential inhibitors were validated by surface plasmon resonance (SPR). Preclinical investigation with in vitro apoptotic in TNBC cells and IL-2 production assays in peripheral blood mononuclear cell (PBMC) were conducted to study the effect of the compounds on TNBC. Results: SHP2 inhibitors showed strong binding in SPR where C8 exhibited the best binding affinity with a $\mathrm{Kd}$ of $83 \mathrm{nM}$ and the weakest is $\mathrm{C} 2$ with a $\mathrm{Kd}$ of $154 \mathrm{nM}$. C8 induces apoptosis as concentration increases in TNBC cells and induced IL-2 production with 570\% over the control in the concentration of $20 \mu \mathrm{g} / \mathrm{mL}$. Conclusion: Our research potentially leads to the identification of a novel SHP2 small molecule inhibitor with its anticancer mechanism against TNBC.

\section{The effects of elevated glucose on amylin content of islet-derived extracellular vesicles - implication in diabetes therapy}

Olia Katchanovski, ${ }^{1}$ Georges Grau, ${ }^{2}$ Elham. Hosseini-Beheshti, ${ }^{3}$ Lucy Marzban, ${ }^{4}$

${ }^{1}$ Undergraduate Student, University of Manitoba, Winnipeg, Canada

${ }^{2}$ Professor, University of Sydney, Canada

${ }^{3}$ Lecturer, University of Sydney, Canada

${ }^{4}$ Associate Professor, College of Pharmacy, University of Manitoba, Canada

Purpose: Type 2 diabetes (T2D) is characterized by peripheral insulin resistance and reduced beta-cell mass. Islet amyloid, formed by aggregation of human islet amyloid polypeptide (hIAPP; amylin), contributes to progressive beta-cell death in T2D. The cellular mechanisms underlying islet amyloid formation are still unclear. In this study, we examined the potential role of islet-derived extracellular vesicles $(\mathrm{EV})$ in clearance of amylin and its aggregates from islet beta cells and amyloid formation. Methods: Human islets isolated from cadaveric pancreatic donors $(n=4)$ and wild-type or hIAPP-expressing $\left(\mathrm{hIAPP}^{+}\right)$transgenic mouse islets $(\mathrm{n}=4$ /group) were cultured in normal or elevated glucose to form amyloid similar to that observed in T2D. EV (exosomes) were isolated from culture medium by centrifugation/ultracentrifugation and purified EV were analyzed by nanoparticle tracking analysis. EV markers and soluble and aggregated amylin were detected by Western blot analysis and double immunogold transmission electron microscopy. Results: Amyloid formation in human islets cultured with elevated glucose closely correlated with progressive beta-cell death. Mature and immature amylin were detectable in EV released from human islets cultured in normal or elevated glucose but were markedly higher in elevated glucose. Similarly, EV released from amyloid forming hIAPP ${ }^{+}$mouse islets had higher amylin content than wild-type islet-derived EV. Moreover, EV released from human islets cultured in elevated glucose contained beta-cell toxic amylin aggregates. Similarly, amylin aggregates were present in EV derived from amyloid forming hIAPP $^{+}$islets but not wild-type islets. Conclusion: Our data show that amylin forms are present in islet-derived EV and that diabetic condition increases amylin and its aggregates in EV released from islets, suggesting that islet-derived EV may play a key role in amyloid formation in T2D. Modulation of amylin clearance by islet-derived EV may provide a new therapeutic strategy to preserve beta cells in T2D by reducing amyloid-induced beta-cell death. 


\section{A polyphenol in functional foods improves cancer immunotherapy by modulating the gut microbiome}

$\underline{\text { Reilly Pidgeon }},{ }^{1}$ Meriem Messaoudene, ${ }^{2}$ Suraya Yasmine, ${ }^{3}$ Corentin Richard, ${ }^{2}$ Emmanuel Gonzalez, ${ }^{4}$ David Kysela, ${ }^{5}$ Yves Brun, ${ }^{6}$ André Marette, ${ }^{7}$ Bertrand Routy, ${ }^{8}$ Bastien Castagner, ${ }^{9}$

${ }^{1}$ Graduate Student, Department of Pharmacology \& Therapeutics, Faculty of Medicine and Health Sciences, McGill University; Montreal, Canada, Montreal, Canada

${ }^{2}$ Post-Doctoral Researcher, University of Montreal Research Center (CRCHUM); Montreal, Canada, Canada

${ }^{3}$ Graduate Student, Department of Pharmacology \& Therapeutics, Faculty of Medicine and Health Sciences, McGill University; Montreal, Canada, Canada

${ }^{4}$ Bioinformatics Specialist, Canadian Centre for Computational Genomics, McGill University and Genome Quebec Innovation Center; Montreal, Canada, Canada

${ }^{5}$ Research Scientist, Faculté de Médecine, Département de Microbiologie, infectiologie et immunologie, Université de Montréal; Montréal, Canada, Canada

${ }^{6}$ Distinguished Professor, Faculté de Médecine, Département de Microbiologie, infectiologie et immunologie, Université de Montréal; Montréal, Canada, Canada

${ }^{7}$ Professor, Department of Medicine, Faculty of Medicine, Cardiology Axis of the Québec Heart and Lung Institute and Institute of Nutrition and Functional Foods, Laval University; Québec City, Canada, Canada

${ }^{8}$ Assistant Professor, Hematology-Oncology Division, Department of Medicine, University of Montreal Healthcare Centre (CHUM); Montreal, Canada, Canada

${ }^{9}$ Associate Professor, Department of Pharmacology \& Therapeutics, Faculty of Medicine and Health Sciences, McGill University; Montreal, Canada, Canada

Purpose: Immune checkpoint inhibitors (ICIs) have revolutionized the treatment of cancer; however, patient response rates remain poor. Recent investigations have shown that specific gut microbes are enriched in patients that respond to ICIs. Murine studies have shown that shifting a non-responder gut microbiota towards that of an ICI responder can restore ICI efficacy. Here, we identify the active compound in a polyphenol-rich functional food, camu-camu, that alters gut microbiota composition and improves ICI efficacy. Methods: Polyphenols in camu-camu were fractionated by reversed-phase chromatography, and their anti-cancer activity was evaluated in ICI-sensitive and ICI-resistant mouse models of cancer. To identify direct interactions between the isolated polyphenol and gut microbes, a fluorescent polyphenol conjugate (probe) was synthesized and incubated with microbiota samples from six healthy subjects. Fluorescent bacteria were then sorted by flow cytometry. $16 \mathrm{~S}$ rRNA gene sequencing was used to evaluate changes in bacterial abundance in vehicle- versus polyphenol-treated mice and in labeled versus control microbiota samples. To localize where the probe bound in bacteria, labeled bacterial isolates were imaged by epifluorescence microscopy. Results: Both camu-camu and the isolated polyphenol restored ICI efficacy in non-responder avatar mice. This effect was microbiome-dependent with enrichment of Ruminococcus species and Akkermansia muciniphila. The fluorescent polyphenol probe selectively labeled bacteria in microbiota samples. Strong labeling was confirmed with bacterial isolates from the labeled population. The fluorescent signal in bacterial isolates was localized at the periphery of the cell. Conclusion: We have identified a single polyphenolic compound in camu-camu that improves the efficacy of cancer immunotherapy through the gut microbiota. 


\section{The synthesis of oligonucleotide incorporating 2-methoxycarbonyl-6-aminopurine for post-synthetic} modifications

Jeongsu Lee, ${ }^{1}$ Hirotaka Murase,${ }^{2}$ Hayate Takasaki, ${ }^{3}$ Taniguchi Yosuke, ${ }^{4}$ Sasaki Shigeki,${ }^{5}$

${ }^{1}$ Research assistant, Nagasaki International University, Japan

${ }^{2}$ Research assistant professor, Nagasaki International University, Japan

${ }^{3}$ Student, Kyushu University, Japan

${ }^{4}$ Associate professor, Kyushu university, Japan

${ }^{5}$ professor, Nagasaki International University, Japan

Purpose: The nucleic acid labeling is a powerful tool in various and wide fields, e.g., in vivo imaging, distribution analysis, functional/structural research or detection using biotin-streptavidin interaction, and the convenient labeling based on post-synthetic method has been applied to reduce the multi-step reactions before the DNA synthesis. In this study, we propose a new structure and method for post-synthetic modifications of the purine DNA base. Methods: We designed the phosphoramidite precursor of 2methoxycarbonyl-6-aminopurine for post-synthetic modification at 2-position of purine base. After incorporation into oligomer by DNA synthesizer, the introduction of amino compounds to the 2-carbonyl group can be executed cleaving from the resin and deprotecting the synthesized oligonucleotide.Result: The 5'-dimethoxytrityl 3'-phosphoramidite precursor monomer was synthesized starting from 2'-

deoxyguanosine in 10 steps and successfully incorporated into the oligodeoxynucleotide by the standard in the standard phosphoramidite method. The resulting CPG support was treated with several amines followed by HPLC purification and following DMTr deprotection gave the amine-modified oligonucleotide in good yield. Conclusion: In this study, we designed and synthesized oligonucleotide which can be applied to the post-synthetic modifications at the 2-position of the purine base. Now, we are investigating the introduction of various functional groups or molecules to our oligonucleotide.

\section{Monocarboxylate transporter 12 (MCT12) contributes to process of creatine biosynthesis as an efflux transporter of creatine and guanidinoacetate in the kidney and liver}

Ryuta Jomura, ${ }^{1}$ Yu Tanno, ${ }^{2}$ Shin-ichi Akanuma, ${ }^{3}$ Yoshiyuki Kubo, ${ }^{4}$ Masanori Tachikawa, ${ }^{5}$ Ken-ichi Hosoya, ${ }^{6}$

${ }^{1}$ Graduate student, Department of Pharmaceutics, Graduate School of Medicine and Pharmaceutical Sciences, University of Toyama, Toyama, Japan

${ }^{2}$ Graduate student, Department of Pharmaceutics, Graduate School of Medicine and Pharmaceutical Sciences, University of Toyama, Japan

${ }^{3}$ Assistant professor, Department of Pharmaceutics, Graduate School of Medicine and Pharmaceutical Sciences, University of Toyama, Japan

${ }^{4}$ Associate professor, Department of Pharmaceutics, Graduate School of Medicine and Pharmaceutical Sciences, University of Toyama, Japan

${ }^{5}$ Professor, Graduate School of Biomedical Sciences, Tokushima University, Japan

${ }^{6}$ Professor, Department of Pharmaceutics, Graduate School of Medicine and Pharmaceutical Sciences, University of Toyama, Toyama, Japan

Purpose: Creatine (Cr)/phosphocreatine is energy-related compounds which have the ability of ATP buffering and has protection effect against cell damages. Understanding the mechanism of Cr biosynthesis can be beneficial for the regulation of $\mathrm{Cr}$ dynamics in the body. De novo $\mathrm{Cr}$ biosynthesis is known to be involved in renal proximal tubular epithelial cells (RPTECs) and hepatocytes. In RPTECs, guanidinoacetate (GAA), a precursor of $\mathrm{Cr}$, is biosynthesized and transported to hepatocytes. In hepatocytes, $\mathrm{Cr}$ is biosynthesized from GAA and released into the circulatory system. In the process of $\mathrm{Cr}$ biosynthesis, the 
efflux mechanism of Cr and GAA from RPTECs and hepatocytes has not been understood. Therefore, this study aimed to elucidate the efflux mechanism of GAA and $\mathrm{Cr}$ from RPTECs and hepatocytes. Methods Rat MCT12-mediated $\left[{ }^{14} \mathrm{C}\right] \mathrm{Cr}$ and $\left[{ }^{14} \mathrm{C}\right] \mathrm{GAA}$ transport was evaluated using Xenopus laevis oocyte expression system. Protein localization was evaluated using immunohistochemistry. Contribution of MCT12 to efflux of $\left[{ }^{14} \mathrm{C}\right] \mathrm{Cr}$ and $\left[{ }^{14} \mathrm{C}\right] \mathrm{GAA}$ was evaluated by RNA interference in primary cultured rat RPTECs and hepatocytes. Results: In transport studies using oocytes, MCT12-mediated uptake of $\left[{ }^{14} \mathrm{C}\right] \mathrm{Cr}$ and $\left[{ }^{14} \mathrm{C}\right]$ GAA was observed with a $\mathrm{Km}$ value with $240 \mu \mathrm{M}$ and $3.4 \mathrm{mM}$, respectively ${ }^{1}$. In addition, MCT12-mediated uptake was extracellular inorganic ion-independent. Moreover, MCT12 showed the ability of efflux of $\left[{ }^{14} \mathrm{C}\right] \mathrm{Cr}$ and $\left[{ }^{14} \mathrm{C}\right] \mathrm{GAA}$. In immunohistochemistry, MCT12 protein was localized on the sinusoidal membrane of hepatocytes. $\left[{ }^{14} \mathrm{C}\right] \mathrm{Cr}$ efflux form primary cultured hepatocytes was significantly decreased following MCT12 knockdown. $\left[{ }^{14} \mathrm{C}\right]$ GAA efflux form primary cultured RPTECs was also significantly decreased after MCT12 knockdown ${ }^{1}$. Since MCT12 is reported to be localized on the basal membrane of RPTECs ${ }^{2}$, MCT12 is suggested to be involved in GAA release from RPTECs. Conclusion: MCT12 is suggested to play the role in efflux of Cr and GAA in RPTECs and hepatocytes, respectively. Therefore, MCT12 contributes to the process of Cr biosynthesis.

\section{Development of aniline modified aminonebularine derivatives for the formation of anti-parallel type of triplex DNA}

Ryotaro Notomi,${ }^{1}$ Lei Wang, ${ }^{2}$ Sasaki Shigeki, ${ }^{3}$ Taniguchi Yosuke, ${ }^{4}$

${ }^{1}$ Doctoral course student, Kyushu university, Canada

${ }^{2}$ post doctor, Kyushu university, Japan

${ }^{3}$ professor, Nagasaki International University, Japan

${ }^{4}$ Associate professor, Kyushu university, Japan

Purpose: Triplex DNA is a high-ordered structure interacted between triplex-forming oligonucleotides (TFOs) and duplex DNA from the major groove side in the sequence specific manner. Triplex DNA is extremely useful biological tool, because it is possible to regulate the gene expression at the transcription step and to develop the duplex DNA targeting agents. However, the stable triplex DNA forming region by natural nucleic acids is limited to the sequence consisting of GC and AT base pairs. Therefore, we have developed artificial nucleoside analogues that can recognize CG and TA base pairs, which are called as mismatch sites. In this study, we design and synthesize novel artificial nucleoside analogues based on the aminonebularine (dAN) moiety to expand the triplex forming region. Methods: We designed aniline modified dAN derivatives (3-aniline-dAN) based on the dAN/CG base triplet, and synthesized by the coupling reaction between 2-chloro purine and 1,3-diamino-benzen. Then this compound was converted to the amidite unit. After synthesis of TFOs having 3-aniline-dAN by an automated DNA synthesizer, they were purified by HPLC and confirmed by the MALDI-TOF MS measurements. The triplex forming abilities of synthesized TFOs were evaluated by the non-denatured acrylamide gel-shift assay. Results: According to the association constant of triplex formations, the triplex DNA with artificial TFOs did not show high stability, but it was confirmed in various sequences. One of the reasons why the stability was not sufficient is considered to be the weak hydrogen bond donor capacity of aniline. Conclusion: We have succeeded in synthesizing 3-aniline-dAN as a new artificial nucleoside derivative and TFO containing it. There was no obvious selectivity for target base pair, but triplex DNA formation was confirmed. Currently, we are continuing to search for dAN derivatives including the optimal linker. 
(a)

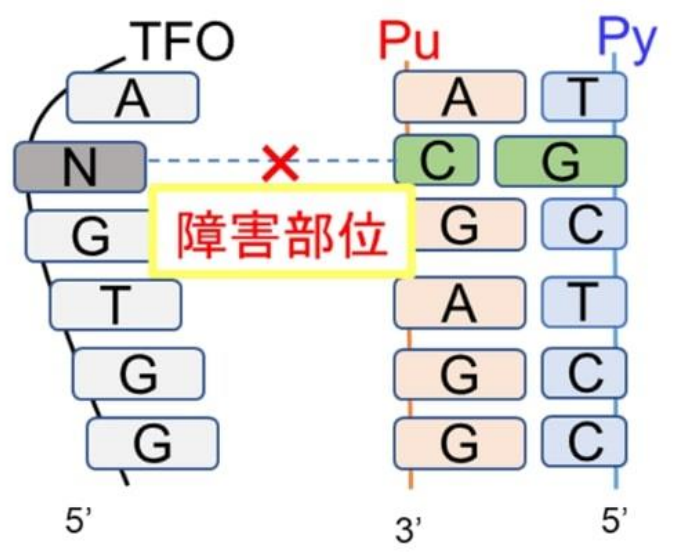

(b)

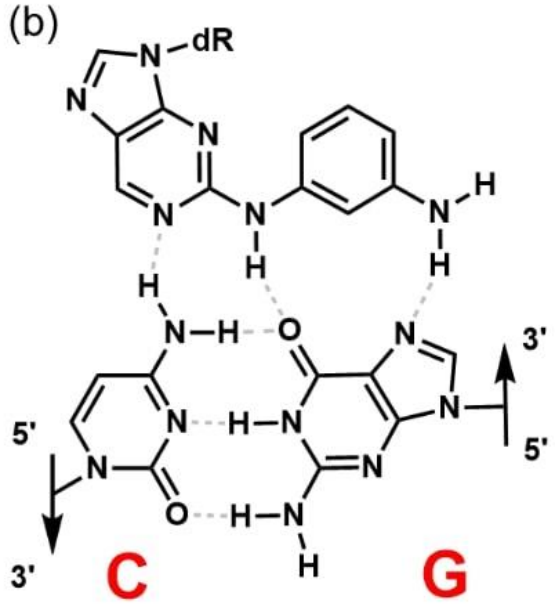

Figure : (a)Restriction of region by Triplex reverse site (b)Recognition style of 3-Aniline-dAN to CG base pair

\section{Development of 7-deaza-purine-1,3-diazaphenoxazine nucleotide derivative for the selective recognition of 8-oxo-2'-deoxyguanosine in DNA}

Ryoji Kawazoe, ${ }^{1}$ Yusuke Nagata, ${ }^{2}$ Ryo Miyahara,,${ }^{3}$ Takato Sakurada, ${ }^{1}$ Sasaki Shigeki, ${ }^{4}$ Taniguchi Yosuke, ${ }^{5}$

${ }^{1}$ Master student, graduated school of pharmaceutical science, Kyushu University, Canada

${ }^{2}$ Graduated of graduate school, graduated school of pharmaceutical science, Kyushu University, Canada

${ }^{3}$ Doctor student, graduated school of pharmaceutical science, Kyushu University, Canada

${ }^{4}$ Professor, Nagasaki International University, Japan

${ }^{5}$ Associate professor, Kyushu university, Japan

Purpose: Cellular DNA is continuously exposed to a variety of chemically reactive species. These species react with DNA to cause damaged nucleic acids. 8-Oxo-2'-deoxyguanosine (8-oxo-dG), the oxidative damaged nucleoside, is generated by the oxidation of 8-position of 2'-deoxyguanosine ( $\mathrm{dG}$ ). As 8-oxo-dG forms a base pair not only with $\mathrm{dC}$ but also with $\mathrm{dA}$, they induce transversion mutation from the GC to the TA base pair during DNA replication step. A number of studies have shown that the intracellular level of 8oxo-dG is related to diseases. However, since the position of occurrence in DNA is also understood to be an important factor in disease, the sequence-specific detection of 8-oxo-dG is challenging theme. In particular, selective and sequence-conservative detection of 8-oxo-dG without chemical treatment is a high demand. Recently, we have developed the adenosine-1,3-diazaphenoxazine triphosphate (dAdapTP) that is enzymatically incorporated into 8-oxo-dG in template DNA. Method In this study, we newly designed the 7-deaza-purine-1,3-diazaphenoxazine (7-deaza-Pdap) for selective recognition of 8-oxo-dG in DNA. And we synthesized its triphosphate and tested for single-nucleotide incorporation reaction using the primer strand, template DNA containing 8-oxo-dG, dG, dA, dC and T and Klenow Fragment. Results: The nucleoside part was estimated the coupling reaction between 6-iodo-7-deazapurine and Hoffer's chlorosugar. After that, the 7-deaza-Pdap was obtained by the Sonogashira coupling with phenoxazine part in a good yield. After conversion to the corresponding triphosphate compound, we evaluated the enzymatic reaction. As a result, no band with an extension could be observed. Unfortunately, we have tried various conditions and have not found efficient incorporation to the primer strand. Conclusion: We newly designed the non-natural purine nucleoside analogue, 7-deaza-Pdap. And we have successfully synthesized the 
triphosphate of 7-deaza-Pdap and tested for single-nucleotide incorporation reaction, that indicated that nitrogen atom at 7-position of the artificial nucleoside derivative was important in the enzymatic reaction.

\begin{tabular}{|c|c|c|c|c|c|c|c|}
\hline \multirow[b]{2}{*}{ Entry } & $x=o x$ & \multirow[b]{2}{*}{ Conc. of TP } & \multirow{2}{*}{ time } & \multicolumn{4}{|c|}{ 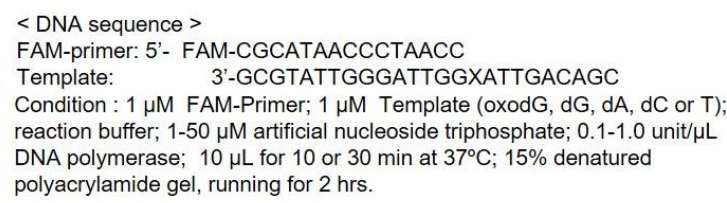 } \\
\hline & Polymerase & & & Entry & Polymerase & Conc. of TP & time \\
\hline 1 & 0.1 unit/ $\mu \mathrm{L} \mathrm{Kf}$ (exo-) & $50 \mu \mathrm{M}$ & $10 \mathrm{~min}$ & 8 & 1 unit/ $/ \mathrm{L} \mathrm{Kf}\left(\mathrm{exo}^{-}\right)$ & $50 \mu \mathrm{M}$ & $30 \mathrm{~min}$ \\
\hline 2 & 0.1 unit/ $/ \mu \mathrm{L}$ Kf (exo-) & $50 \mu \mathrm{M}$ & $30 \mathrm{~min}$ & 9 & 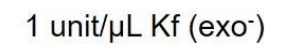 & $10 \mu \mathrm{M}$ & $30 \mathrm{~min}$ \\
\hline 3 & 0.1 unit/ $/ \mu \mathrm{L}$ Kf (exo-) & $10 \mu \mathrm{M}$ & $30 \mathrm{~min}$ & 10 & 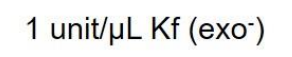 & $1 \mu \mathrm{M}$ & $30 \mathrm{~min}$ \\
\hline 4 & 0.1 unit/ $\mu \mathrm{L}$ Kf (exo-) & $1 \mu \mathrm{M}$. & $30 \mathrm{~min}$ & 11 & 0.1 unit $/ \mu \mathrm{L}$ Bst 3.0 & $50 \mu \mathrm{M}$. & $10 \mathrm{~min}$ \\
\hline 5 & 0.2 unit/ $\mu \mathrm{L}$ Kf (exo-) & $50 \mu \mathrm{M}$ & $30 \min$ & 12 & 0.1 unit $/ \mu L$ Bst 3.0 & $50 \mu \mathrm{M}$ & $30 \mathrm{~min}$ \\
\hline 6 & 0.2 unit/ $/ \mu \mathrm{L}$ Kf (exo-) & $10 \mu \mathrm{M}$. & $30 \mathrm{~min}$ & 13 & 0.1 unit/ $/ \mu \mathrm{L}$ Bsu & $50 \mu \mathrm{M}$. & $30 \mathrm{~min}$ \\
\hline 7 & 0.2 unit/ $\mu \mathrm{L} \mathrm{Kf}\left(\mathrm{exo}^{-}\right)$ & $1 \mu \mathrm{M}$ & $30 \mathrm{~min}$ & 14 & 0.1 unit $/ \mu \mathrm{L} \mathrm{Kf}\left(\mathrm{exo}^{+}\right)$ & $50 \mu \mathrm{M}$ & $30 \mathrm{~min}$ \\
\hline
\end{tabular}

Probing the anti-atherosclerotic potential of MPE-298, a cyclic azapeptide ligand of CD36/SR-B2 in hypercholesterolemic apolipoprotein E-deficient mice

$\underline{\text { Jade gauvin }},{ }^{1}$ Sylvie Marleau, ${ }^{2}$

${ }^{1}$ Student, université de Montréal, sainte-anne-des-plaines, Canada

${ }^{2}$ Professor, laboratory director, université de Montréal, Canada

Purpose:. Monocytes/macrophages scavenger receptor B2 (CD36/SR-B2), a receptor implicated in lipid cellular uptake, is known to play a key role in the development and pathogenesis of atherosclerosis. To underscore CD36/SR-B2 as a potential therapeutic target in atherosclerosis, cyclic azapeptide derivatives of growth hormone-releasing peptide-6 (GHRP-6) (His-D-Trp-Ala-TRP-D-Phe-Lys-NH $\mathrm{N}_{2}$ ), a known ligand of CD36/SR-B2, were developed. MPE-298, a potent macrocyclic azapeptide ligand of CD36, exhibited antiinflammatory activity in cell-based screening assays. Methods: Male apolipoprotein E deficient (apoE ${ }^{-/}$) mice were fed a high fat high cholesterol (HFHC) diet from 4 weeks of age (D12108, Research Diets, USA) and were treated with $300 \mathrm{nmol} \mathrm{MPE}-298 / \mathrm{kg}$ or vehicle $(0.9 \% \mathrm{NaCl})$ from 12 to 20 weeks of age to assess the effect of treatment on lesion progression. In addition, MPE-003, a linear azapeptide with a CD36 dependent anti-atherosclerotic effect (1), was used as a positive control, with the same dosage. Results: The Resulrs: show that, compared to the vehicle-treated group, MPE-298 reduced aortic arch lesion progression by $40 \%$ ( $p<0.0001$ ), which is comparable to the reduction obtained with the positive control group (42\%). Furthermore, a $53 \%$ reduction of IL- $1 \beta(\mathrm{p}=0.04)$ and a $43 \%$ reduction of TNF $(\mathrm{p}=0.03)$ plasma levels were observed, compared to vehicle-treated, HFHC-fed mice apoE ${ }^{-/}$. No significant change was observed in plasma cholesterol levels. Conclusion: This study shows that macrocyclic azapeptide MPE-298 reduced atherosclerotic lesion progression. That was associated with its anti-inflammatory activity but unlikely through its modulation of cholesterol metabolism. 


\title{
Sensitization of TRPA1 is involved in dysesthesia induced by blood flow reduction
}

\author{
$\underline{\text { Kanako So }},{ }^{1}$ Haruka Hiyama, ${ }^{2}$ Yuichi Yano,${ }^{2}$ Takayuki Nakagawa, ${ }^{3}$ Shuji Kaneko, ${ }^{4}$ \\ ${ }^{1}$ Junior Assistant Professor, Kyoto University, Graduate School of Pharmaceutical Sciences, Canada \\ ${ }^{2}$ Graduate Student, Kyoto University, Graduate School of Pharmaceutical Sciences, Canada \\ ${ }^{3}$ Associate Professor, Kyoto University Hospital, Canada \\ ${ }^{4}$ Professor, Kyoto University, Graduate School of Pharmaceutical Sciences, Canada
}

Purpose: Dysesthesia is an unpleasant abnormal sensation, often accompanied by peripheral neuropathy or vascular impairment. In this study, we examined the roles and molecular mechanism of redox-sensitive TRPA1 in dysesthesia by using two animal models of dysesthesia following reductions in blood flow, transient hindlimb ischemia/reperfusion model and hindlimb ischemia model. Methods: Ttransient hindlimb ischemia/reperfusion was induced by tightly ligating a left hindlimb of mice for $1 \mathrm{~h}$, and then removed the ligature.Hindlimb ischemia was induced by tightly ligating external iliac artery and vein of mice. The skin blood flow of the plantar surface of the hindpaw was measured by a laser speckle blood flow analysing system. Cold sensitivity was measured with a hot/cold-plate analgesimeter at $5^{\circ} \mathrm{C}$. Escape behaviors were observed for $60 \mathrm{~s}$ and scored. Mechanical sensitivity was assessed by measuring the pawwithdrawal threshold using calibrated von Frey filaments. Changes of intracellular calcium concentration were evaluated with fluorometric $\mathrm{Ca}^{2+}$ imaging analysis of Fura-2AM. Results: Transient hindlimbischemia/reperfusion evoked spontaneous licking, which is accompanied by mechanical hypoesthesia. The licking was inhibited by ROS scavengers or TRPA1 antagonist/deficiency. In the hindlimb ischemia models, the nocifensive behaviors induced by intraplantar injection of a TRPA1 agonist, and escape behavioral scores in response to cold stimulation were increased. These behaviors were reversed by TRPA1 antagonist/deficiency and administration of tadalafil. In human TRPA1-expressing cells and cultured mouse dorsal root ganglion neurons,pretreatment with hypoxia enhanced $\mathrm{H} 2 \mathrm{O} 2$-induced TRPA1 responses. The hypoxia-induced TRPA1 sensitization to $\mathrm{H} 2 \mathrm{O} 2$ was inhibited by the overexpression of a catalytically-dead mutant of prolyl hydroxylase (PHD) 2. Conclusion: Dysesthesia following blood flow reduction is caused by ROS-evoked activation of TRPA1 sensitized by hypoxia through inhibiting PHDmediated hydroxylation of a proline residue in TRPA1.

\section{Assessing the effects of vitamin $E$ acetate and cannabinoids on lung surfactant}

\author{
Nicolas Van Bavel, ${ }^{1}$ Elmar Prenner, ${ }^{2}$ Raimar Löbenberg, ${ }^{3}$ Patrick Lai, ${ }^{4}$ \\ ${ }^{1}$ Master's student, University of Calgary, Canada \\ ${ }^{2}$ Professor, University of Calgary, Canada \\ ${ }^{3}$ Professor, University of Alberta, Edmonton, Canada \\ ${ }^{4}$ Research Assistant, University of Calgary, Canada
}

Purpose: Vitamin E acetate was recently identified to be one of the main components of e-cigarette/vape induced lung injury [1]. The mechanism for impairment of lung function is still unclear, however one potential target is the lung surfactant. This is a monolayer of lipids and proteins responsible for reducing surface tension in alveoli. Disruption of this function results in the collapse of the alveolar sacs. We have developed a lung surfactant model system to investigate the impact of vitamin E acetate. Methods: Surface pressure-area isotherms were measured for the model system at the air-water interface to assess film stability, while Brewster angle microscopy was used to visualize potential changes to the lateral organization in real time. Model films with and without vitamin E acetate were cycled between low and high pressures to mimic the breathing cycles. Interactions with THC and CBD were undertaken to compare their effect to the additive and to assess potential interactions between the additive and the cannabinoids. 
Results: Preliminary results show a potential for vitamin E acetate, CBD, and THC to destabilize the films, presumably by material transfer into the subphase. This effect was seen to the greatest extent in the presence vitamin E acetate and CBD. Lateral organization was also significantly affected as lipid domain size decreased and shape was altered. Conclusion: These initial results warrant further investigation to better understand the extent and mechanism behind a potential vitamin E acetate induced lung injury, as well as the combinatorial effects with cannabinoids.

\title{
Free radical scavenging potential and Antioxidant activity of SilverNanoparticle Coupled with Andrographis paniculata (AP-Ag NP) Against Carbon tetrachloride (CC14) induced toxicity in mice
}

\section{Soumendra Darbar, ${ }^{1}$ Srimoyee Saha, ${ }^{2}$}

${ }^{1}$ Head, Research \& Development Division, Dey's Medical, Kolkata, Kolkata, India

${ }^{2}$ Faculty Member \& Scientist, Jadavpur university, Kolkata, India

Purpose: Therapeutic exposure to Silver Nanoparticle Coupled with Andrographis paniculata (AP-AgNP) may lead to enhanced generation of free radicals and failure of endogenous antioxidant defense system ultimately resulting in oxidative stress. The present study aimed to evaluate the comparative efficacy of AP$\mathrm{Ag}$ NP against Carbon tetrachloride $\left(\mathrm{CCl}_{4}\right)$ induced oxidative stress. Methods: Male swiss albino mice were exposed to Carbon tetrachloride $\left(\mathrm{CCl}_{4}\right)$ for 28 days and were co-administered orally with AP-Ag NP $(50 \mathrm{mg} / \mathrm{kg}$ each) to evaluate effects on heme synthesis pathway, hepatic oxidative injuryand antioxidant potential. Results: Our study reported elevated ROS levels in carbon tetrachloride $\left(\mathrm{CCl}_{4}\right)$-treated group, suggesting free radical generation leading to oxidative stress conditions. Increased lipid peroxidation like elevated Thiobarbituric acid (TBARS) and reduced GSH, further signifies oxidative stress condition. Concomitant administration of Ag nanoparticle coupled with Andrographis paniculata led to pronounced recovery, suggesting it become a more effective scavenger of free radicals. We observed a significant elevation in TBARS level following $\mathrm{CCl}_{4}$ exposure and back to normal level after NP administration. Interestingly the level of plasma aspertate transaminase (AST), alanine transaminase (ALT), alkaline phosphatase (ALP) and gama glutamyl transaminase (GGT) the marker liver enzymes were restored to normal in the animals co-exposed to AP-Ag NP. On the other hand we observed a significant increase in hepatic SOD and Catalase activity on NP exposure which responded favourably to the co-administration of antioxidants. The results suggest a significantly elevated ROS, decreased blood and hepatic GSH levels, Superoxide dismutase and Catalase activities after Carbon tetrachloride $\left(\mathrm{CCl}_{4}\right)$ exposure. Co-administration of Nano-antioxidants (AP-Ag NP) increased SOD, CAT and GSH levels and was also beneficial in the recovery of oxidativeinjury and restoring inhibited aminolevulinate dehydratase (ALAD) activity. Conclusion: Our results suggest that Silver Nanoparticle Coupled with Andrographis paniculata (AP-Ag $\mathrm{NP})$ have the potential antioxidant effect in mice.

\section{0 years of Canadian innovations in life sciences and in health technologies}

\author{
Arielle Theoret, ${ }^{1}$ Michelle Savoie, ${ }^{2}$ Denis DeBlois, ${ }^{2}$ \\ ${ }^{1}$ Intern, ${ }^{2}$ Professor, Faculty of Pharmacy, University of Montreal, Canada
}

Purpose: The sector of life sciences and health technologies is rich in innovations that had a significative impact on human's health. However, the overall Canadian contribution in this field remains poorly known. To survey innovations that have emerged in the biopharmaceutical sector over the past 100 years in Canada. Methods: Herein, the term innovation was defined as a significative improvement in our capacity for therapeutic intervention in human health obtained by a process of biopharmaceutical research and 
development. As for our main inclusion criteria, the innovation had to be supported by a clinical or a technological proof of concept as recognized by independent observers (e.g. a regulatory agency, a thirdparty payer, or an investment firm). Innovations were classified as a) new molecule, b) repurposed molecule, c) diagnostic test, $d$ ) innovative formulation or e) novel production system. The innovation survey was conducted by querying government databases, regulatory agency websites and venture capital funds. In addition, interviews were carried out with stakeholders in the pharmaceutical field in academia or industry. Results: A total of 58 innovations met our inclusion criteria, covering all five categories. $78 \%$ of innovations were in the new molecule category. Notably, 6 of the 13 nanoformulations approved by a regulatory agency around the world were developed in Canada. The oldest recorded innovation was the discovery of insulin in the treatment of diabetes in 1921 and the most recent innovation was the discovery of Bamlanivimab, an antibody neutralizing SARS-Cov-2 approved by Health Canada on December 24 ${ }^{\text {th }}$ 2020, for the treatment of Covid-19. Data suggest that the rate of innovation is accelerating. Conclusion: The contributions of Canadian labs to the field of pharmaceutical innovation are significant, diverse, and growing, suggesting a potential for further global impact.

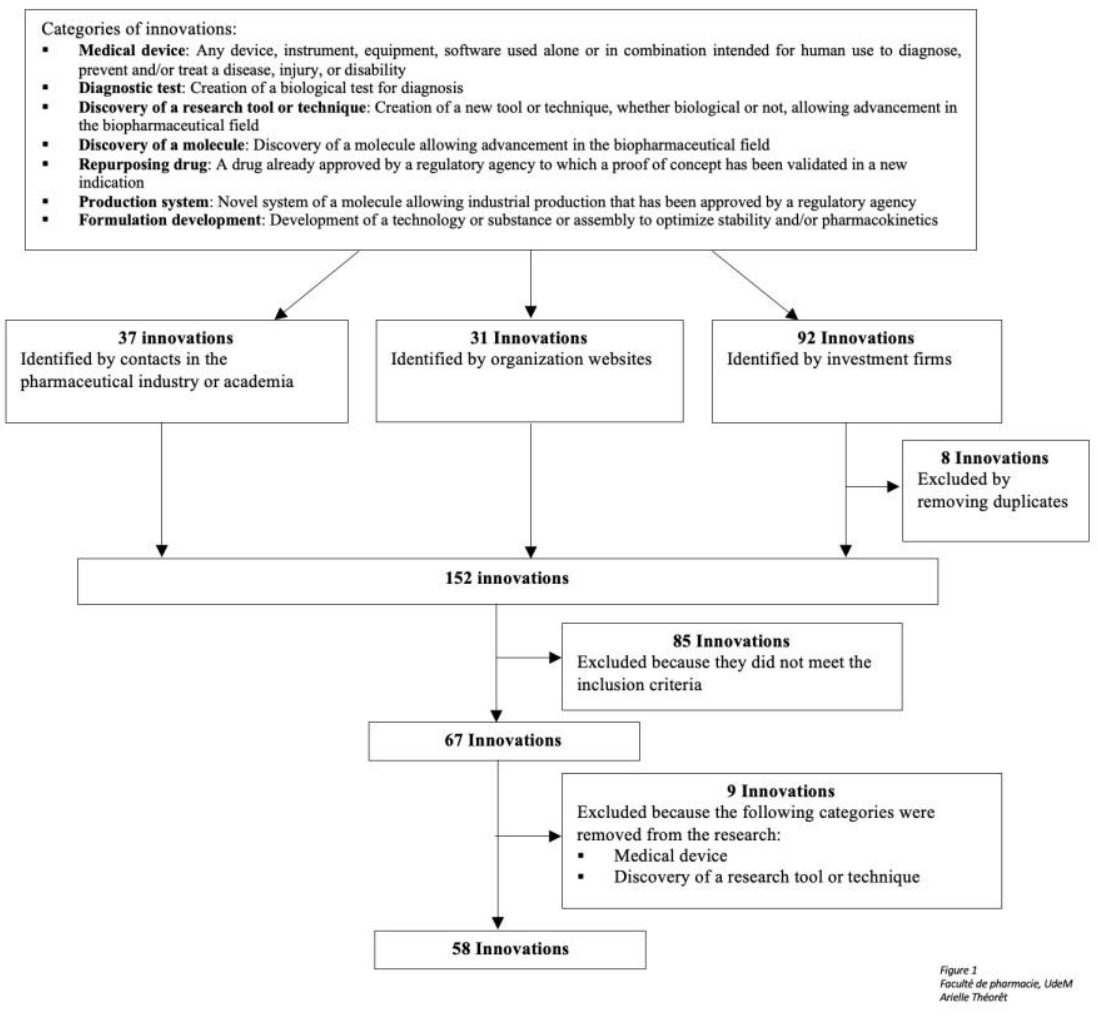

\title{
Phase IB double-blind randomized clinical study to evaluate the safety, tolerability and pharmacokinetics of a novel oral amphotericin b formulation (ICO-019) in healthy human subjects
}

\author{
Kishor Wasan, ${ }^{1}$ Ellen Wasan, ${ }^{2}$ Peter Hnik, ${ }^{3}$ \\ ${ }^{1}$ Distinguished University Scholar and Director of Research, University of British Columbia, Canada; \\ ${ }^{2}$ Assistant Professor, University of Saskatchewan, Canada; ${ }^{3}$ Chief Medical Officer, iCo Therapeutics Inc., \\ Canada
}

Purpose: The purpose of this double-blind randomized clinical study was to evaluate the safety, tolerability and pharmacokinetics of a novel oral amphotericin B capsule formulation (ICO-019) following multiple dose administration to health human subjects. Methods: Human subjects were dosed with either $100 \mathrm{mg}$ or 
$400 \mathrm{mg}$ of iCo-019 once daily for 10 consecutive days with an additional 10 days of follow-up (for a total of 20 days). This study was also placebo-controlled with one human subject placebo-controlled for each treatment group (100 and $400 \mathrm{mg}$ dose) was assessed. Amphotericin B plasma concentrations were determined by a validated assay using LC-MS. Pharmacokinetic noncompartmental analysis was performed using WinNonLin. Patients were monitored for gastrointestinal disturbances (i.e. nausea and diarrhea) and changes in kidney and liver function. Results: The primary endpoint of safety and tolerability of ICO-019 following oral administration of both doses were met including no signs of kidney, liver and gastrointestinal (GI) toxicities of note. ICO-019 at the $100 \mathrm{mg}$ dose achieved a median plasma Cmax of $26 \mathrm{ng} \mathrm{AmB} / \mathrm{mL}$ and AUC (0-inf) $991 \mathrm{hr} * \mathrm{ng} / \mathrm{mL}$ after day 1 of dosing and a median plasma Cmax of $44 \mathrm{ng} \mathrm{AmB} / \mathrm{mL}$ and AUC (0-inf) $1998 \mathrm{hr} * \mathrm{ng} / \mathrm{mL}$ after 10 day of dosing. This approximate doubling of the AUC (0-inf) measure between day 1 and day 10 was observed not only at the $100 \mathrm{mg}$ dose but at the $400 \mathrm{mg}$ dose as well (Table 1). Conclusions:: These data suggest that we have developed a novel oral amphotericin $B$ formulation that is safe and tolerable following multiple dosing to healthy human subjects. In addition, the prolonged plasma half-life and increased AUC observed in this human clinical study suggests that ICo-19 has a long circulation time which may result in the ability of the formulation to increase and sustain Amphotericin B concentrations within infected tissues without the associated GI, liver and kidney toxicity.

\begin{tabular}{|c|c|c|c|c|}
\hline & $\operatorname{Tmax}(\mathrm{hr})$ & $\mathrm{Cmax}$ (ng/ml) & $\begin{array}{l}\text { AUCO-inf } \\
(\mathrm{hr} * \mathrm{ng} / \mathrm{ml})\end{array}$ & T1/2 (hr) \\
\hline Day 1 & $9.6+/-8.1(n=5)$ & $25.7+/-4.6(n=5)$ & $\begin{array}{l}990.6+/-155.5 \\
(n=4)\end{array}$ & $24.5+1-4.9(n=4)$ \\
\hline Day 10 & $4.8+/-1.1(n=5)$ & $44.4+/-9.7(n=5)$ & $\begin{array}{l}1997.7+/-761.1 \\
(n=5)\end{array}$ & $31.0+/-8.8(n=5)$ \\
\hline
\end{tabular}

\section{Nano-delivery of a novel inhibitor of DNA repair for targeted sensitization of colorectal cancer to radiation induced DNA damage}

$\underline{\text { Sams Sadat }},{ }^{1}$ Melinda Wuest, ${ }^{2}$ Igor Paiva, ${ }^{3}$ Sirazum Munira, ${ }^{4}$ Nasim Sarrami, ${ }^{4}$ Forughalsadat Sanaee, ${ }^{3}$ Marco Paladino, ${ }^{5}$ Dennis Hall, ${ }^{6}$ Michael Weinfeld, ${ }^{7}$ Afsaneh Lavasanifar, ${ }^{8}$

${ }^{1} \mathrm{PhD}$ Candidate, Faculty of Pharmacy and Pharmaceutical Sciences, University of Alberta, Edmonton, AB, Canada, Edmonton, Canada; ${ }^{2}$ Research Associate, Department of Oncology, Faculty of Medicine and Dentistry, University of Alberta, Edmonton, AB, Canada, Edmonton, Canada; ${ }^{3}$ Postdoc, Faculty of Pharmacy and Pharmaceutical Sciences, University of Alberta, Edmonton, AB, Canada, Edmonton, Canada; ${ }^{4}$ MSc Student, Faculty of Pharmacy and Pharmaceutical Sciences, University of Alberta, Edmonton, AB, Canada, Edmonton, Canada; ${ }^{5}$ Postdoc, Department of Chemistry, Faculty of Science, University of Alberta, Edmonton, AB, Canada, Edmonton, Canada; 'Professor, Department of Chemistry, Faculty of Science, University of Alberta, Edmonton, AB, Canada, Edmonton, Canada; ${ }^{7}$ Professor, Department of Oncology, Faculty of Medicine and Dentistry, University of Alberta, Edmonton, AB, Canada, Edmonton, Canada; ${ }^{8}$ Professor, Faculty of Pharmacy and Pharmaceutical Sciences, University of Alberta, Edmonton, AB, Canada, Edmonton, Canada

Purpose: Our research team has developed a new potent lead inhibitor of a DNA repair enzyme, i.e., human polynucleotide kinase/phosphatase (PNKP), as a potential new drug for colorectal cancer (CRC). This PNKP inhibitor, i.e., A83B4C63, can sensitize CRC cells to DNA damaging therapeutics, but may act similarly on normal cells. Targeted delivery of A83B4C63 to tumor cells by nanoparticles (NPs) is expected to reduce its harmful effect on normal tissue particularly in combination with DNA damaging therapies. We investigated the radio-sensitizing activity of poly(ethylene oxide)-poly $(\alpha$-benzyl carboxylate- $\varepsilon$ - 
caprolactone) (PEO-b-PBCL) NPs of A83B4C63 (NP/A83) against HCT116 human CRC xenograft in mice. Methods: A83B4C63 was encapsulated in PEO-b-PBCL NPs or solubilized with the aid of Cremophor EL: Ethanol (CE/A83). The in vitro radio-sensitizing activity of CE/A83 and NP/A83 was investigated in HCT116 CRC cells by the evaluation of $\gamma$-H2AX protein overexpression. The biodistribution and radio-sensitizing activities of CE/A83 and NP/A83 intravenously administered at 25 $\mathrm{mg} / \mathrm{kg}$ (3 times a week) were determined in HCT116 CRC xenograft in mice. The fractionated radiation dose of $5 \mathrm{~Gy}, 24 \mathrm{~h}$ following intravenous administration of CE/A83 and NP/A83, was performed using Small Animal Radiation Research Platform (SARRP). Growth and proliferation of the tumors in xenograft mice were determined using IVIS bioluminescence imaging and positron emission tomography (PET). Results: Immunoblotting confirmed the significant and persistent induction of radio-sensitizing DNA damaging and apoptotic activities in CE/A83- and NP/A83-treated cells at low IR dose. No DNA damage and apoptotic activities were detected in CRC cells when solely treated with either CE/A83 or NP/A83. In both IVIS-imaging and PET scan, NP/A83-treated mice showed significantly higher growth retardation of tumor than CE/A83 when combined with radiation. This observation was attributed to the higher A83B4C63 levels in tumor xenografts of NP/A83-treated mice than CE/A83. Conclusion: The NP/A83 is a promising radio-sensitizer with preferential tumor accumulation in CRC.Acknowledgements: ACF, CIHR, NMIN.

\section{Evaluating the potential anti-viral activity of two polyene macrolide antibiotics against SARS-CoV-2}

\section{Kishor Wasan, ${ }^{1}$ Peter Hnik, ${ }^{2}$ Ellen Wasan, ${ }^{3}$ Volker Gerdts, ${ }^{4}$}

${ }^{1}$ Distinguished University Scholar and Director of Research, University of British Columbia, Canada: ${ }^{2}$ Chief Medical Officer, iCo Therapeutics Inc., Canada; ${ }^{3}$ Assistant Professor, University of Saskatchewan, Canada; ${ }^{4}$ CEO, VIDO-InterVac-University of Saskatchewan, Saskatoon, Canada

Purpose: The purpose of this study was to evaluate the potential anti-viral activity of two polyene macrolide antibiotics, amphotericin B (AmB) and nystatin (NYS) against SARS-CoV-2. Methods: Vero'76 kidney cells were seeded and grown overnight at $37^{\circ} \mathrm{C}$ in a $5 \% \mathrm{CO}_{2}$ environment to approximately $90 \%$ confluence in 96-well plates. The culture medium used was DMEM supplemented with 10\% FBS/PENSTREP. The virus SARS-CoV-2/Canada/ON/VIDO-01/2020/Vero'76/p.2 was diluted in DMEM supplemented with 2\% FBS/PEN-STREP to obtain a multiplicity of infection (MOI) of 0.1 (approximately $2000 \mathrm{TCID}_{50} /$ well). A serial dilution of AmB, NYS and Irbesartan (a drug known to bind to the ACE-2 receptor) were then added ( $\mathrm{n}=4$ at each concentration) to the infected cells. Medium alone was used as a control for virus replication, and untreated, uninfected control wells were also established. A separate plate was used to assess the cell toxicity of the test compounds by incubating uninfected cells with the serially diluted test compound solution. After 48 hours of incubation, cytotoxicity was measured by MTS assay. Cells were also examined for contamination at 24 hours, and for cytopathic effect (CPE) and cytotoxicity (if noticeable) at 48 hours. Viral titration by the TCID 50 assay of the supernatant was carried out by a serial 10-fold dilution. Cells were observed for $\mathrm{CPE}$ at 1, 3 and 5 days after the infection. Results: Amphotericin $\mathrm{B}(\mathrm{AmB})$ showed a significant reduction in the TCID50 titer, with the 50\% effective concentration (EC50) of $1.24 \mu \mathrm{M}$ (Figure 1). Nystatin (NYS), a compound similar in chemical structure to Amphotericin B and Irbesartan both exhibited substantially less active and would not be considered a suitable choice for further investigations. Conclusions:: Taken together these preliminary findings suggest that AmB may have significant anti-viral activity against SARS CoV-2. Further cell and animal studies are required to confirm these important preliminary findings. 


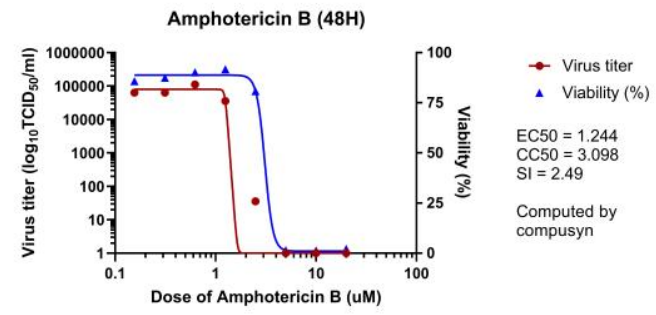

\section{Rational nanoparticle design for efficient transmucosal genome editing in lung epithelium} Belal Tafech, ${ }^{1}$ Jayesh A Kulkarni, ${ }^{2}$ Dominik Witzigmann, ${ }^{3}$ Pieter Cullis,${ }^{4}$ Sarah Hedtrich, ${ }^{5}$

${ }^{1} \mathrm{PhD}$ Student, Faculty of Pharmaceutical Sciences, University of British Columbia, Canada; ${ }^{2}$ Postdoctoral Fellow, Department of Biochemistry and Molecular Biology, University of British Columbia, Canada; ${ }^{3}$ Principal Research Fellow, Department of Biochemistry and Molecular Biology, University of British Columbia, Canada; ${ }^{4}$ Professor, Department of Biochemistry and Molecular Biology, University of British Columbia, Canada; ${ }^{5}$ Assistant Professor, Faculty of Pharmaceutical Sciences, The University of British Columbia, Vancouver, Canada

Purpose: Airway mucus protects the lung epithelium from foreign particles. However, its physicochemical properties also subdue delivery of promising therapeutics for pulmonary diseases. Cystic fibrosis (CF) is a life-shortening lung disease caused by mutations in the CFTR gene. Gene-editing technology CRISPR-Cas9 has great potential for curing such monogenic diseases. The challenge remains in efficiently delivering genetic cargo across the mucus. This study aims to identify nanoparticle (NP) characteristics for efficiently delivering CRISPR-Cas9 to the lung epithelium. Methods: Diffusion of NPs with different sizes and coated with hydroxyl, carboxyl, amine or Lipid-PEG (LNPs) was tested in mucus using Ibidi $\mu$-Slide chambers VI0.4/0.1. Next, LNPs entrapping CRISPR-CAS9 in the form of either mRNA or the ribonucleoprotein (RNP) complex were compared for their diffusivity in mucus. Furthermore, the transfection, gene-editing efficiency and biocompatibility of LNPs was examined in primary human lung epithelial cells in vitro. Results: Our results showed that LNPs $(\sim 120 \mathrm{~nm})$ diffused most efficiently in mucus compared to carboxylNPs (50nm) which showed 40\% less diffusivity. Amine and hydroxyl NPs (both 50nm) showed no significant diffusion. Furthermore, LNP-mRNA showed $\sim 2$-fold greater diffusion than LNP-RNP which is likely due to the smaller particle size compared to the RNP. Finally, LNP-mRNA transfected into lung epithelial cells showed less than $20 \%$ relative Indel percentages, significantly lower than compared to other epithelial cells such as skin cells. Conclusion: LNP showed the highest diffusion in lung mucus, likely due to PEG combining both amphiphilic nature and neutral charge thus minimizing LNP-mucus interaction. Furthermore, LNP encapsulating CRISPR-Cas9 mRNA rather than RNP showed superiority in lung mucus transport. However, LNPs tested showed insufficient uptake in epithelial lung cells which brings the need for further improvement in LNP design.

\section{Microfluidics production of polymeric microparticles with high drug loading of corticosteroids}

\section{Jack Bufton, ${ }^{1}$ Pauric Bannigan, ${ }^{2}$ Jeremy Pimental,${ }^{3}$ Christine Allen, ${ }^{4}$}

${ }^{1} \mathrm{PhD}$ Student, ${ }^{2}$ Post Doctoral Fellow, ${ }^{3}$ Lab Technician, University of Toronto, ${ }^{4}$ Professor, University of Toronto, Toronto, Canada

Purpose: Many techniques have been developed to produce polymeric microparticles (MPs); the most common are solvent-evaporation (SE) and spray-drying (SD) [1]. Microfluidics (MF) techniques are becoming increasingly popular in the pharmaceutical industry [2], as they provide good control over MP 
size and allow for continuous manufacturing. MF can also afford a higher encapsulation efficiency compared to SE and SD approaches [3]. In this study, we present a robust MF system, that can be assembled with minimal equipment, and is capable of continuous manufacturing of drug loaded poly(lactide-co-glycolide) (PLGA) MPs. To date, this system has afforded PLGA MPs containing the corticosteroids triamcinolone acetonide (TAA) and triamcinolone hexacetonide (TAH). Methods: A quartz flow-focusing MF chip and manifold were purchased from Dolomite Microfluidics. An HPLC pump provided flow for the continuous phase poly(vinyl alcohol), while a syringe pump provided flow of the dispersed phase (PLGA and drug in dichloromethane, at $25 \mathrm{wt} \%$ theoretical drug to material ratio). MP size distribution and the drug to material ratios were then evaluated using light microscopy and HPLC, respectively. Results: PLGA MPs loaded with either TAA or TAH had an average diameter of $\sim 50 \mu \mathrm{m}$. Average drug to material ratios were $14.5 \mathrm{wt} \%$, and $23.1 \mathrm{wt} \%$ for TAA and TAH loaded MPs, respectively. As a comparison TAA loaded MPs were also produced through the more conventional SE method, resulting in MPs with a higher degree of polydispersity. Preliminary in vitro drug release studies on the MPs formulations were also conducted. Conclusions: TAH and TAA were successfully loaded into PLGA MPs via continuous MF production. MPs prepared in this way were significantly more monodisperse than MPs manufactured via SE. Preliminary release results suggest that MF methods can result in less burst release at early timepoints. Continuous production of MPs in this manner has the potential to increase efficiency during manufacturing.

\section{Data-Driven Drug Formulation Design: Utilizing Machine Learning to Accelerate the Development of Long-Acting Injectable Drug Formulations}

\section{$\underline{\text { Pauric Bannigan }},{ }^{1}$ Zeqing Bao, ${ }^{2}$ Matteo Aldeghi,${ }^{3}$ Jeremy Pimental, ${ }^{4}$ Florian Hase,${ }^{3}$ Alan Aspuru-Guzik,${ }^{5}$ Christine Allen, ${ }^{6}$}

${ }^{1}$ Post Doctoral Fellow, ${ }^{2} \mathrm{PhD}$ student, ${ }^{3}$ Postdoctoral Research, ${ }^{4}$ Grad student, ${ }^{5}$ Professor, ${ }^{6}$ Professor, University of Toronto, Toronto, Canada

Purpose: Several types of long-acting injectable formulations (LAIFs) have been approved for human use, including drug-loaded polymeric microparticles (MPs) [1]. LAIFs offer several advantages over conventional medicines, including increased bioavailability, sustained drug levels, and improvements in patient compliance [2]. The first MP-based LAIF approved by the U.S. Food and Drug Administration, consists of leuprolide acetate encapsulated in poly(lactide-co-glycolide) (PLGA) microparticles (i.e., Lupron Depot ${ }^{\mathrm{TM}}$ ) [2], [3]. Despite the success of such PLGA-based systems, they cannot be universally employed to deliver all drugs. Each drug has its own unique physico-chemical properties and drug-material interactions have a significant influence on the performance of LAIFs. Machine learning (ML) offers the ability to predict how drug-material combinations will affect final formulation properties, such as release kinetics. The integration of ML into drug formulation development may not only reduce costs and accelerate the rate of MP-based LAIF development, but also lead to the discovery of new materials. Methods: 102 drug release profiles from both in-house experimental (47/102) and published studies (55/102) were used to create a database. Selected physicochemical features of these systems were used as inputs in ML models. Several ML models, including neural networks and mixture density networks, were trained and tested on this dataset using leave-one-group-out cross-validation. Results: For this relatively small dataset, drug release was predicted with a high degree of accuracy by deep learning models. The neural network models and mixture density networks described in this study achieved prediction accuracy, which was comparable to experimental uncertainty. Due to their architecture, the mixture density networks can also provide a measure of confidence in their predictions, Figure 1. Conclusions: The integration of ML models into drug formulation development may enable us to move away from the current trial-and-error approach and adopt a more efficient, cost effective data-driven formulation development process. 


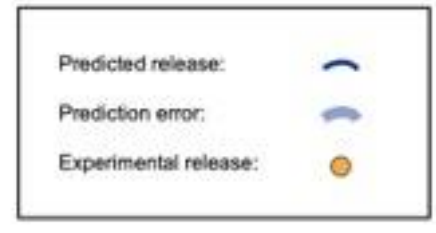

(a) paclitaxel leaded MP 1

(b) paclitaxel loaded MP 2

(c) triamcinolone acetonide loased MP 1

(c) triamcinolone acetonide loated MPP 2

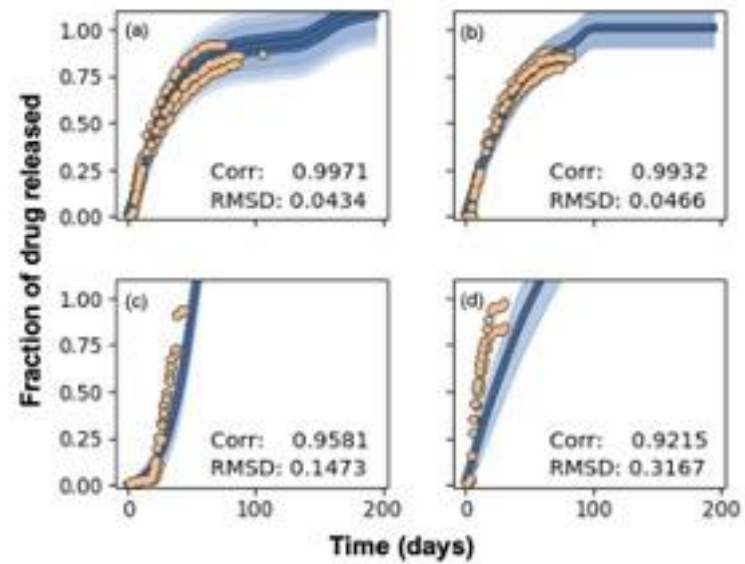

Time (days)

\section{Development of $\alpha \mathrm{v} \beta 6$-integrin targeted nanoparticles for delivery of PNKP inhibitors to non-small cell lung cancer (NSCLC) as monotherapy or combined with irinotecan}

Igor Paiva, ${ }^{1}$ Sams Sadat, ${ }^{2}$ Mohammad Vakili, ${ }^{3}$ Feridoun Karimi-Busheri, ${ }^{4}$ Marco Paladino, ${ }^{5}$ Dennis Hall, Michael Weinfeld, ${ }^{7}$ Afsaneh Lavasanifar, ${ }^{8}$

${ }^{1}$ Postdoc, Faculty of Pharmacy and Pharmaceutical Sciences, University of Alberta, Edmonton, AB, Canada, Edmonton, Canada; ${ }^{2} \mathrm{PhD}$ Candidate, Faculty of Pharmacy and Pharmaceutical Sciences, University of Alberta, Edmonton, AB, Canada, Edmonton, Canada; ${ }^{3}$ Research Associate, Faculty of Pharmacy and Pharmaceutical Sciences, University of Alberta, Edmonton, AB, Canada; ${ }^{4}$ Research Associate, Department of Oncology, Faculty of Medicine and Dentistry, University of Alberta, Edmonton, AB, Canada, Canada; ${ }^{5}$ Postdoc, Department of Chemistry, Faculty of Science, University of Alberta, Edmonton, AB, Canada, Edmonton, Canada; ${ }^{6}$ Professor, Department of Chemistry, Faculty of Science, University of Alberta, Edmonton, AB, Canada, Edmonton, Canada; ${ }^{7}$ Professor, Department of Oncology, Faculty of Medicine and Dentistry, University of Alberta, Edmonton, AB, Canada, Canada; ${ }^{8}$ Professor, Faculty of Pharmacy and Pharmaceutical Sciences, University of Alberta, Edmonton, AB, Canada, Edmonton, Canada

Purpose: The inhibition of polynucleotide kinase/phosphatase (PNKP), an enzyme involved in DNA repair, simultaneously with the disruption of phosphatase and tensin homologue (PTEN) has been shown to cause synthetic lethality in cancer cells. In non-small lung cancer (NSCLC), PTEN deficiency is seen in up to $70 \%$ of cases. Moreover, PNKP inhibition has been shown to act as a sensitizing agent for topoisomerase I inhibitors, such as irinotecan. PNKP is a ubiquitous enzyme, thus its targeted inhibition in cancer over normal cells is of immense importance. The aim of the present study was to develop NSCLC targeted nanoparticles encapsulating a relatively potent novel PNKP inhibitor, namely A83B4C63, and assess the anticancer activity of this formulation alone or in combination with irinotecan. Methods: Nanoparticles of poly(ethylene oxide)-poly(a-benzyl carboxylate-e-caprolactone) (PEO-PBCL) with maleimide group on their PEO segment were developed and used for conjugation of H2009.1 peptide with a terminal cysteine group. Cy5.5-labelled nanoparticles were used to evaluate cell uptake using flow cytometry. The cytotoxicity of A83B4C63 in NSCLC cell lines expressing different levels of PTEN expression with and without irinotecan treatment was assessed by MTS and colony-forming assays. Results: H2009.1-modified nanoparticles showed higher interaction with NSCLC cells overexpressing $\alpha_{\mathrm{v}} \beta_{6}$-integrin in comparison with their unmodified counterparts. The PTEN-positive NSCLC cells (i.e., H1975 cells) did not show loss of viability upon treatment with A83B4C63 monotherapy. The PTEN-deficient H1299 cells, on the other hand, showed less growth and colony formation following treatment with A83B4C63 monotherapy. 
Treatment with A83B4C63 made both cells, more sensitive to Irinotecan. The sensitization effect of A83B4C63 upon combination therapy with irinotecan was significantly enhanced for H1299 over H1975 cells. Conclusion: The results imply a potential for polymeric micellar formulations of A83B4C63 as mono-therapeutics in NSCLC cells with low PTEN expression and/or as targeted sensitizers to topoisomerase I inhibitors against NSCLC tumors.

\section{Clinical relevance: Why are enteric coatings failing in vivo?}

\section{Daniela Amaral Silva, ${ }^{1}$ Neal Davies, ${ }^{2}$ Michael Doschak,,${ }^{3}$ Jozef Al-Gousous, ${ }^{4}$ Nadia Bou-Chacra, ${ }^{5}$ Raimar Löbenberg, ${ }^{6}$}

${ }^{1} \mathrm{PhD}$ Candidate, ${ }^{2}$ Dean and Professor, ${ }^{3}$ Professor, Faculty of Pharmacy \& Pharmaceutical Sciences, University of Alberta, Edmonton, Canada; ${ }^{4}$ Adjunct Assistant Professor, Johannes Gutenberg-Universität Mainz, Canada; ${ }^{5}$ Professor, Universidade de Sao Paulo, Canada;

${ }^{6}$ Professor, University of Alberta, Edmonton, Canada

Purpose: Over the last 70 years several cases of in vivo failure of enteric coated (EC) formulations have been reported. The observed failures seem to be due to the slower than expected in vivo performance of EC products. Upon reaching the intestinal lumen, the dosage form is exposed to a bicarbonate buffered environment at much lower buffering capacity compared to those applied in compendial phosphate buffers. Hence, there is an urgent need to understand the behavior of EC products in bicarbonate buffer (BCB) and to revaluate the current dissolution methods used for such products. We hypothesized that the performance of EC products in BCB would be different compared with compendial phosphate buffer, giving more physiological insight with regards to the observed clinical failures. Methods: The current study mechanistically investigated the performance of five EC products available in the Canadian market. The evaluated parameters were the buffer system (bicarbonate buffer vs. phosphate buffer), buffer capacity, medium $\mathrm{pH}$ and formulations parameters (coat material and thickness). All dissolution tests were performed using an USP apparatus 2, $900 \mathrm{~mL}$ dissolution media, $75 \mathrm{rpm}$ rotation speed and temperature set at $37.0^{\circ} \mathrm{C}$. The coat thickness was assessed using Micro-CT scan. Results: All formulations displayed a fast release in phosphate buffer and complied with the compendial performance specifications. On the other hand, they all had a much slower drug release in bicarbonate buffer and failed the USP acceptance criteria. Also, the nature of the drug (acid vs base) impacted the dissolution behavior in BCB more than the coat thickness. Conclusion: This pilot study indicates that compendial dissolution test for enteric coated tablets lacks physiological relevance and it needs to be reconsidered. Bicarbonate buffer seems to be a clinically relevant dissolution medium for EC products. Thus, an in vivo relevant performance method for EC products is needed.
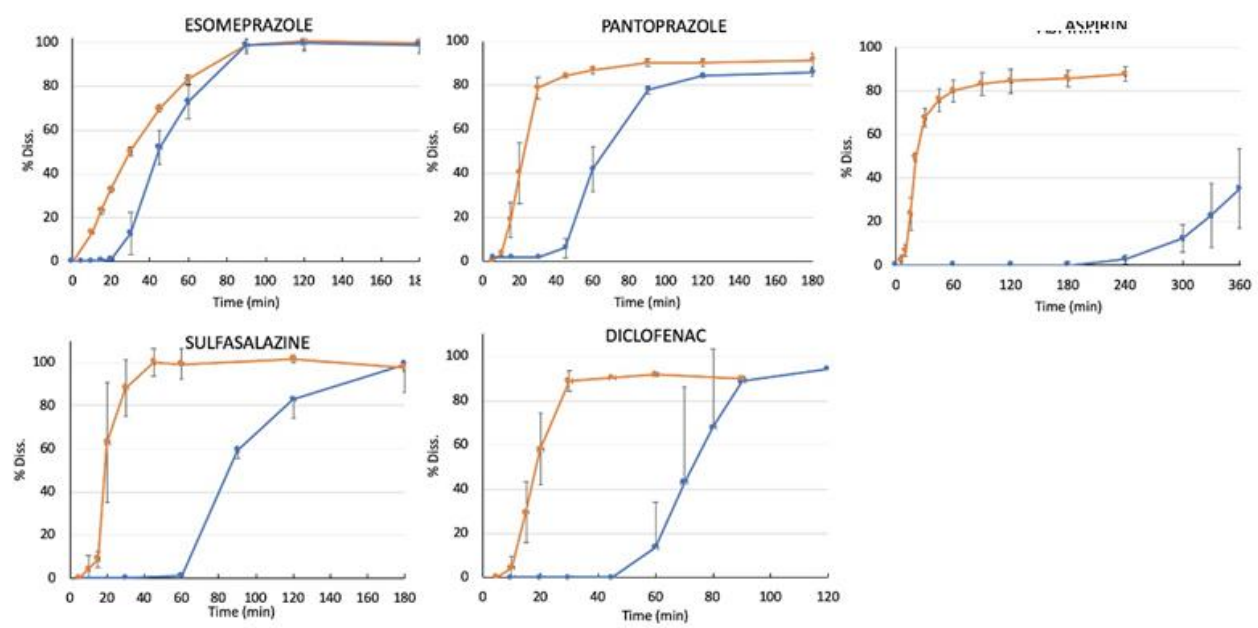


\section{Investigation of the release characteristics of Erzhi pills with its intended Traditional Chinese Medicine usage}

Jieyu Zuo, ${ }^{1}$ Chulhun Park, ${ }^{2}$ Michael Doschak,${ }^{3}$ Raimar Löbenberg, ${ }^{4}$

${ }^{1}$ Postdoctoral fellow, Faculty of Pharmacy \& Pharmaceutical Sciences, University of Alberta, Canada ${ }^{2}$ Postdoctoral fellow, Faculty of Pharmacy \& Pharmaceutical Sciences, University of Alberta, Edmonton, Canada

${ }^{3}$ Professor, Faculty of Pharmacy \& Pharmaceutical Sciences, University of Alberta, Edmonton, Canada ${ }^{4}$ Professor, University of Alberta, Edmonton, Canada

Purpose:s: Generally, Traditional Chinese Medicine (TCM) and its classic dosage form, pill, are both regarded as outdated and empirical systems. However, over the centuries, TCM has developed different types of pills to achieve various intended therapeutical purposes. This study selected one TCM herbal pill, Erzhi pill (EZP), which commonly applies in the long-term clinical management of post-menopausal symptoms to investigate if the pharmaceutical release mechanism of EZP is in alignment with its intended TCM usage. Methods: The release of specnuezhenide from three EZPs (self-made, Leiyunshang, and Renhe) in simulated gastric fluid (SGF), acetate buffer ( $\mathrm{pH} 4.5$ buffer), and simulated intestinal fluid (SIF) were investigated in dissolution testing for $4 \mathrm{~h}$. The water uptake capacity and erosion extent of the three EZPs were investigated using swelling and erosion studies. The drug release mechanism was further assessed through the statistical model fitting, using DDSolver software. Results: The release of specnuezhenide from all three EZPs in SGF was less than 50\% within $4 \mathrm{~h}$. However, over $70 \%$ of the specnuezhenide was released from each EZP in both $\mathrm{pH} 4.5$ buffer and SIF. The swelling and erosion behaviors and the drug release mechanism of the three EZPs confirmed that the release rate from EZP followed a sustained release profile and it was an interactive combination of swelling and erosion. Conclusion: This study showed that TCM had not only theoretically considered sustained release from the pills but also formulated them to achieve the release pattern to manage chronic diseases. It also suggested that the consideration of drug release patterns can be traced back to before dissolution testing was invented. When establishing quality control standards for pills, the theoretical TCM usage and the actual release patterns need to be considered together. Funding: This study is supported by Mitacs, Canada (Mitacs Accelerate: IT13686) and JRC Pharmaceutics Inc.

\section{The Design Of Polymeric Microneedles For The Delivery Of Sensors For Real-Time Physiological Monitoring}

Samuel Babity, ${ }^{1}$ Davide Brambilla, ${ }^{2}$

${ }^{1} \mathrm{PhD}$ Student, ${ }^{2}$ Assistant Professor, University of Montreal, Canada

Purpose: In addition to their well-known applications in the delivery of vaccines and therapeutics, microneedles present an excellent platform for assessing human health through the skin. Indeed, the skin the largest organ in the human body - carries a great deal of health-related information. Specifically, the dermal interstitial fluid (ISF) has been found to contain biological analytes (i.e. ions, small molecules, and proteins) in similar concentrations to the blood. [1] Due to their intrinsic features, microneedles can serve as useful tools to help access these analytes.By delivering rationally designed fluorescent sensors using dissolving polymeric microneedles, it is possible to generate a functional fluorescent medical tattoo, capable of sensing and monitoring biological analytes of interest (Figure 1). Previous works have used similar fluorescent medical tattoos for the structural and functional imaging of dermal lymphatic vessels. [2] This non-invasive functional tattoo could be further extended to the monitoring of other physiological parameters. Methods: Fluorescent sensors for biological analytes were synthesized and validated to ensure compatibility, stability, sensitivity, and specificity in the skin. Further, microneedles containing the sensors 
were made, and the resulting microneedle tattoos were delivered in vitro or in vivo, where their performance was assessed through fluorescence microscopy. Results: As a proof-of-concept, ROS-sensing dyes were synthesized and modified, with results indicating suitability for use in the skin. Further, the sensors were successfully loaded into microneedles, and their performance was evaluated using ex vivo skin models, human-based inflammatory skin models, and in vivo rat models. Conclusions: Dissolving polymeric microneedles appear suitable for delivering a fluorescent sensor to the skin, and this technology displays potential for sensing a wide variety of analytes through the skin, with implications for non-invasive at-home or point-of-care monitoring and diagnostics. Coupled with a portable fluorescence detector, as has been previously described [2], this technology could ultimately greatly improve the state of non-invasive personalized healthcare monitoring.

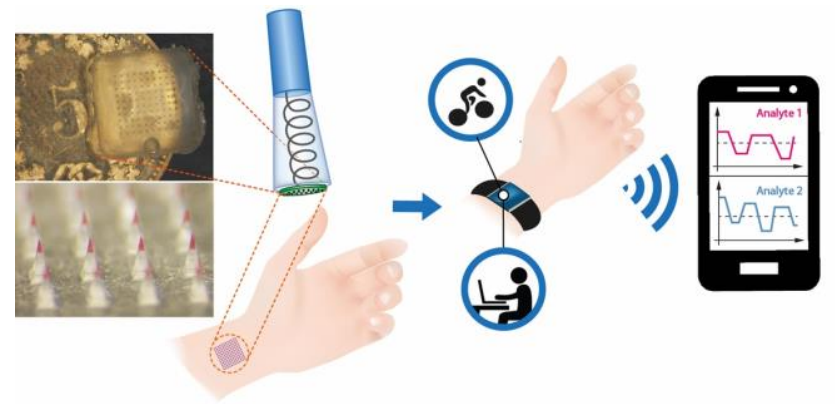

\title{
Affinity-based dextran macroporous scaffolds for the controlled presentation and supply of biomacromolecules for drug release and tissue engineering applications
}

\author{
$\underline{\text { Romane Oliverio }},{ }^{1}$ Gregory De Crescenzo, ${ }^{2}$ Xavier Banquy, ${ }^{3}$ \\ ${ }^{1} \mathrm{PhD}$ Candidate, Polytechnique Montréal, Institute of Biomedical Engineering, Canada \\ ${ }^{2}$ Polytechnique Montréal, Department of chemical engineering, Canada \\ ${ }^{3}$ Université de Montréal, Faculty oh pharmacy, Canada
}

Purpose: Dextran, a low-fouling, easily functionalized and biocompatible polysaccharide, is a promising candidate for drug release or tissue engineering applications. In this study, we present an affinity-based strategy to biofunctionalize macroporous dextran scaffolds with proteins and nanoparticles. The proposed platform is based on an heterodimeric coiled-coil, formed of the two peptides E-coil and K-coil. K-coil is covalently grafted to the dextran scaffold, while proteins of interests or nanoparticles are tagged with an Ecoil peptide. Besides, we propose a novel approach to quantify the amount of proteins grafted on nanoparticles, which allows us to rationally design our platform based on the valency of the E-coil/K-coil interaction (1). Methods: Dextran hydrogels are chemically cross-linked on macroporous polylactic acid molds to create an interconnected pore network. K-coil grafting to dextran hydrogels is achieved by click chemistry. The amount of proteins per nanoparticle (polymeric, metallic or inorganic) is determined using an amine-reactive reagent, after protein hydrolysis and nanoparticle degradation. Results: E-coil-tagged monomeric proteins are efficiently loaded into K-coil-functionalized gels (partition coefficient: 5.5). Over 10 days, $44 \%$ of the loaded proteins are released. K-coil-functionalized hydrogels are efficiently loaded with dimeric E-coil-tagged proteins (coefficient partition: 15). Over 10 days, $12 \%$ of the loaded proteins are released, suggesting a strong affinity effect. Our strategy to quantify the valency of the functionalized nanoparticles allow to detect as few as two proteins per nanoparticle (1). This feature allows precise control of the platform. Conclusions: These results demonstrate that the E-coil/K-coil affinity system is a good candidate for the efficient capture of biomolecules and nanoparticles within the scaffolds. Besides, the strength of the E-coil/K-coil interaction can be easily tuned, thus allowing to modulate the release profiles. These parameters, alongside with the macroporosity of the platform, make those hydrogels ideal candidates for both drug release and tissue engineering applications. 


\section{Tunable mucoadhesion and mucopenetration using self-assembled brush-block copolymer-based nanoparticles.}

Ridhdhi Dave, ${ }^{1}$ Andrew Singh, ${ }^{1}$ Lili Zhang, ${ }^{2}$ Maya Ziolkowska, ${ }^{3}$ Cecile Fradin, ${ }^{4}$ Heather Sheardown,${ }^{5}$ Todd Hoare, ${ }^{5}$

${ }^{1} \mathrm{PhD}$ Student, McMaster University, Department of Chemical Engineering, Canada

${ }^{2} \mathrm{PhD}$ Student, McMaster University, Department of Physics, Canada

${ }^{3}$ Undergraduate Student, McMaster University, Canada

${ }^{4}$ Professor - Department of Physics and Astronomy, McMaster University, Department of Physics, Canada

${ }^{5}$ Professor - Department of Chemical Engineering, McMaster University, Department of Chemical

Engineering, Canada

Purpose: Self-assembled nanoparticles (NPs) are promising drug delivery vehicles for the eye. Current methods often use block copolymers based on poly(ethylene glycol) (PEG); however, PEG offers limited chemical functionality and control over mucosal adhesion/penetration ${ }^{1,2}$. Herein, we created self-assembled NPs from a block copolymer of poly(oligo (ethylene glycol) methacrylate) (POEGMA), a brush polymer derivative of PEG, and poly(lactic acid) (PLA) that offers easy functionalization (via free radical copolymerization) while facilitating tunable molecular weights ${ }^{3}$. We hypothesize that controlling the PEG sidechain length in the POEGMA brush can control mucopenetration/mucoadhesion. Methods: NPs with tunable mucoadhesive/mucopenetrative properties were prepared through self-assembly of PLA and POEGMA-based amphiphilic block copolymers. POEGMA ${ }_{n}$ blocks were polymerized to different chain lengths using OEGMA monomers $(\mathrm{n}=2-40)$. NPs were self-assembled using flash nanoprecipitation and characterized for size (DLS) and morphology (TEM). POEGMA blocks were functionalized with amine functional groups to incorporate fluorescent dyes (i.e., Cy5) to enable particle tracking. Mucoadhesion was assessed via rheological synergism experiments with native mucin $(10 \mathrm{w} / \mathrm{w} \%)$. Mucopenetration is assessed via FCS wherein the diffusion coefficients of NPs were measured in mucin solutions $(5 \mathrm{mg} / \mathrm{mL})$. _ Results: Small $(<250 \mathrm{~nm})$ and monodisperse (PD< 0.2) nanocarriers were synthesized, with the size of the hydrophilic block influencing both the NP size (Figure 1A) and mucoadhesion/ mucopenetration (Figures 1B-E). NPs fabricated from long side chain amphiphilic block copolymers (PLA-POEGMA ${ }_{n=20,40}$ ) demonstrated mucoadhesion through an increase in storage ( $\left.G^{\prime}\right)$ moduli when mixed with native mucin; in comparison short chain counterparts (PLA-POEGMA $A_{n=2,7-9}$ ) showed no significant mucoadhesion. Fluorescently labeled amphiphilic block copolymers of PLA-POEGMA $\mathrm{n}_{\mathrm{n}} \mathrm{Cy} 5$ were subsequently prepared to enable FCS measurements for assessing mucopenetration, the results of which will be presented. Conclusion: NPs self-assembled from PLA-POEGMA block copolymers can mimic the favorable properties of PLGA-PEG regarding balancing mucopenetration and mucoadhesion (key to accessing the eye), while introducing chemical versatility through functionalization via simple free radical copolymerization of functional comonomers. 


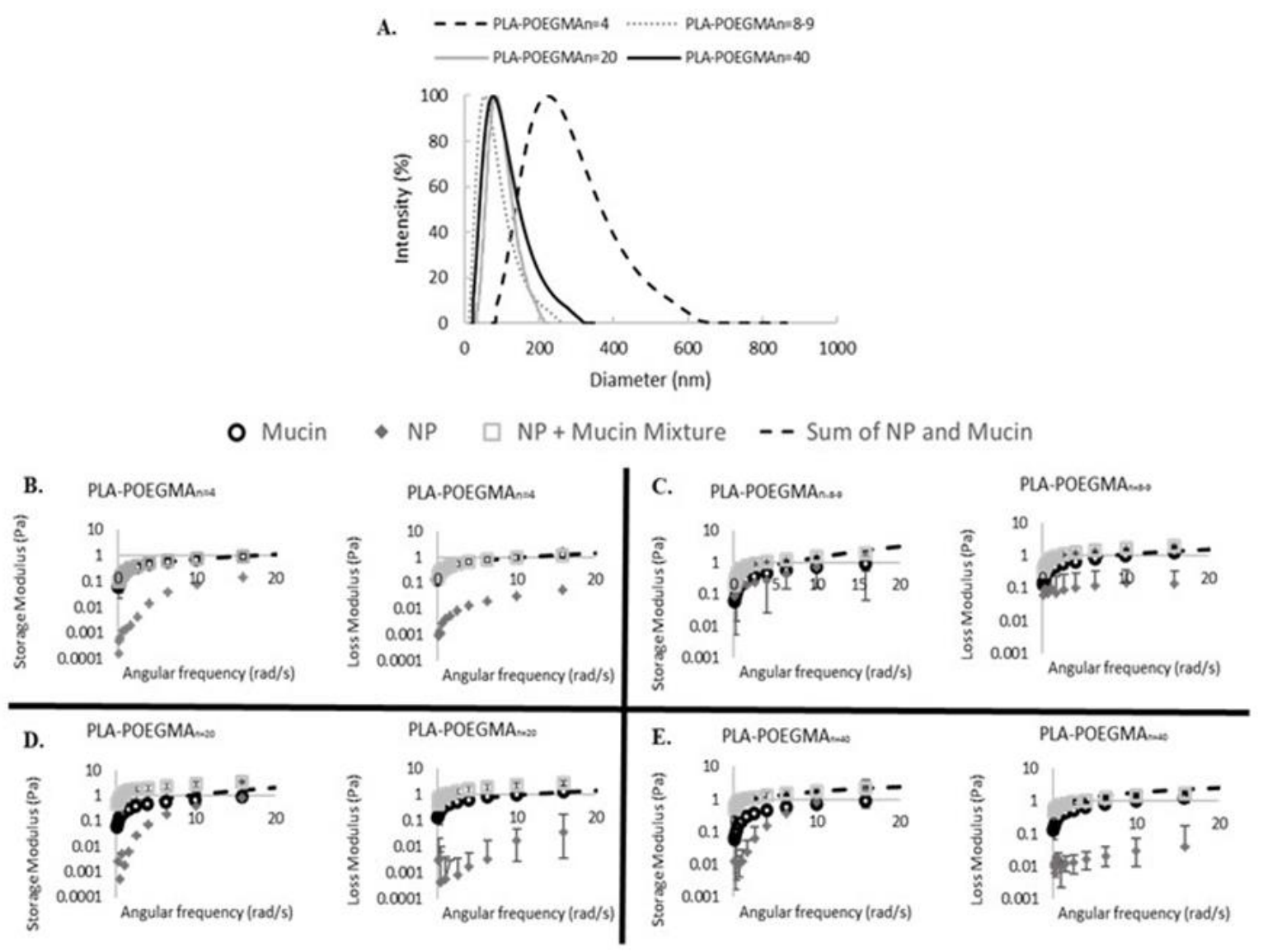

\section{Protecting Group-Minimized Peptide Synthesis Using Peptide Thioacid}

Kazuki. Oikawa, ${ }^{1}$ Toshifumi. Tatsumi, ${ }^{2}$ Koki. Sasamoto, ${ }^{1}$ Ryo. Hirano, ${ }^{1}$ Takuya. Matsumoto,${ }^{3}$ Kounosuke. Oisaki, ${ }^{4}$ Motomu. Kanai, ${ }^{5}$

${ }^{1}$ Graduate student, University of Tokyo, Japan

${ }^{2}$ Postdoctoral Fellow, University of Tokyo, Japan

${ }^{3}$ Assistant professor, Kanazawa University, Japan

${ }^{4}$ Lecturer, University of Tokyo, Japan

${ }^{5}$ Professor, University of Tokyo, Japan

Purpose: Peptides are attractive therapeutic modality taking advantages of both small molecules and biologics. A liquid-phase synthesis of peptides can broadly introduce natural and non-natural amino acids and is suitable for mass production. However, conventional peptide synthesis, elongating the peptide chain from the $\mathrm{C}$-terminus to the $\mathrm{N}$-terminus, generates a large amount of wastes derived from the $\mathrm{N}$-terminus protecting groups and condensation reagents, thereby increasing environmental burden and manufacturing cost. Therefore, we aimed to establish an $\mathrm{N}$-terminus-to-C-terminus peptide elongation method that minimizes wastes by using main chain-unprotected amino acids as starting materials. Methods: We previously reported a catalytic conversion of peptide $\mathrm{C}$-terminus carboxylic acids to peptide thiocarboxylic acid (1), which can be regarded as an activated acyl donor after aerobic dimerization to diacyl disulfide intermediates. Based on the method, we studied the coupling reaction between peptide thiocarboxylic acids 
and main chain-unprotected amino acids. Results: Use of a nucleophilic additive greatly suppressed the problematic epimerization. The optimized protocol of peptide coupling was applicable to various amino acids, including those with great steric hindrance, and also to iterative and convergent peptide coupling. Conclusion: We established a liquid-phase peptide synthesis that reduces the use of protecting groups. This method has potential to achieve a large-scale iterative and convergent peptide synthesis.

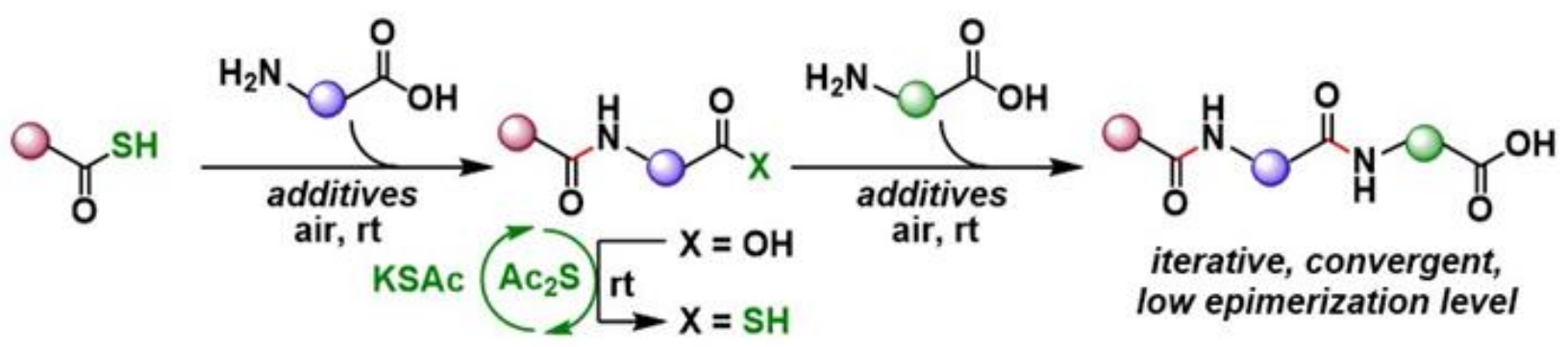

\section{Design and development of a novel Andrographolide formulation for intra-articular drug delivery systems}

Thitianan. Kulsirirat, ${ }^{1}$ Korbtham. Sathirakul, ${ }^{2}$ Noriyasu. Kamei, ${ }^{3}$ Mariko. Takeda-Morishita, ${ }^{4}$

${ }^{1}$ Post-doctoral, Department of Pharmacy, Faculty of Pharmacy, Mahidol University, Bangkok 10400, Bangkok, Thailand

${ }^{2}$ Professor, Department of Pharmacy, Faculty of Pharmacy, Mahidol University, Bangkok 10400, Bangkok, Thailand; ${ }^{3}$ Professor, Laboratory of Drug delivery systems, Faculty of Pharmaceutical Sciences, Kobe Gakuin University, Kobe, Japan; ${ }^{4}$ Professor, Laboratory of Drug delivery systems, Faculty of Pharmaceutical Sciences, Kobe Gakuin University, Kobe, Japan

Purpose: Andrographolide (AG), Thai herbal extract, is widely used for treatment of many chronic diseases and well-known traditional medicinal compound in Southeast Asia. Interestingly, AG has been reported to have inhibitory effects on osteoclast function and anti-inflammatory properties. This study aimed to develop and optimize the formulation of AG loaded poly (lactic-co-glycolic acid) (PLGA) nanocarriers and gelatinbased hydrogel to prolong the retention time in the joint. Methods: Two types of AG loaded acid and ester terminated end group PLGA nanoparticles were prepared by emulsion solvent evaporation method and further embedded into gelatin-based hydrogel (sheet and beads types). The in vitro release of AG from the formulations were determined by dialysis membrane method up to 35 days. Furthermore, In the in vivo retention time study, the 1,1'-dioctadecyltetramethyl indotricarbocyanine iodide (DiR) fluorescence dye was used to represent AG in the formulations. Fluorescent images and amount of DiR remaining at the site of implantation and injection were determined by IVIS imaging (day 1 until weeks 8). Results: The AG loaded ester terminated end group PLGA polymer gradually released AG from the PLGA nanoparticles when compared with AG solution. Importantly, the combined use of gelatin-based hydrogel with the AG loaded PLGA nanoparticles significantly delayed the AG release more than 1 month. The IVIS images showed that hydrogel beads displayed a significantly brighter and greater fluorescent intensity of DiR than hydrogel sheet whereas the DiR solution is rapidly cleared from joint. Conclusion: The results successfully displayed a long-term sustained release almost 2 months for hydrogel sheet and more than 2 months for hydrogel beads. Therefore, these formulations can provide a novel strategy for the local management of osteoarthritis disease. 


\title{
Development of allergic rhinitis immunotherapy using antigen-loaded multifunctional small extracellular vesicles
}

\author{
Wen liu, ${ }^{1}$ Maki Ota, ${ }^{2}$ Yuki Takahashi, ${ }^{3}$ Yoshinobu Takakura, ${ }^{4}$ \\ ${ }^{1}$ D3, Graduate School of Pharmaceutical Sciences, Kyoto University, Canada \\ ${ }^{2}$ B5, Graduate School of Pharmaceutical Sciences, Kyoto University, Canada \\ ${ }^{3}$ associate professor, Graduate School of Pharmaceutical Sciences, Kyoto University, Canada \\ ${ }^{4}$ professor, Graduate School of Pharmaceutical Sciences, Kyoto University, Canada
}

Purpose: Allergic rhinitis is caused by breakdown of Th1/Th2 balance in which allergen-induced Th2 immune response predominates over Th1 immune response resulting in IgE-mediated anaphylaxis. Herein, we used small extracellular vesicles ( $\mathrm{sEV}$ ) that are cell-derived membrane vesicles with a particle size of $100 \mathrm{~nm}$ as simultaneous delivery carrier for allergen (ovalbumin, OVA) and CpG DNA, which is an adjuvant that can induce Th1 immune response, for the treatment of allergic rhinitis. Methods lactadherin (LA) and group-specific antigen (Gag) are sEV-tropic proteins that can be located to the outer surface and inner surface of sEV, respectively. Plasmid DNA expressing the fusion protein of streptavidin (SAV; a protein that binds to biotin with high affinity) and LA (SAV-LA) and plasmid DNA expressing the fusion protein of Gag-OVA were simultaneously transfected to the Human Embryonic Kidney 293 (HEK293) cells. sEV were collected from the transfected HEK293 cells by ultracentrifugation and subsequently reacted with biotinylated CpG DNA to prepare CpG DNA modified OVA loaded sEV (CpG-OVA-sEV). Allergic rhinitis model mice were treated by intranasal administration of sEVs. Results: CpG DNA and OVA were successfully loaded to the outer surface and inner surface of sEV, respectively. CpG-OVA-sEV possessed an average particle size of $90 \mathrm{~nm}$ and an average zeta potential of $-30 \mathrm{mV}$. CpG DNA modification showed no influence on uptake of sEV by dendritic cells and CpG-OVA-sEV activated dendritic cells. Intranasal administration of CpG-OVA-sEV significantly enhanced OVA-specific IgG antibody titer, which suggests transformed Th1/2 balance. Moreover, allergic symptoms were alleviated by CpG-OVA-sEV administration compared with the control group. In addition, IgE secretion in mouse serum was also significantly reduced. Conclusion: It was shown that the $\mathrm{CpG}-\mathrm{OVA}$-sEV can be a useful therapeutic method for the treatment of allergic rhinitis.

\section{Development of a small extracellular vesicle (sEV) labeling method utilizing fusion protein of fluorescent proteins and SEV-tropic protein for the detection of phosphatidylserine-status of sEVs}

Yuki Kobayashi,,${ }^{1}$ Yuki Takahashi, ${ }^{2}$ Yoshinobu Takakura, ${ }^{3}$

${ }^{1}$ Graduated school student, Graduate School of Pharmaceutical Sciences, Kyoto University Department of Biopharmaceutics and Drug Metabolism, Canada

${ }^{2}$ associate professor, Graduate School of Pharmaceutical Sciences, Kyoto University, Canada

${ }^{3}$ professor, Graduate School of Pharmaceutical Sciences, Kyoto University, Canada

Purpose: Small extracellular vesicles (sEVs) are membranous vesicles secreted by cells with a diameter of approximately $100 \mathrm{~nm}$ and are expected to be applied as a modality for novel drug delivery systems. It has been reported that $\mathrm{sEVs}$ are a heterogeneous population and that $\mathrm{sEVs}$ with different physicochemical properties can exhibit different behaviors after administration into live animals. We have previously found that phosphatidylserine (PS) content in sEVs as an important factor that can affect behavior of sEVs. Therefore, in the present study, we developed method to label sEVs by using two types of fusion proteins composed of sEVs-tropic proteins and fluorescent proteins: one is a fusion protein composed of lysosome- 
associated membrane protein 2 (Lamp2; sEV-tropic protein) and mCherry (mCherry-Lamp2) to detect bulk sEVs, and the other is a fusion protein composed of lactadherin (LA; PS-binding protein) and GFP (GFPLA) to detect PS-positive sEVs. Methods: sEVs were collected from the culture supernatant of B16BL6 cells double-transfected with mCherry-Lamp2 and GFP-LA by sequential centrifugation, followed by 0.2 $\mu \mathrm{m}$ filtration, and ultracentrifugation. Fluorescence derived from sEVs was observed by fluorescence microscopy. The morphology of sEV was confirmed by transmission electron microscopy (TEM). Results: Fluorescence derived from mCherry-Lamp2 as well as GFP-LA was observed in the collected sEVs by Fluorescence microscope. The morphology of sEV collected from the transfected cells observed by TEM was comparable to control sEV, and their size was approximately $100 \mathrm{~nm}$. Conclusion: We succeeded in the development of a sEV labeling method utilizing fusion proteins. The developed can be useful method to detect the amount of PS in the collected sEVs.

\title{
Effect of water on the stability of Carbamazepine/Polyvinylpyrrolidone solid dispersions: Combined Molecular Dynamics and Fragment Molecular Orbital methods
}

\author{
Hugo Sato, ${ }^{1}$ Xiaohan Ma, ${ }^{2}$ Yusuke Kawashima, ${ }^{3}$ Koji Okuwaki, ${ }^{4}$ Takayuki Furuishi, ${ }^{3}$ Kenjirou Higashi, \\ Kaori Fukuzawa, ${ }^{6}$ Etsuo Yonemochi, ${ }^{7}$ \\ ${ }^{1}$ Ph.D. candidate, School of Pharmacy and Pharmaceutical Science, Hoshi University, Canada \\ ${ }^{2} \mathrm{Ph} . \mathrm{D}$. candidate, Graduate School of Pharmaceutical Science, Chiba University, Canada \\ ${ }^{3}$ Assistant professor, School of Pharmacy and Pharmaceutical Science, Hoshi University, Canada \\ ${ }^{4}$ Assistant professor, Faculty of Science, Rikkyo University, Canada \\ ${ }^{5}$ Associate professor, Graduate School of Pharmaceutical Science, Chiba University, Canada \\ ${ }^{6}$ Associate professor, School of Pharmacy and Pharmaceutical Science, Hoshi University, Canada \\ ${ }^{7}$ Full professor, School of Pharmacy and Pharmaceutical Science, Hoshi University, Canada
}

Purpose: It is known that amorphous Solid dispersion (ASD) has reduced amorphous stability under high humidity. In this study, we investigate how the addition water to ASD consisting of Carbamazepine (CBZ) and Polyvinylpyrrolidone (PVP) affects the API-additive interactions at nano level by using computational chemistry. [Method] ASD molecular structure was created by molecular dynamics simulation. The melt quenching was carried out for the initial structure with randomly placed CBZ, PVP and water molecules in the ASD. For resulting structures, Fragment Molecular Orbital (FMO) calculation was performed to analyze the detailed interaction energy. Results: By adding 10\% of water to the ASD consisting of CBZ: PVP $=3: 7$ (weight ratio), the glass transition predicted from the volume fluctuation in the cooling process was significantly reduced after adding water. In addition, the number of hydrogen bonds between the CBZ amide group and the PVP carbonyl group, which contribute to stability, was reduced by about $56 \%$ compared to the without water system. As a result of FMO calculation, although the interaction energy between CBZ and CBZ slightly change, the interaction energy between CBZ and PVP decreased by about $30 \%$ in the presence or absence of water. Conclusion: By using MD and FMO calculation, we can be predicted that the presence of water reduces the hydrogen bonds between CBZ and PVP and the stability. These methods are useful to evaluate detailed amorphous molecular structures and weak interactions that are difficult to evaluate experimentally. This study shows that MD and FMO are expected to be used to develop efficient and high-quality formulations. 


\title{
Efficient cancer-targeted drug delivery using anti-cancer drug-modified mesenchymal stem cells
}

\author{
Yukiya Takayama, ${ }^{1}$ Kosuke Kusamori, ${ }^{2}$ Ikumi Kiyama, ${ }^{3}$ Makiya Nishikawa, \\ ${ }^{1}$ Postdoctoral fellow, Laboratory of Biopharmaceutics, Faculty of Pharmaceutical Sciences, Tokyo \\ University of Science, Canada \\ ${ }^{2}$ Assistant professor, Laboratory of Biopharmaceutics, Faculty of Pharmaceutical Sciences, Tokyo \\ University of Science, Canada \\ ${ }^{3}$ Master's course student, Laboratory of Biopharmaceutics, Faculty of Pharmaceutical Sciences, Tokyo \\ University of Science, Canada \\ ${ }^{4}$ Professor, Laboratory of Biopharmaceutics, Faculty of Pharmaceutical Sciences, Tokyo University of \\ Science, Canada
}

Purpose: Mesenchymal stem cells (MSCs) are reported to accumulate to tumors after systemic injection. Thus, the cells can be useful as carriers for anti-cancer drugs. To achieve this, it is expected to modify MSCs with a large amount of anti-cancer drugs without any significant changes of their characteristics. In this study, we attempted to modify the cell surface of MSCs with doxorubicin-loaded liposomes (DOX-Lip) using the avidin-biotin complex (ABC) method and evaluated the anti-cancer effect of the DOX-Lipmodified MSCs. Methods: To modify murine mesenchymal stem cell line C3H10T1/2 cells with DOX-Lip, avidinated C3H10T1/2 cells were incubated with biotinylated DOX-Lip. The modification was evaluated using a confocal laser scanning microscope. The in vitro anti-cancer effect of DOX-Lip-modified MSCs was evaluated by co-culturing the MSCs with firefly luciferase-expressing murine colon adenocarcinoma colon26/fluc cells. The in vivo anti-cancer effect of DOX-Lip-modified MSCs was evaluated in colon26/fluc-bearing mice. Results: The fluorescence signal of DOX was detected on the surface of DOXLip-modified C3H10T1/2 cells. DOX-Lip modification hardly affected the viability and tumor tropism of C3H10T1/2 cells. The viability of colon26/fluc cells co-cultured with DOX-Lip-modified C3H10T1/2 cells remarkably decreased compared with that co-cultured with DOX-Lip. Furthermore, the fluorescent foci of DOX were detected inside green fluorescence protein-expressing colon26 (colon26/GFP) cells adjacent to the co-cultured DOX-Lip-modified C3H10T1/2 cells, indicating that DOX-Lip modified on the surface of C3H10T1/2 cells were directly delivered to colon26/GFP cells. Intravenously injected DOX-Lip-modified C3H10T1/2 cells remarkably suppressed the tumor growth in a lung metastasis mouse model. Conclusion: DOX-Lip modified on the surface of C3H10T1/2 cells by the ABC method were efficiently and directly delivered to colon 26 cells and suppressed the proliferation of colon 26 cells both in vitro and in vivo. These results indicate that anti-cancer drug modified MSCs can be useful for efficient cancer-targeted drug delivery.

\section{Evaluating the Therapeutic Efficacy and Biological Transport of Hydrophobized Poly(oligoethylene glycol methacrylate)-Based Nanogels for the Delivery of Lurasidone}

$\underline{\text { Madeline Simpson, }},{ }^{1}$ Brendan Fera, ${ }^{2}$ Jonathan Dorogin, ${ }^{3}$ Ashley Bernardo, ${ }^{4}$ Fahed Abu-Hijelh, ${ }^{5}$ Ram Mishra, ${ }^{6}$ Todd Hoare, ${ }^{7}$

${ }^{1}$ Postdoctoral Fellow, ${ }^{2}$ Master's Candidate, ${ }^{3}$ Research Associate, ${ }^{4} \mathrm{PhD},{ }^{5} \mathrm{PhD}$ Student, ${ }^{6}$ Professor, ${ }^{7}$ Professor, Department of Chemical Engineering, McMaster University, Hamilton, ON, Canaa

Purpose: Effective, long-term treatment of schizophrenia is challenging because of the many side effects associated with common antipsychotic drugs (APDs). Lurasidone (LUR) is an atypical APD with distinct pharmacological features that address the positive, negative and cognitive symptoms associated with schizophrenia. However, LUR has very limited aqueous solubility, leading to slow dissolution and poor 
absorption upon oral administration. Herein, we utilize nanogels, a class of soft, deformable nanocarriers, modified with hydrophobic domains via copolymerization of either butyl methacrylate (BMA) or methyl methacrylate (BMA) to promote LUR uptake and facilitate the intranasal (IN) delivery of the APD to improve its bioavailability. Methods: Nanogels are prepared by copolymerizing diethylene glycol methacrylate, acrylic acid (AA), BMA or MMA and ethylene glycol dimethacrylate (cross-linker). The nanogels were characterized using light scattering (size and mobility) and conductometric titration (functional group content). LUR was passively loaded into the nanogels, with excess drug removed using centrifugation. The pharmacodynamics of IN and intraperitoneally (IP) administered LUR-loaded nanogels were compared using a rodent model of schizophrenia (Figure 1). The in vivo biodistribution of fluorescently-labelled nanogels was assessed 3, 24 and 28 hours post-IN administration. Results: Up to $~ 3$ wt\% LUR loading can be achieved in nanogels prepared with 10 mol\% BMA while maintaining high cytocompatability with SH-SY5Y neuronal cells. IN administration of nanogels loaded with a low dose $(0.75 \mathrm{mg} / \mathrm{kg})$ of LUR achieve significant behavioural changes in the locomotor activity of MK-801-treated $(0.35 \mathrm{mg} / \mathrm{kg}$ ) rodents; analogous responses were only achieved with a $3 \mathrm{mg} / \mathrm{kg}$ dose of LUR when delivered IP. IN-administered nanogels remain localized in the nasal tissue for at least two days, with minimal accumulation in clearance organs. Conclusion: The reported hydrophobized nanogel drug delivery system has promising clinical potential as a minimally-invasive alternative for the delivery of hydrophobic APDs to the brain.

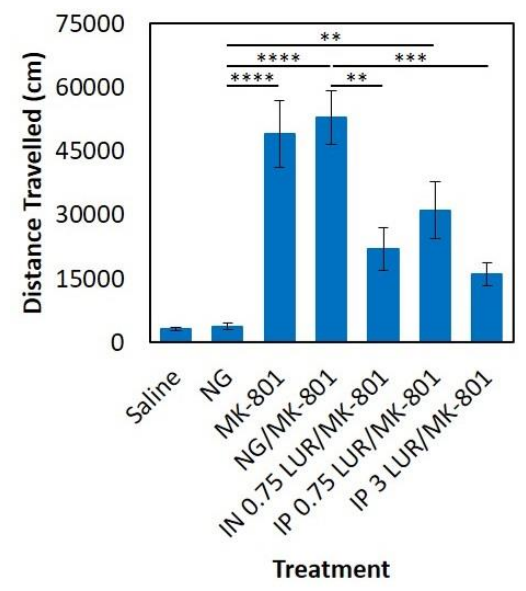

\title{
The Pharmaceutical Characterization and Analysis of commercially available Biological Hemp Products
}

\author{
Tyson Le, ${ }^{1}$ Rakesh Bhat, ${ }^{2}$ Shadab Alam, ${ }^{3}$ Michael Doschak, ${ }^{4}$ Neal Davies,${ }^{5}$ \\ ${ }^{1}$ Research Assistant, University of Alberta, Edmonton, Canada \\ ${ }^{2}$ Director, Laboratory Science, Applied Pharmaceutical Innovation (API), Edmonton, Canada \\ ${ }^{3}$ Industrial Postdoctoral Fellow, University of Alberta, Edmonton, Canada \\ ${ }^{4}$ Professor, Faculty of Pharmacy \& Pharmaceutical Sciences, University of Alberta, Edmonton, Canada \\ ${ }^{5}$ Dean and Professor, University of Alberta, Canada
}

Purpose: This study was done to develop and validate HPLC assays to quantify different cannabinoids i.e., Cannabigerol (CBG), Cannabidiol (CBD), Cannabidavarin (CBDV) and Tetrahydrocannabivarin (THCV) in commercially available hemp products of Blue Sky Biologicals. These compounds are major components found in commercial hemp products of unknown quantity. In this study, the characterization of Cannaflakes were done using microCT, scanning dynamic light scatter (DLS) and Transmission electron microscopy (TEM) techniques. Methods: Methanol was used to extract cannabinoids from commercial hemp products. 
Two different HPLC assay methods were developed for quantification. An isocratic mobile phase of acetonitrile:water $(75: 25)$ at a flow rate of $0.5 \mathrm{ml} / \mathrm{min}$ with the column oven temperature set at $30^{\circ} \mathrm{C}$ and the second method was developed using a gradient program $(0-27$ minutes $62 \% \mathrm{~B}, 27-55$ minutes $68 \%$ $\mathrm{B}, 55-62$ minutes $62 \% \mathrm{~B} . \mathrm{A}=$ Water and $\mathrm{B}=$ Methanol) with a flowrate of $1 \mathrm{ml} / \mathrm{min}$ and column oven temperature set at $50^{\circ} \mathrm{C}$. Both methods used a Luna ${ }^{\circledR} 5 \mu \mathrm{m} \mathrm{C} 18150 \times 4.6 \mathrm{~mm}$ column and detection used a wavelength of $210 \mathrm{~nm}$. DLS was used to characterize Cannaflake by determining the particle size and distribution by intensity in different dispersion mediums (anhydrous ethanol and methanol). Furthermore, morphology were determine using microCT scanning method. Results: Two HPLC methods were developed and validated to quantify cannabinoids. Standard curves display excellent linearity $\left(r^{2} \geq 0.99\right)$, from concentrations range between 1 to $50 \mu \mathrm{g} / \mathrm{mL}$. The intra-day and inter-day variation were negligible. microCT scans showed the distribution of the flakes. DLS data represent homogenously distributed particles in methanol as compared to anhydrous ethanol. Conclusions: Commercially available hemp products were quantified for their cannabinoids CBD, CBG, CBDV, and THCV using two HPLC methods developed. Furthermore, the product, Cannaflake organic extracts were characterized with DLS for particle size distribution.

\title{
Investigation of the Chemotherapeutic Potential of Cannabinoids in Vitro with Emphasis on Cannabidiol and Ovarian Cancer
}

\author{
Lubabah. Ahmed, ${ }^{1}$ James. Evans, ${ }^{2}$ Pauric. Bannigan, ${ }^{3}$ Lie Yun. Kok, ${ }^{1}$ Christine. Allen, ${ }^{4}$ \\ ${ }^{1}$ Graduate Student, University of Toronto, Toronto, Canada \\ ${ }^{2}$ Project Manager, University of Toronto, Canada \\ ${ }^{3}$ Post Doctoral Fellow, University of Toronto, Canada \\ ${ }^{4}$ Professor, University of Toronto, Toronto, Canada
}

Purpose: There has been an increased interest in determining the therapeutic potential of the nonpsychoactive cannabinoids, cannabichromene (CBC), cannabidiol (CBD), and cannabigerol (CBG). To date, limited data is available on the anticancer effects that these compounds exert and how they may affect the efficacy of existing therapeutics when administered concurrently. The purpose of this study was to investigate the cytotoxicity of non-psychoactive cannabinoids in various cancer cell lines and to determine the combined drug effect of CBD with ovarian chemotherapeutic agents, cisplatin (CDDP), and paclitaxel (PTX) in ovarian cancer (OC) cell lines. Methods: CBC, CBD, and CBG were screened for cytotoxicity as monotherapy treatments against a panel containing seven cancer cell lines. CBD was further evaluated in combination with ovarian therapeutic agents in OC cell lines at various molar ratios of CBD to either CDDP or PTX. Cytotoxicity was evaluated using the acid phosphatase assay following 72 -hour treatment with either the individual cannabinoid or drug combination. To determine the combined drug effects, combination index values were calculated using the Compusyn software. Results: All three cannabinoids exhibited dose-dependent cytotoxic effects, which resulted in micromolar IC50 values. The most potent cannabinoid was CBD, with IC50 values ranging between $14.2 \pm 1.3$ and $24.3 \pm 3.8 \mu \mathrm{M}$. When CBD was further evaluated in combination with CDDP, the combined drug effect was neither cell line nor molar ratio dependent and was predominantly antagonistic at most molar ratios of CBD:CDDP. Conversely, the effects of CBD combined with PTX resulted in synergistic, additive, and antagonistic effects depending on the cell line evaluated. Conclusion: These results: illustrate that cannabinoids have cytotoxic potential against various cancer cell lines. Furthermore, CBD may potentiate or attenuate the effects of ovarian chemotherapeutic agents in vitro. Therefore, further investigation of combined drug effects in vivo is warranted. 


\title{
Effect of doxorubicin on catabolism of adenosine 5' -triphosphate and guanosine 5' -triphosphate in red blood cell in rats
}

\author{
Pollen Yeung, ${ }^{1}$ Sheyda Mohammadizadeh, ${ }^{2}$ Kelsey Mann, ${ }^{3}$ Remigius U. Agu, ${ }^{4}$ \\ ${ }^{1}$ Professor, Dalhousie University, Halifax, Canada; ${ }^{2}$ Graduate Student, Dalhousie University, Canada \\ ${ }^{3}$ Pharmacist, Dalhousie University, Canada; ${ }^{4}$ Professor, Dalhousie University, Canada
}

Purpose: Previous studies have shown catabolism of adenosine 5'-triphosphate (ATP) in red blood cells (RBC) is a potential surrogate biomarker for serious cardiovascular toxicity. In this study we compared the effect of doxorubicin (DOX) on breakdown of ATP versus GTP in RBC using a freely moving rat model. Method: Sprague Dawley (SD) rats $(\mathrm{n}=8-11)$ were each given either $10 \mathrm{mg} / \mathrm{kg}$ of DOX or normal saline by subcutaneous injection 2 times a day for 4 doses. Blood samples were collected just before the last injection (T0) and also at 0.08, 0.25, 1, 1.25, 1.5, 2, 3, 4, 5, and 6 hours after the last dose for measuring RBC concentrations of ATP, GTP and their catabolites. Area under the curve (AUC) after the last dose was calculated by trapezoidal method. Difference of ATP and GTP catabolism was assessed by unpaired t-test and considered significant at $\mathrm{p}<0.05$. Results: RBC concentrations of GTP and GDP measured at T0 were both significantly higher in the DOX treated rats compared to control (GTP $0.132 \pm 0.016$ vs $0.091 \pm 0.012$ $\mathrm{mM}$; GDP $0.083 \pm 0.0061$ vs $0.048 \pm 0.0071 \mathrm{mM}$, p < 0.05 for both). Catabolism of GTP as measured by AUC ratio of GDP / GTP was significantly greater in the DOX treated rats $(0.516 \pm 0.045$ vs $0.341 \pm 0.036$, $p=0.0072)$. The effect of DOX on ATP catabolism was not statistically significant $(p>0.05)$. Conclusion: DOX $(10 \mathrm{mg} / \mathrm{kg})$ given twice daily for 4 doses induced significant breakdown of GTP to GDP but not ATP to ADP in the RBC suggesting that GTP catabolism in the RBC is a more sensitive biomarker for cardiotoxicity induced by DOX (supported in part by funding from Dalhousie Faculty of Health and Pharmacy Endowment Foundation).

\section{Development of Physiologically Based Pharmacokinetic Model of Risperidone and Extrapolation to Different Ethnic Groups and Special Population}

\author{
Walaa Mahdy, ${ }^{1}$ Kazuhiro Yamamoto, ${ }^{2}$ Tomohiro Omura, ${ }^{3}$ Ikuko Yano, ${ }^{4}$ \\ ${ }^{1}$ Graduate Student, Department of Pharmacy, Kobe University Hospital, Canada \\ ${ }^{2}$ Lecturer, Department of Pharmacy, Kobe University Hospital, Kobe, Japan \\ ${ }^{3}$ Associate Professor, Department of Pharmacy, Kobe University Hospital, Japan \\ ${ }^{4}$ Professor, Department of Pharmacy, Kobe University Hospital, Japan
}

Purpose: Physiologically based pharmacokinetic (PBPK) analyses have been frequently used in the clinical pharmacology section of regulatory applications. However, whether models developed and validated in healthy populations can be extrapolated to untested populations is not well known. This study aimed to determine whether a drug-specific PBPK model validated in a healthy population could be used to predict drug disposition in populations with different ethnicities, ages, genetic phenotypes, and pregnant population considering an example for risperidone and its active metabolite, paliperidone. Methods: PBPK modeling and simulation were performed using Simcyp Population-based ADME Simulator version 20. The risperidone and paliperidone compound models were developed based on physicochemical and pharmacokinetic parameters reported in the literature. The model was validated using observed values from 25 clinical studies including 15 in the adult population (8 Caucasian, 5 Chinese, and 2 Japanese), 8 in the pediatric population (5 Caucasian, 2 Chinese, and 1 Japanese), and 2 in the pregnant population. Visual predictive checks (VPC) for predicted and observed plasma concentrations, goodness-of-fit plots, prediction fold error, mean error (ME), and root-mean-square error (RMSE) were used for the graphical and statistical analyses. Results: Almost all (98.9\%) of observed serum risperidone and paliperidone concentrations were 
within the $90 \%$ prediction intervals of each concentration by the PBPK simulation. All predicted values of serum risperidone concentration and AUC values in adult, pediatric, and pregnant populations met the 2fold acceptance criterion. A $91.8 \%$ of predicted values of paliperidone concentration and $97.4 \%$ of AUC values fall within the 2 -fold criterion in all populations. The mean error (ME) and root-mean-square error (RMSE) for all predicted Cmax for risperidone were $0.61 \pm 0.4 \%$, and $2.85 \%$, respectively. Regarding paliperidone, the values were $-0.038 \pm 0.45 \%$ and $3.16 \%$, respectively. Conclusion: This study successfully shows an experimental application of PBPK modeling for the adaptation of ethnic variety and pediatric population.

\title{
Prediction of HIV drug resistance by using 3D molecular field mapping
}

Fumiyoshi. Yamashita, ${ }^{1}$ Ryosaku. Ohta,${ }^{2}$ Kanako. So, ${ }^{3}$ Masahiro. Tsuda,,${ }^{4}$ Yuriko. Higuchi, ${ }^{5}$

${ }^{1}$ Professor, Kyoto University, Graduate School of Pharmaceutical Sciences, Kyoto, Japan

${ }^{2}$ Postdoc, Kyoto University, Graduate School of Pharmaceutical Sciences, Canada

${ }^{3}$ Junior Assistant Professor, Kyoto University, Graduate School of Pharmaceutical Sciences, Canada

${ }^{4}$ Assistant Professor, Kyoto University, Graduate School of Pharmaceutical Sciences, Canada

${ }^{5}$ Associate Professor, Kyoto University, Graduate School of Pharmaceutical Sciences, Canada

Purpose: Drug-resistant viruses have a significant impact on the prognosis of HIV infection. If drug resistance can be predicted based on the genotype, it is expected that appropriate drugs can be selected and efficient treatment can be provided. In the past, classification and regression models using various machine learning methods (support vector machine, deep learning, etc.) have been reported. However, these models have problems associated with data management. We develop a method of appropriate data weighting based on conditional probability theory and also propose a novel 3D structure-based modeling approach. Methods: We obtained HIV-1 drug resistance data from Stanford University's HIV Drug Resistance Database. The data set include incomplete sequencing data of HIV protease variants. With reference to the concept of population genetics, we computed the probability of occurrence of each possible mutant according to Bayesian conditional mutual information theory. On the other hand, we encoded the structural information of HIV-1 mutants as 3D molecular field parameters by homology modeling, structural alignment, and molecular field mapping. Results: When partial least squares, random forest, LightGBM, and support vector regression were employed to construct the prediction models, they all provided high prediction performance against drug resistance, where LightGBM was slightly better than the others. Partial least squares could also provide a contour map of molecular fields which indicate structural impacts on drug resistance acquisition. Conclusion: These physicochemically relevant models would be of use to design new inhibitors against drug-resistant HIV variants.

\section{Involvement of P-gp and BCRP in the transfer of integrase inhibitors to the brain}

\author{
Masahiro Tsuda, ${ }^{1}$ Mizuki Uno, ${ }^{2}$ Kanako So, ${ }^{3}$ Yuriko Higuchi, ${ }^{4}$ Fumiyoshi Yamashita, ${ }^{5}$ \\ ${ }^{1}$ Assistant Professor, Kyoto University, Graduate School of Pharmaceutical Sciences, Canada \\ ${ }^{2}$ Graduate Student, Kyoto University, Graduate School of Pharmaceutical Sciences, Canada \\ ${ }^{3}$ Junior Assistant Professor, Kyoto University, Graduate School of Pharmaceutical Sciences, Canada \\ ${ }^{4}$ Associate Professor, Kyoto University, Graduate School of Pharmaceutical Sciences, Canada \\ ${ }^{5}$ Professor, Kyoto University, Graduate School of Pharmaceutical Sciences, Kyoto, Japan
}

Purpose: Integrase inhibitors, key drugs for HIV treatment, have been associated with several neuropsychiatric adverse events, such as headache, insomnia, and depression, which have attracted attention in relation to the transfer of integrase inhibitors to the brain. Drug transfer to the central nervous system is 
restricted by the blood-brain barrier, where efflux transporters such as P-glycoprotein (P-gp) and breast cancer resistant protein (BCRP) play an important role. Integrase inhibitors are known to be substrates for these transporters in vitro, but their contribution in vivo is unclear. Therefore, we evaluated the involvement of P-gp and BCRP in the transfer of representative integrase inhibitors, dolutegravir and raltegravir, to the brain. Methods: Dolutegravir and raltegravir were administered intravenously to male ICR mice, and brain and blood concentrations were measured using LC-MS/MS. The effect of preadministration of oral elacridar, a dual inhibitor of P-gp and BCRP, on the brain and plasma concentrations of these two drugs were examined. Results: The brain-to-plasma concentration ratio was calculated to be 1.5 -fold higher for dolutegravir and 1.8-fold higher for raltegravir in the elacridal pre-treatment group than in the vehicle group, indicating an increase in the transfer of these drugs to the brain. The blood concentration of dolutegravir at 8 hours post intravenous administration was significantly increased by preadministration of elacridal. By contrast, the effect of elacridal preadministration on the blood concentration of raltegravir was negligible. Conclusion: These results suggested that transport via P-gp and BCRP is involved in the transfer of dolutegravir and raltegravir to the brain.

\section{Platelets Stimulate Programmed Death-Ligand 1 Expression by Cancer Cells: Inhibition by Anti- Platelet Drugs}

Amir Asgari, ${ }^{1}$ Gabriela Lesyk, ${ }^{2}$ Erika Poitras, ${ }^{3}$ Natasha Govindasamy, ${ }^{4}$ Kara Terry, ${ }^{5}$ Rachel To, ${ }^{6}$ Valentina Back, ${ }^{2}$ Jan Rudzinski, ${ }^{4}$ John Lewis, ${ }^{7}$ Paul Jurasz,${ }^{8}$

${ }^{1}$ Ph.D. student, Faculty of Pharmacy and Pharmaceutical Sciences, University of Alberta, Edmonton, Canada; ${ }^{2}$ graduated Student, Faculty of Pharmacy and Pharmaceutical Sciences, University of Alberta, Canada; ${ }^{3}$ Graduated student, Department of Pharmacology, University of Alberta, Canada; ${ }^{4}$ Graduated student, Department of Oncology, University of Alberta, Canada

${ }^{5}$ undergrad Student, Department of Pharmacology, University of Alberta, Canada; ${ }^{6}$ undergrad Student, Faculty of Pharmacy and Pharmaceutical Sciences, University of Alberta, Canada

${ }^{7}$ Associate Professor, Department of Oncology, University of Alberta, Canada; ${ }^{8}$ Associate Professor, Faculty of Pharmacy and Pharmaceutical Sciences, University of Alberta, Canada

Purpose: Platelets help facilitate hematogenous metastasis in part by promoting cancer cell immunoevasion, although our understanding of platelet function in modulating the adaptive immune system in cancer is limited. A major negative regulator of the adaptive response is the immune checkpoint protein Programmed Death Ligand 1 (PD-L1). Aim: As platelets secrete factors that may increase PD-L1 expression, we investigated whether they up-regulate cancer cell PD-L1, thus promoting immunoevasion, and whether common anti-platelet drugs inhibit this process. Methods: Platelets were isolated from human volunteers. A549 lung, PD-L1 null A549, and 786-O renal cancer cells were incubated with and without platelets, and cancer cell PD-L1 expression was measured by qPCR and flow cytometry. Additionally, platelet-cancer cell incubations were performed in the presence of common anti-platelet drugs, and with growth factor neutralizing antibodies. Following incubation with platelets, A549 cells were co-cultured with Jurkat cells and interleukin-2 (IL-2) levels were measured by flow cytometry as a marker of T-cell activation. Results: Platelets increased PD-L1 mRNA and surface protein expression by A549 and 786-0 cells. Combined neutralization of VEGF and PDGF prevented the platelet-induced up-regulation of PD-L1 by A549 cells, as did the anti-platelet drug eptifibatide. A549 incubated with platelets demonstrated a reduced ability to activate Jurkat cells and this effect was reversed by eptifibatide. Conclusion: As platelets promote immunoevasion of the adaptive immune response by increasing cancer cell PD-L1 expression and as anti-platelet drugs prevent this immunoevasive response, the investigation of anti-platelet drugs as adjuvant therapy to immune checkpoint inhibitors may be warranted in the treatment of cancer. 


\title{
Pharmacological Characterization of eNOS-based Megakaryocyte Subpopulations and Its Implications for their Platelet Progeny
}

\author{
$\underline{\text { Amir. Asgari, }}{ }^{1}$ Paul. Jurasz, ${ }^{2}$
}

${ }^{1}$ Ph.D. student, Faculty of Pharmacy and Pharmaceutical Sciences, University of Alberta, Edmonton, Canada

${ }^{2}$ Associate Professor, Faculty of Pharmacy and Pharmaceutical Sciences, University of Alberta, Canada

Purpose: Platelets play a key role in atherothrombosis and its associated inflammation. Recently, we identified platelet and megakaryocyte/blast subpopulations based on endothelial nitric oxide synthase (eNOS) and nitric oxide (NO) production. We showed that eNOS-negative (eNOSneg) platelets initiate aggregate formation, while eNOS-positive (eNOSpos) platelets limit thrombus growth. However, it's unknown how these platelet and megakaryocyte subpopulations arise and the impact of antiatherothrombotic drugs on their formation. Therefore, we aimed to (1) investigate whether proinflammatory cytokines known to down-regulate eNOS expression decrease the ratio of eNOSpos to eNOSneg megakaryocytes/blasts and resulting platelets; and (2) whether anti-atherothrombotic drugs, with known anti-inflammatory properties would counteract the effects of pro-inflammatory cytokines on eNOS expression and eNOS-based megakaryocyte and platelet subpopulation formation. Methods: Meg-01 were cultured in the presence of thrombopoietin and pro-inflammatory cytokines (IL-1 $\beta$, IL-6, TNF- $\alpha$, IFN- $\gamma$ ) and anti-atherothrombotic drugs acetylsalicylic acid (ASA $10-30 \mathrm{mM})$ and atorvastatin $(0.03-$ $1 \mathrm{mM})$. The levels of eNOSneg and eNOSpos Meg-01 and platelet-like particles was measured using flow cytometry. Results: Compared to control the cytokine cocktail significantly increased the percentage of eNOSneg Meg-01 and this effect was inhibited by $30 \mathrm{mM}$ ASA and $0.1 \mathrm{mM}$ atorvastatin (Control $7.4 \pm$ $0.9 \%$ vs cytokines $11.6 \pm 1.2 \%$ vs cytokines \& ASA and Control $9.4 \pm 1.1 \%$ vs cytokines $8.0 \pm 0.6 \%$ vs cytokines \& atorvastatin). Similarly, although fewer in their relative number compared to their parent Meg01, platelet-like particles released from eNOSpos Meg-01 cells decreased in response to inflammatory cytokines and this effect was reversed by ASA and atorvastatin. Conclusions: The generation of eNOSneg and eNOSpos megakaryocytes and platelets may be counter-regulated by inflammatory status. Conversely, anti-atherothrombotic drugs ASA and atorvastatin may promote an anti-thrombotic phenotype, in part, by increasing the formation of eNOSpos megakaryocytes and platelets.

\section{Inflammation-induced peripheral hyperalgesia perturbs neuronal function in the superficial dorsal horn of the spinal cord.}

Jenny Cheung, ${ }^{1}$ Samuel Fung, ${ }^{2}$ Hantao Zhang, ${ }^{2}$ Robert Bonin, ${ }^{3}$

${ }^{1}$ Undergraduate Student, Leslie Dan Faculty of Pharmacy, University of Toronto, Canada

${ }^{2}$ Graduate Student, Leslie Dan Faculty of Pharmacy, University of Toronto, Canada

${ }^{3}$ Assistant Professor, Leslie Dan Faculty of Pharmacy, University of Toronto, Canada

Purpose: Chronic pain affects more than 1.9 billion people worldwide and a significant proportion of these individuals suffer from chronic inflammatory pain, such as arthritis and myositis. The superficial dorsal horn (SDH) of the spinal cord is a key processing center for nociceptive signals sent from peripherally affected tissue. While much progress has been made to understand this phenomenon in locally affected tissue, putative alterations to the SDH are still poorly understood. Methods: We injected Complete Freud's Adjuvant (CFA), a known model for chronic inflammatory pain, into the right hind paw to study central changes in the SDH. We performed immunofluorescence staining with c-Fos and Ibal to investigate activation of ipsilateral SDH neurons and microglia compared to contralateral SDH neurons by confocal fluorescence microscopy. Calcium imaging of ipsilateral SDH and contralateral SDH neurons was 
performed following bath application of $10 \mu \mathrm{M}$ glutamate. Results: We observed a significant increase in neuronal activation on the ipsilateral side of the SDH compared to the contralateral side. We did not find any changes in microglial activation suggesting a lack of microglial involvement known to be present in neuropathic pain models. Calcium imaging demonstrated a greater evoked magnitude of activation of ipsilateral SDH neurons following glutamate application. Conclusion: Together, these findings suggest that peripheral induction of chronic inflammatory pain can alter neuronal activity in the SDH, which may contribute to the protracted nature of this model.

\section{Identifying Novel Allosteric Sites and Analyzing the Hydrogen Bond Networks and Correlated Dynamics in the SARS-CoV-2 RNA Polymerase}

$\underline{\text { Minwoo Ha }},{ }^{1}$ Khaled Barakat, ${ }^{2}$ Marawan Ahmed, ${ }^{3}$ Yasser Tabana, ${ }^{4}$

${ }^{1}$ Research Assistant, ${ }^{2}$ Assistant Professor, ${ }^{3}$ Postdoctoral Fellow, ${ }^{4} \mathrm{PhD}$ Student, Faculty of Pharmacy and Pharmaceutical Sciences, University of Alberta, Canada

Purpose: SARS-CoV-2 is the causative agent of COVID-19. Inhibition of the SARS-CoV-2 replicase machinery has been proven recently1 as a promising approach in combating the virus. Despite this, there are still several aspects related to the structure, function, and dynamics of the CoV-2 polymerase that need to be addressed. This study aims to further characterize the polymerase complex using in-silico analysis. Methods: Recently resolved SARS-CoV-2 polymerase cryo-EM structures2 were used as a starting point to construct the RdRp assembly. Active site-bound systems of the polymerase complex were constructed representing the incorporation of RNA nucleotides in the nascent chain. After adjusting for physiological conditions, classical MD simulations ( $100 \mathrm{~ns})$ and accelerated MD simulations were conducted. Statistical analysis of the collected coordinates was done to characterize the conformational dynamics, hydrogen bonding, water hydration shell, free energy, and druggability of the systems. Results: This is the first study to build the SARS-CoV-2 replicase complex, providing deep insight into its dynamicity. Our findings provide a detailed analysis of the hydrogen bond networks at various parts of the polymerase structure and suggest possible nucleotide substitutions that can be tolerated by the polymerase complex. Three druggable allosteric sites within the nsp12 RdRp that can be targeted by small molecule inhibitors are reported. Our correlated motion analysis shows that the dynamics within one of the newly identified sites are linked to the active site, indicating that targeting this site can significantly impact the catalytic activity of the SARS-CoV-2 polymerase. Conclusions: MD simulations and subsequent state-ofthe-art data analysis were used to study the properties of the various polymerase subdomains under physiological conditions. This study provides a rational basis for the discovery of new drug interventions against SARS-CoV-2.

\section{Investigating the influence of $\mathrm{N}$-glycosylation on the intracellular location of human UDP- glucuronosyltransferase 1A6 (UGT1A6)}

\section{Yuejian Liu,${ }^{1}$ Michael Coughtrie, ${ }^{2}$ Abby Collier, ${ }^{3}$}

${ }^{1} \mathrm{PhD}$ candidate, Collier and Coughtrie Laboratories, Faculty of Pharmaceutical Sciences, The University of British Columbia, Vancouver, Canada; ${ }^{2}$ Professor and Dean, Faculty of Pharmaceutical Sciences, The University of British Columbia, Canada; ${ }^{3}$ Professor; Director of Bachelor of Pharmaceutical Sciences degree program, Faculty of Pharmaceutical Sciences, The University of British Columbia, Canada

Purpose: UDP-glucuronosyltransferases (UGTs) mediate the biotransformation of numerous endo- and xenobiotics and are integral to vertebrate detoxication. Insufficient glucuronidation can lead to congenital syndromes, chemical toxicities, and dysregulated hormonal status. The UGTs undergo post-translational 
modifications at the endoplasmic reticulum (ER) membrane, which may affect the intracellular transport and the function of the enzymes. This study aimed to investigate the influence of $\mathrm{N}$-glycosylation on the cellular localization of human UGT1A6, one of the most important UGT isoforms. Methods: 4 recombinant HEK293 cell lines were established to express UGT1A6 N-glycosylation variants where 2 possible N-glycosylation sites were removed, namely the wild-type, single mutants N294Q and N346Q, and the double mutant. Immunocytochemistry followed by confocal imaging was performed to detect each UGT1A6 variant, calnexin (an ER marker), and the nucleus $(\mathrm{n}=3)$. Western blotting was used to compare UGT1A6 expression between microsomes and cytosol, including calnexin dual detection. UGT1A6 and calnexin were also compared between microsomes and the $\mathrm{S} 9$ waste pellet to examine microsome recovery $(n=3)$. Results: Confocal images demonstrated all N-glycosylation variants colocalized with calnexin at the ER, a finding supported by western blot data where UGT1A6 and calnexin were observed in the microsomes but not the cytosol. However, UGT1A6 and calnexin were also detected in S9, indicating that microsome recovery from cellular organelles was incomplete. Conclusion: N-glycosylation did not alter the intracellular localization of UGT1A6 to the ER membrane. In the future, UGT1A6 can be examined by electron microscopy to compare the $\mathrm{N}$-glycosylation variant localization between rough and the smooth ER where functional enzyme locates. Investigating the intracellular localization of UGTs provides insights on how the post-translational modifications regulate their structure and function, which is critical for understanding the fundamental mechanisms of glucuronidation.

\section{Infection and twin pregnancy are associated with altered maternal-placental-fetal steroid levels in both normal conception and assisted reproduction.}

\section{Hayley Price, ${ }^{1}$ Cecilia Jalabert, ${ }^{2}$ Chunqui Ma, ${ }^{3}$ Kiran Soma, ${ }^{4}$ Michael Coughtrie, ${ }^{5}$ Abby Collier, ${ }^{6}$}

${ }^{1} \mathrm{PhD}$ Candidate, ${ }^{2} \mathrm{PhD}$ Student, The University of British Columbia, Canada; ${ }^{3}$ Lab Manager, The University of British Columbia, Canada; ${ }^{4}$ Professor, The University of British Columbia, Canada; ${ }^{5}$ Professor and Dean, Faculty of Pharmaceutical Sciences, The University of British Columbia, Canada; ${ }^{2}$ Professor; Director of Bachelor of Pharmaceutical Sciences degree program, Faculty of Pharmaceutical Sciences, The University of British Columbia, Canada

Purpose: Assisted reproduction technologies (ART) are used to treat infertility and have become increasingly common in recent decades. Although considered safe and successful, pregnancies conceived using ART require more obstetric interventions and show higher risk of complications. While the underlying reason for these complications is unknown, placental abnormalities have been reported in ART and may be part of the mechanism. Previously, we showed altered placental steroid metabolism and delivery in a mouse ART model, and here aimed to determine if these differences are reflected in human pregnancy. Methods: The steroids pregnenolone, progesterone, androstenedione, dehydroepiandrosterone, estrone, estradiol, estriol, and cortisol were measured using UHPLC-MS/MS in placentas from 112 naturally conceived and ART pregnancies, with matching maternal and cord serum where available. The method is accurate, precise, and highly sensitive, requiring $1.5 \mathrm{mg}$ of tissue $/ 10 \mu \mathrm{L}$ of serum, with a linear range from 0.2-10,000 pg per sample. Steroid levels were compared between natural conception and ART, and subsequently stratified using clinical outcome data, including influence of age, BMI, and sex. Results: All steroids were detected with the exception of placental dehydroepiandrosterone and maternal pregnenolone. No differences were observed between natural conception and ART in maternal, placental, or fetal samples. Once stratified by obstetric outcome, two novel observations were observed. In twin pregnancies, androstenedione, estrone, and cortisol levels were significantly lower compared to singletons ( $\mathrm{p}<0.05$ ). In pregnancies complicated with chorioamnionitis, androstenedione, estrone, estradiol, and pregnenolone levels were significantly higher compared to uncomplicated pregnancies $(\mathrm{p}<$

0.05). Conclusion: These data suggest changes in steroidogenesis and metabolism in response to multiple birth and infection. Future work evaluating steroidogenic/clearance enzymes will provide mechanistic 
insight into these associations. Although steroid manipulation in pregnancy has a chequered past in pharmacology and medicine, careful evaluation of these pathways may present a therapeutic target for preventing complications associated with multiple births and infection.

\title{
Studying the Aryl Hydrocarbon Receptor (AhR) ligand-binding domain in a computational approach
}

\author{
Ayman O.S. El-Kadi, ${ }^{1}$ Khaled. Barakat, ${ }^{2}$ Farag. Mosa, ${ }^{3}$ \\ ${ }^{1}$ Professor, Faculty of Pharmacy and Pharmaceutical Sciences, University of Alberta, Edmonton, Canada \\ ${ }^{2}$ Assistant Professor, Faculty of Pharmacy and Pharmaceutical Sciences, University of Alberta, Canada \\ ${ }^{3} \mathrm{PhD}$ student, Faculty of Pharmacy and Pharmaceutical Sciences, University of Alberta, Canada
}

Purpose: The aryl hydrocarbon receptor (AhR) is a ligand-activated transcriptional factor. It regulates various genes' expression and plays a pathophysiological function in numerous diseases, including cancer progression and cardiovascular diseases. The full-length AhR encompasses various domains, including bHLH, PAS A, PAS B \& transactivation domains. The PAS B domain plays a crucial role in regulating the activity of AhR by interacting with small molecules through its ligand-binding domain (LBD). Here we used computational modelling to study LBD structure and understand how AhR ligands interact with residues in LBD. Methods: The crystal structure of the PAS B domain from mouse and human is not available; hence, we used different homology modelling algorithms to build their 3-dimensional (3-D) structures. The models were then refined using molecular dynamics (MD) simulations, followed by data mining and clustering analysis to extract their most dominant conformations. Compounds were then tested for their binding to the LBD using molecular docking simulations. Results: Structures for human hypoxiainducible factors (HIF-2 $\alpha$ ) and (HIF-1 $\alpha$ ) were used as templates to build different models for the PAS B domain. HIF-1/2 $\alpha$ showed $\sim 31 \%$ sequence similarities to PAS B. 17 different PAS B models were built using different homology modelling algorithms and were evaluated using the Ramachandran plot. The most dominant conformations from human and mouse models represent $83 \%$ and $65 \%$, respectively. Two models showed acceptable scores and were used to test Several AhR modulators' binding containing Dioxin and CH-223191 in the AhR- LBD. Conclusion: Molecular docking predicts how AhR-LBD interacts with AhR modulators in their binding pocket and identifies the important residues that interact with the different AhR ligands. Our study can advance the development of new AhR antagonists for the treatment of cancer and cardiovascular diseases.

\section{Fishing the target of a potent small molecule immunomodulator for cancer immunotherapy}

Yasser Tabana ${ }^{1}$, Dinesh Babu ${ }^{1}$, Marawan Ahmed ${ }^{1}$, Garett Dunsmore ${ }^{3}$, Shima Shahbaz ${ }^{2}$, R Piragasam ${ }^{4}$, Richard Fahlman ${ }^{4}$, Tae Chul moon ${ }^{1}$, Shokrollah Elahi ${ }^{2,3}$, Arno Siraki ${ }^{1}$, Khaled Barakat ${ }^{1}$,

Faculty of Pharmacy \& Pharmaceutical Sciences, University of Alberta, Edmonton, Canada

Purpose: Cancer immunotherapy has emerged as the fourth pillar of cancer treatment along with surgery, radiation, and chemotherapy. Immunotherapeutic approaches utilize components of a patient's own immune system to selectively target cancer cells. Our lab has identified a small molecule (Compound-A) that boosts $\mathrm{T}$-cell proliferation and cytokine production. A comprehensive investigation is ongoing to identify and validate the target(s) and pathway(s) of this compound that contribute to its activity on T-cell proliferation and cytokine production. Methods: The effect of compound-A on its ability to produce cytokines (IFN $\gamma$ and IL-2) and increase the T-cell proliferation in peripheral blood mononuclear cells (PBMCs) were measured by ELISA and CFSE staining, respectively. The genomic and proteomic changes were analyzed using RNA sequencing and label-free quantitative proteomics. The microsomal stability and toxicity of Compound-A 
against PBMCs were determined. Identification of the possible target(s) using pull-down was also conducted. Results: Compound-A increased T-cell proliferation and IL-2 secretion. Compound-A showed that $59.6 \%$ was remaining after 60 min of incubation with human hepatic microsomes. The CC50 observed against PBMCs was $20.6 \mu \mathrm{M}$. After treating PBMCs with Compound-A for $12 \mathrm{~h}$, a total of 792 differentially expressed genes (DEGs) were identified including 377 upregulated and 415 downregulated genes. Also, a total of 863 DEGs were identified after $24 \mathrm{~h}$ treatment, including 444 upregulated and 419 downregulated genes. GO and genome pathway analysis showed that these DEGs were enriched in signaling pathways associated with response to IFN $\gamma$. Plausible targets were obtained by pull-down assay, although they need further confirmation. Conclusion: Our study showed the immunostimulatory activities of Compound-A with possible immunological targets. A future direction will be to validate the molecular targets responsible for its immunological activities.

\section{Studying the effects of selective calcium channel blockers on ions' permeation through the Cav1.2 ion channel}

$\underline{\text { Farag Mosa, }},{ }^{1}$ Tianhua Feng, ${ }^{2}$ Suryanarayanan Chandrasekaran, ${ }^{3}$ Khaled Barakat, ${ }^{4}$

${ }^{1} \mathrm{PhD}$ student, Faculty of Pharmacy and Pharmaceutical Sciences, University of Alberta, Canada

${ }^{2}$ Master student, Faculty of Pharmacy and Pharmaceutical Sciences, University of Alberta, Canada ${ }^{3}$ postdoctoral, Faculty of Pharmacy and Pharmaceutical Sciences, University of Alberta, Canada

${ }^{4}$ Assistant Professor, Faculty of Pharmacy and Pharmaceutical Sciences, University of Alberta, Canada

Farag E.S. Mosa1, SuryanarayananC1, Tianhua Feng1, Khaled Barakat1 1Faculty of Pharmacy and Pharmaceutical Sciences, University of Alberta, Edmonton, AB, Canada Purpose: Selective calcium channel antagonists are commonly used in the treatment of cardiovascular disorders. They are mostly classified into 1,4-dihydropyridine (1,4-DHPs) and non-DHPs. The non-DHPs class is further classified into phenylalkylamines (PAAs) and benzothiazepines (BZTs) derivatives. These blockers are used for the treatment of hypertension, angina pectoris, and cardiac arrhythmias. Despite their well-established efficacy, the structural basis behind their activity is not very clear. Methods: In this study, we used a near-open confirmation (NOC) model of the Cav1.2 cardiac ion channel to examine the mode of binding of these antagonists within the pore domain and the fenestration of the pore-forming domains. Effects of calcium ion permeation in the presence of drug molecules were assessed using steered molecular dynamics (SMD) simulations. Results: These studies reveal that nicardipine, a DHP derivative, shows a strong Cav1.2 blocking activity, requiring more than 2500 piko Newton $(\mathrm{pN})$ force to pull calcium ions toward the channel's pore in the presence of the compound. Similar blocking activity was observed for verapamil, a PAA derivative, requiring almost $2300 \mathrm{pN}$ of force. The least blocking activity was observed for Diltiazem, a BZT derivative. Conclusion: Our results explain the structural basis and the binding details of 1,4-DHPs, PAAs, and BZTs at their distinct Cav1.2 sites and offer detailed insights into their mechanism of action in modulating the Cav1.2 channel.

\section{Treatment with glucagon-like peptide-1 agonists improves $\beta$-cell survival in amyloid forming islets during ex vivo culture.}

\section{$\underline{\text { Snehit Patel, }},{ }^{1}$ Lucy Marzban, ${ }^{2}$}

${ }^{1}$ Pharmacy Student, College of Pharmacy, University of Manitoba, Winnipeg, Canada

${ }^{2}$ Associate Professor, College of Pharmacy, University of Manitoba, Canada

Purpose: Diabetes is a chronic metabolic disease characterized by hyperglycemia. Type 2 diabetes (T2D) is caused by impaired insulin secretion and peripheral insulin resistance, while T1D is caused by auto-immune 
destruction of islet $\beta$-cells. Islet transplantation is a promising treatment approach for T1D but is currently limited by islet availability and poor long-term survival of islet grafts due to both immune and non-immune factors. Islet amyloid, formed by aggregation of $\beta$-cell hormone, human islet amyloid polypeptide (hIAPP), is a pathologic characteristic of T2D that contributes to $\beta$-cell loss. Amyloid also forms in pre-transplant cultured and transplanted islets and contributes to graft dysfunction. Glucagon-like peptide-1 (GLP-1) agonists, exenatide (short-acting) and liraglutide (long-acting), mimic GLP-1 action to enhance $\beta$-cell function in diabetes. We investigated if GLP-1 agonists can improve survival of islets during ex vivo culture by reducing amyloid-induced $\beta$-cell death, as a potential strategy to enhance islet quality and quantity during pre-transplant culture. Methods: Freshly isolated islets from hIAPP-expressing transgenic mice, an animal model of amyloid-associated T2D, were cultured to form amyloid similar to that in T2D and islet grafts. Free-floating cultured islets were treated with a vehicle, exenatide $(10 \mathrm{nmol} / \mathrm{L})$ or liraglutide $(10 \mathrm{nmol} / \mathrm{L}) . \beta$-cell apoptosis and amyloid formation in islets were assessed by quantitative immunolabelling for insulin and TUNEL or thioflavin S, respectively. Results: The rate of $\beta$-cell apoptosis increased in islets during culture which closely correlated with progressive amyloid formation. Treatment with either exenatide or liraglutide reduced amyloid formation and $\beta$-cell death in hIAPP-expressing islets with the greatest decrease being in liraglutide-treated islets. Conclusion: GLP-1 agonists may provide an effective approach to enhance islet survival during pre-transplant culture thereby improving long-term survival of islet grafts. Long-acting GLP-1 agonists may be more effective in protecting islets in conditions associated with amyloid formation such as T2D and pre-transplant cultured islets.

\title{
Uncovering Novel Routes of Folate Delivery in the Mouse Central Nervous System
}

\author{
Vishal Sangha, ${ }^{1}$ Reina Bendayan, ${ }^{2}$ Md Tozammel Hoque, ${ }^{3}$ Jeffrey Henderson, ${ }^{4}$ \\ ${ }^{1}$ Graduate Student, University of Toronto, Canada; ${ }^{2}$ Professor, University of Toronto, Canada \\ ${ }^{3}$ Senior Research Associate, University of Toronto, Canada; ${ }^{4}$ Associate Professor, University of Toronto, \\ Canada
}

Purpose: Folates are a family of B9 vitamins critical in neurodevelopment. In the central nervous system (CNS), folate transport is primarily mediated by folate receptor alpha (FR $\alpha)$ at the blood-cerebrospinal fluid barrier (BCSFB). Impairments in FR $\alpha$-mediated folate transport at the BCSFB can result in severe neurological deficits, therefore it is essential to characterize novel routes of brain folate transport. The objective of this study was to investigate the localization of the folate transport systems i.e., reduced folate carrier (RFC), proton-coupled folate transporter (PCFT), folate receptor alpha (FR $\alpha)$ in various brain cellular compartments. In particular, transporter/receptor localization was examined at the brain barriers [blood-brain barrier (BBB), BCSFB, arachnoid barrier (AB)] and in brain parenchyma (astrocytes, microglia, neurons). Methods: Applying immunohistochemical analysis, localization of RFC, PCFT, and FR $\alpha$ was examined in frozen brain sections of C57BL6/N wildtype mice utilizing appropriate specific antibodies and standard markers. Gene expression of the transporters/receptor was further characterized in primary neuronal cultures of CD1 mice, and in immortalized mouse AB cells using qPCR analysis. Results: Localization of all three folate transport systems was detected at the mouse BBB and $\mathrm{BCSFB}$, which is consistent with previous data. At the AB, astrocytes and neurons, localization of RFC and PCFT (but not FR $\alpha$ ) was observed. In contrast, transporter/receptor localization was not detected in microglia. Gene expression of RFC and PCFT was confirmed in neuronal cultures and in mouse AB cells Conclusion: Localization of the folate transporters (RFC, PCFT) at the AB may represent a novel route of brain folate delivery into the cerebrospinal fluid (CSF), with transporter expression in astrocytes and neurons further contributing to folate transport into brain parenchyma. In disorders associated with impaired FR $\alpha$-mediated transport such as cerebral folate deficiency, inducing RFC/PCFT functional expression at these various brain compartments may represent a novel treatment strategy. 


\title{
Glucagon-like peptide-1 agonists and interleukin-1 receptor antagonists have synergistic effects on reducing $\beta$-cell apoptosis and improving $\beta$-cell proliferation in amyloid-forming human islets
}

\author{
Mukta Moni, ${ }^{1}$ Maximilian Fidel, ${ }^{2}$ Lucy Marzban, ${ }^{3}$ \\ ${ }^{1}$ Graduate Trainee, ${ }^{2}$ Trainee, ${ }^{3}$ Associate Professor, College of Pharmacy, Rady Faculty of Health Sciences, \\ University of Manitoba, Winnipeg, Canada;
}

Purpose: A key factor contributing to $\beta$-cell death in Type-2 Diabetes (T2D) is amyloid formation in pancreatic islets. Amyloid also forms in cultured and transplanted islets in patients with Type-1 Diabetes (T1D), potentially leading to graft failure. Glucagon-like peptide-1 (GLP-1) agonists (exenatide) and interleukin-1 receptor antagonists (anakinra) improve $\beta$-cell survival, proliferation, and function, thereby improving hyperglycemia in diabetes. We examined the synergistic beneficiary effects of anakinra and exenatide on amyloid-induced $B$-cell death and reduced $B$-cell proliferation in human islets, as a potential therapeutic strategy to protect $\beta$-cells from amyloid toxicity in T2D and islet grafts in T1D. Methods: Human islets from cadaveric pancreatic donors $(n=4$; purity $>90 \%)$ were cultured free-floating in CMRL with elevated $(11.1 \mathrm{mmol} / \mathrm{l})$ glucose for 7 days (to form amyloid), with or without exenatide (10nmol/l), anakinra $(10 \mu \mathrm{g} / \mathrm{ml})$ or both. Culture medium was changed every 48 hours. Amyloid formation, $B$-cell apoptosis and proliferation were assessed by performing quantitative immunohistochemistry on paraffinembedded islets for insulin and thioflavin S, TUNEL, or PCNA, respectively. Results: Treatment with either exenatide or anakinra reduced the number of amyloid-positive islets during culture, with maximum effect observed by combined treatment (non-treated: $12 \pm 4 \%$, exenatide: $5 \pm 1 \%$, anakinra: $4 \pm 0.8 \%$, exenatide+anakinra: $2 \pm 0.5 \%$; $<0.05$ ). Reduced amyloid formation in exenatide or anakinra-treated islets closely correlated with lower rate of TUNEL-positive (apoptotic) $\beta$-cells and combined treatment was more effective than each treatment alone (non-treated: $7.4 \pm 0.9 \%$, exenatide: $3.6 \pm 0.5 \%$, anakinra: $3.9 \pm 0.7 \%$, exenatide+anakinra: $2.1 \pm 0.3 \%$; $<<0.05)$. Moreover, treatment with exenatide or anakinra increased the proportion of PCNA+(proliferative) $\beta$-cells by about 2 -fold in islets during culture which was further enhanced with combined treatment. Conclusion: These findings suggest that 1) $\beta$-cell protective effects of exenatide and anakinra are partially mediated by reducing amyloid formation and $\beta$-cell toxicity; 2 ) combined exenatide+anakinra treatment has synergistic effects on reducing $\beta$-cell apoptosis and enhancing $\beta$-cell proliferation in amyloid-forming human islets.

\section{Identifying Evidence-Based Pharmacy Practices for the Implementation of Pharmacogenomics Through A Scoping Review}

\author{
Meagan Hayashi, ${ }^{1}$ Dalia Hamdy, ${ }^{2}$ Sherif Mahmoud, ${ }^{3}$ \\ ${ }^{1} \mathrm{MSc}$. Candidate, ${ }^{2}$ Clinical Assistant Professor, ${ }^{3}$ Clinical Associate Professor, University of Alberta, \\ Canada
}

Purpose: Pharmacogenomics (PGx) can provide valuable pharmacokinetic and pharmacodynamic information for the pharmacist's assessment of drug therapy, especially within medication therapy management (MTM) services. However, no review has comprehensively mapped pharmacists' use of PGx in practice-based research. Doing so would allow future researchers, practitioners, and policy-makers to identify the ideal populations and settings for PGx implementation within the pharmacy. The purpose of this review is to identify the evidence to date of PGx use in pharmacy practice. Methods: A scoping review was conducted to find all studied non-oncologic pharmacy practices incorporating PGx testing. Search terms were applied to 5 databases and relevant journals. Characteristics of patients, pharmacy settings, genetic tests, and outcomes were summarized to determine models most likely to benefit patients. Results: The search identified 43 studies on the use of PGx by pharmacists published between 2007 and 2020 
(Figure 1). CYP2C19 testing with antiplatelets was the most studied model, found in both community and institutional settings. It also was the most actionable test: approximately $30 \%$ of patients have polymorphisms indicating a need for alternative antiplatelets, and identifying these patients can reduce morbidity and mortality by more than $50 \%$. As technology shifts, broader studies using multi-gene panel tests within MTM demonstrate an approximate 50\% decrease in emergency visits and hospitalizations in elderly polypharmacy patients. Clinical benefit or drug-gene interactions are also found in other cardiovascular, psychiatric, analgesic, and gastrointestinal indications. No evaluations of actual costs or of pharmacist prescribing were found in included literature. Facilitators towards successful PGx implementation included pharmacist education, collaboration with other healthcare providers, and the use of clinical decision software. Conclusion: PGx has demonstrated feasibility and improved medication outcomes in many indications within pharmacy practice. Further PGx research should be directed towards pharmacist prescribing, education, and pharmacoeconomics.

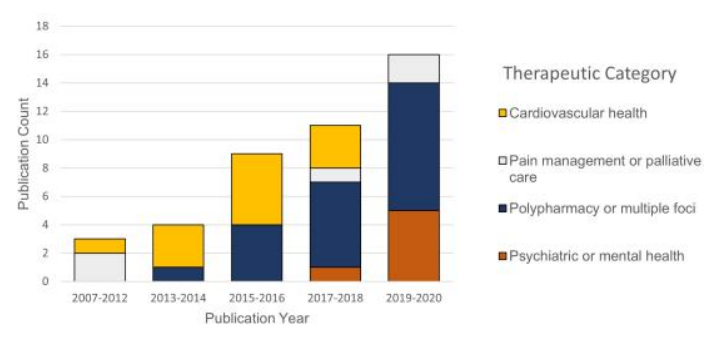

\title{
Characteristics that Influence Purchase Choice for Cannabis Products: A Systematic Review
}

\author{
Omar Shogan, ${ }^{1}$ Jennifer Donnan, ${ }^{2}$ Lisa Bishop,${ }^{3}$ Michelle Swab,${ }^{4}$ Maisam Najafizada, ${ }^{5}$ \\ ${ }^{1}$ Undergraduate Student Research Assistant, Memorial University of Newfoundland, Canada \\ ${ }^{2}$ Assistant Professor; Supervisor - Pharmacy, Memorial University of Newfoundland, Canada \\ ${ }^{3}$ Associate Professor; Memorial University of Newfoundland - Pharmacy, Canada \\ ${ }^{4}$ Public Services Librarian, Memorial University of Newfoundland, Canada \\ ${ }^{5}$ Assistant Professor; Memorial University of Newfoundland - Medicine, Canada
}

Purpose: When non-medical cannabis use became legal, Canadian governments implemented policies to encourage safe consumption through access to a regulated market. While this market is growing, sales still occur through unregulated channels. This systematic review identifies factors influencing cannabis purchasing to help policymakers understand why consumers still purchase black market cannabis. Methods: A comprehensive search strategy included databases in health, business and social science fields. Studies were eligible for inclusion if they were conducted with persons who purchase cannabis, and examine at least one attribute that would influence purchase choice. Studies could be of any methodological design. Two independent reviewers completed two levels of screening, and all extraction was verified by a second reviewer. A qualitative synthesis of the findings was completed. Results: Thirty publications were included. Aspects of price were the most common factors (24 studies). Eighteen studies measured price elasticity; most studies found that demand was price inelastic. Many other attributes were identified (e.g. product quality, route of administration, product recommendations, packaging), but none explored in depth. Eleven studies addressed aspects of product quality: demand elasticity, potency, aroma, etc. Studies also explored consumer-perceived "quality", but provided no definition; differences in quality appeared to impact consumer choice. Conclusion: While price influences choices, demand is relatively inelastic. This suggests that consumers may be seeking lowest-cost, unregulated cannabis without reducing 
consumption. Beyond price, there is a significant gap in our understanding of consumer choices. Though illdefined, quality's impact on choice indicates that we need a better understanding of it.

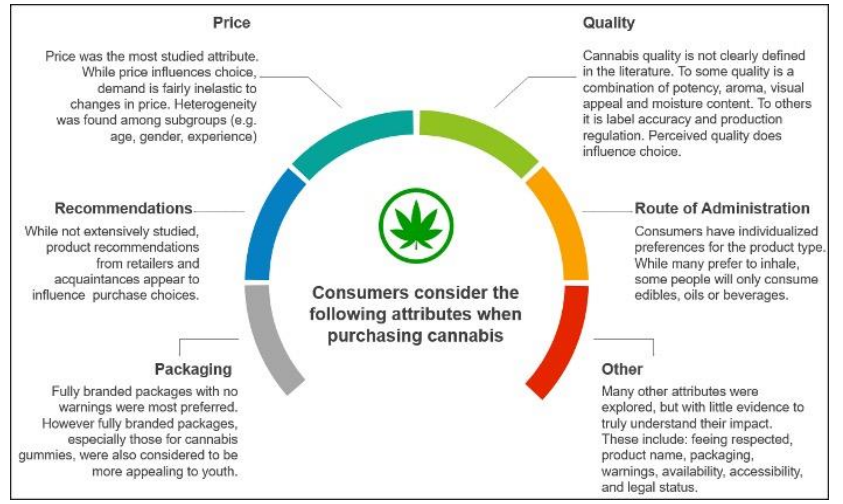

\section{Implementing an updated evidence-based asthma care pathway for pediatric inpatients: a pre-post quality improvement study}

James Davis, ${ }^{1}$ Melanie MacInnis, ${ }^{2}$ Joanna Holland, ${ }^{3}$ Jaqueline vanNieuwenhuizen, ${ }^{3}$ Jaime McDonald, ${ }^{2}$

${ }^{1}$ Student, Dalhousie University, Canada; ${ }^{2}$ Pharmacy Department, IWK Health Centre, Canada; ${ }^{3}$ Pediatric Medical Unit, IWK Health Centre, Canada

Purpose: To determine if a newly implemented asthma pathway reduced length of stay (LOS) at a pediatric tertiary care centre. Methods: This retrospective, pre-post quality improvement study was conducted on patients discharged from hospital with asthma. The pre- and post-implementation groups consisted of patients admitted from October 1, 2018 to July 31, 2019 and October 1, 2019 to July 31, 2020, respectively. Due to the COVID-19 pandemic, a post-implementation subgroup excluded admissions after March 1, 2020. The primary outcome was inpatient LOS. Results: There were 97 and 48 eligible encounters in the pre- and post-implementation groups, respectively. Baseline characteristics were similar. Average LOS was 41.3 (36.2 to 46.3) and 35.1 (29.9 to 40.3) hours ( $\mathrm{p}=0.246)$ for the pre- and post-implementation groups, respectively. There were $40(41.2 \%)$ and $17(35.4 \%)$ readmissions to hospital and/or ED ( $\mathrm{p}=0.500)$ and the average drug cost per visit was $\$ 10.67$ (8.13 to 13.22 ) and $\$ 13.13$ (5.51 to 17.73) ( $\mathrm{p}=0.566$ ). There was no difference in ICU admissions or transfer (12.7\% [16/126] vs. 9.8\% [6/61], p=0.569). The subgroup analysis revealed no significant differences for any outcomes. Conclusion: The updated protocol provides an evidence-based approach to treating asthma at our institution without any apparent safety or quality concerns.

\section{Lower Insoluble Aggregates of DJ1 and Parkin Proteins in the Substantia Nigra of Parkinson's Disease Patients}

\section{Genesis Lobo, ${ }^{1}$ Cyntia Tremblay, ${ }^{2}$ Frédéric Calon, ${ }^{3}$}

${ }^{1}$ Undergraduate student, Faculty of Pharmacy, Université Laval, Québec, QC, Canada, Canada; ${ }^{2} \mathrm{PhD}$ candidate, Neurosciences Unit, Centre de recherche du CHU de Québec - Université Laval, Québec, Qc, Canada, Canada; ${ }^{3}$ Academic supervisor, researcher, Faculty of Pharmacy, Université Laval, Québec, QC, Canada, Canada 
Purpose: Parkinson's disease is a debilitating movement disorder consequent of the loss of dopaminergic neurons. While its pathogenesis is not well known, proteinopathies in the substantia nigra pars compacta may be at cause. DJ1 and Parkin proteins are known protectors against proteinopathies. The general objective of this study is to quantify and compare DJ1 and Parkin post-mortem levels in the substantia nigra of Parkinson's disease patients $(n=24)$ and a control group $(n=21)$. Methods: We performed western blot analyses to quantify DJ1 and Parkin in fractions containing (i) detergent-soluble and (ii) detergent-insoluble proteins (formic acid extracts) from the substantia nigra of collected brain samples $(n=45)$. Protein concentration was determined using bicinchoninic acid assays. Proteins were electroblotted onto PVDF membranes before being immunoblotted with appropriate primary and secondary antibodies, then chemiluminescence reagents. The membranes were then placed onto the chemiluminescence and fluorescence imaging system. Optical density analyses were performed using Image Lab (Bio-Rad). For the statistical analyses, the non-parametric Mann-Whitney t-test was used to compare the control group with the Parkinson's disease substantia nigra samples. A p value $<0.05$ is considered statistically significant. Actin was used to normalize the detergent-soluble fractions. Results: Our preliminary results showed no significant difference in detergent-soluble DJ1 and Parkin levels in the substantia nigra between patients in the control group and those with Parkinson's disease. However, a decrease in detergent-insoluble DJ1 and Parkin was observed in Parkinson's Disease. Conclusion: The pathological significance of formic acidsoluble DJ1 and Parkin have yet to be studied, but alpha-synuclein aggregation into Lewy bodies is known to be a hallmark of Parkinson's disease. The cellular process underlying the conversion of DJ1 and Parkin from soluble to insoluble forms in Parkinson's disease warrants further studies.

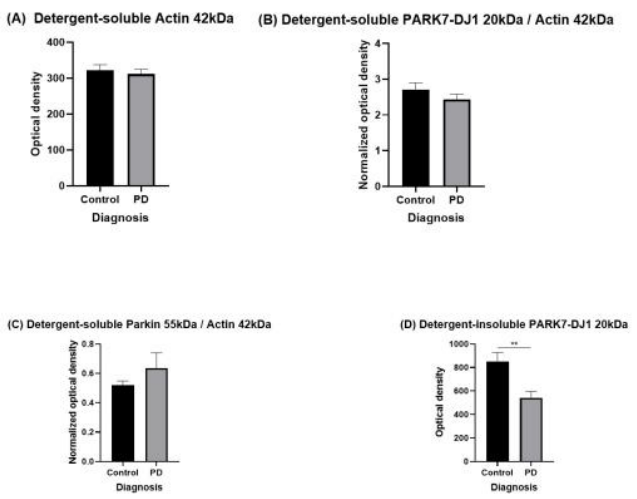

\title{
Functionalized Nanodiamonds as Diamoplexes for Gene Delivery: Rational Design, Physicochemical Characterization and Cellular Interaction Studies.
}

\author{
$\underline{\text { Saniya Alwani, }},{ }^{1}$ Raj Rai, ${ }^{2}$ Deborah Michel, ${ }^{3}$ Chithra Karunakaran, ${ }^{4}$ LaRhonda Sobochishin, ${ }^{5}$ Ildiko Badea,${ }^{6}$ \\ ${ }^{1}$ Student, College of Pharmacy and Nutrition University of Saskatchewan, Canada; ${ }^{2}$ Research Assistant, \\ College of Pharmacy and Nutrition University of Saskatchewan, Canada; ${ }^{3}$ Research Technician, College of \\ Pharmacy and Nutrition University of Saskatchewan, Canada; ${ }^{4}$ Beamline Scientist, Canadian Light Source, \\ Canada; ${ }^{5}$ Research Technician, College of Veterinary Medicine University of Saskatchewan, Canada; \\ ${ }^{6}$ Professor, College of Pharmacy and Nutrition University of Saskatchewan, Canada
}

Purpose: We utilized nanodiamonds (NDs) to design gene carriers for nucleic acid-therapeutics. NDs were functionalized with lysine to confer positive charge to the diamonds, for complexing anionic siRNA, forming diamoplexes. Histidine was added to improve the gene transfection efficiency of the system. Methods: Amino acids (AAs) were attached on NDs as in Figure1. 
Physicochemical assessments - infrared spectroscopy, thermogravimetry, particle size and zeta potential measurements and gel electrophoresis. Bioassays - transmission electron microscopy, scanning transmission x-ray microscopy (STXM) and flow cytometry. Results: Functionalization of NDs with lysine and lysylhistidine was evident with surface loadings of $1.97 \& 14.49$ moles/gm respectively. Our study is first to quantify the extent of AA functionalization on NDs. All functionalized NDs (fNDs) showed averageparticle size $<200 \mathrm{~nm}$ and zeta potential of $>+20 \mathrm{mV}$, thus forming stable colloidal dispersions. ${ }^{1} \mathrm{fNDs}$ complexed with siRNA at ratios 20:1 to 50:1. (Table1) Cellular internalization of lysine-NDs in a dose dependent fashion was indicated by the increase in intracellular complexity. Clathrin-mediated-endocytosis and macropinocytosis were the mechanisms involved. ${ }^{2}$ Lysine-NDs delivered fluorescent-siRNA (3-fold increase in fluorescence for diamoplex-treated-cells (20\%) vs. untreated-cells (5\%)). ${ }^{1}$ Unlike many of the lipid-based gene carriers showing decreased transfection with serum, the protein corona on lysine-NDs facilitated the cellular uptake of diamoplexes (20\% in the presence of serum vs. $14 \%$ in the absence of serum). STXM map revealed distribution in the cytoplasm, with spectrum showing signature ND peak overlapped with cellular proteins. ${ }^{1}$ The transfection efficiency of lysine-NDs was modest due to entrapment in endo-lysosomal compartments, leading to enzymatic degradation of siRNA (major limiting step). Thus, lysyl-histidine NDs were designed (Figure1B\&C) to facilitate endosomal escape of diamoplexes. Lysylhistidine-NDs improved the gene transfection efficiency of the system (Table1). Conclusion: This study is first to demonstrate that covalent functionalization of NDs with AAs capacitate them to act as biocompatible gene carriers.

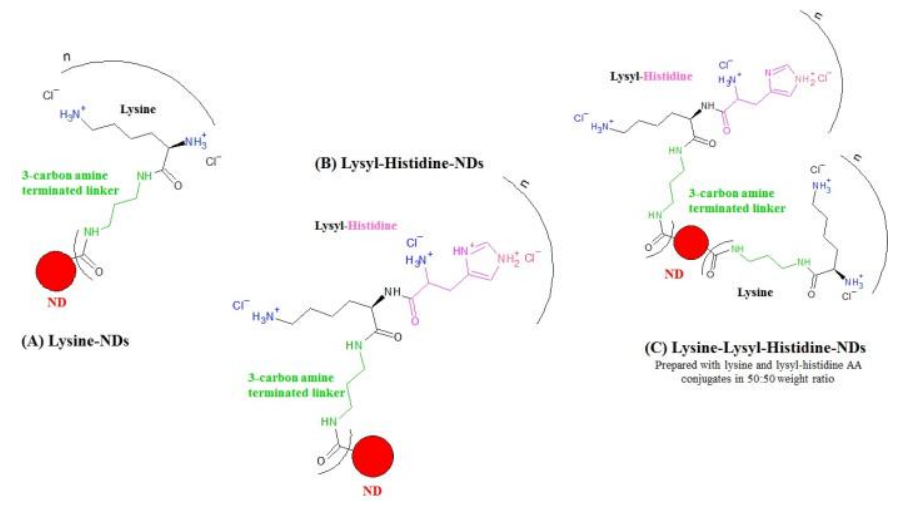

\title{
Sustained Release of Triamcinolone Hexacetonide from Poly $(\delta$-valerolactone-co-allyl- $\delta$-valerolactone $)$ Cross-linked Microparticles: A Potential Osteoarthritis Treatment
}

\author{
Zeqing Bao,,${ }^{1}$ Jack Bufton, ${ }^{2}$ Pauric Bannigan, ${ }^{3}$ Dean Aguiar, ${ }^{4}$ Christine Allen, ${ }^{5}$
}

${ }^{1} \mathrm{PhD}$ student, University of Toronto, Canada; ${ }^{2} \mathrm{PhD}$ Student, University of Toronto, Toronto, Canada; ${ }^{3} \mathrm{Post}$ Doctoral Fellow, University of Toronto, Canada; ${ }^{4}$ Chief Scientific Officer, Pendant BioSciences Inc., Canada; ${ }^{5}$ Professor, University of Toronto, Toronto, Canada

Purpose: Osteoarthritis (OA) is a degenerative joint disease affecting almost $4 \%$ of the people worldwide. Triamcinolone acetonide (TAA) and triamcinolone hexacetonide (TAH) are two corticosteroids commonly prescribed (i.e., as micronized drug suspensions) to help manage pain and inflammation associated with OA. Recently TAA has been formulated into long-acting poly(lactide-co-glycolide) (PLGA) microparticles (MPs) (i.e., Zilretta ${ }^{\mathrm{TM}}$ ), in order to reduce adverse effects (i.e., cardiovascular and metabolic), and to afford less frequent administration [1]. TAH is a prodrug of TAA and has been shown to exhibit longer joint residence time. TAH is also more hydrophobic than TAA, and this can make it challenging to formulate into MPs using existing polymeric platforms. Our group recently developed a novel, biocompatible, cross- 
linkable copolymer, $\operatorname{poly}(\delta$-valerolactone-co-allyl- $\delta$-valerolactone) (PVL-co-PAVL) [2]. Here we show this material can be used to prepare TAH and TAA loaded MPs with a high drug loading capacity (DLC) and sustained release in vitro. Methods: VL-co-PAVL MPs were prepared, and loaded with TAA or TAH using the previously described post-loading methodology [2]. MP size distribution and drug to material ratios were then evaluated using light microscopy and HPLC, respectively. In vitro drug release was assessed in PBS buffer (with 0.5\% (w/v) SDS) [2]. Results: High DLCs were achieved at $40.0 \pm 0.5$ and $37.0 \pm 3.3 \mathrm{wt} \%$ for TAA and TAH. TAA released over a period of 13 days, while TAH released over 30 days (Figure 1). Additional in vitro release studies in more bio-relevant media (i.e., artificial synovial fluid) are ongoing versus a formulation comparable to the commercially available Zilretta ${ }^{\mathrm{TM}}$. Conclusions: TAA and TAH were successfully loaded into PVL-co-PAVL MPs with high DLCs. Sustained TAA release was achieved in PBS media, and TAH had a more prolonged duration of release in this media. This suggests that TAH loaded PVL-co-PAVL MPs have potential as a long-acting injectable formulation for OA.

\section{Synergistic PARP inhibitor combinations for the treatment of high grade serous ovarian cancer}

\section{Lucy Wang, ${ }^{1}$ Lubabah Ahmed, ${ }^{1}$ Christine Allen, ${ }^{2}$}

${ }^{1}$ Graduate Student, ${ }^{2}$ Professor, University of Toronto, Toronto, Canada

Purpose: Despite recent advancements in molecular therapy (i.e. poly ADP ribose polymerase or PARP inhibitors/PARPi), ovarian cancer (OC) continues to have the poorest 5 -year survival rate amongst all gynecological cancers. While PARP inhibition can delay the onset of remission, the majority of OC cases ultimately result in relapse [1]. To combat resistance, the use of synergistic drug combinations is a promising area for investigation. Herein we describe the in vitro screening for combinations of the PARPi niraparib (NIRB) and doxorubicin (DOX) in a panel of OC cell lines. Methods: OC cell lines OVCAR8, HEYA8, PEO1, and PEO4 were seeded into 96-well plates and allowed to adhere for 12-hours prior to addition of NIRB and DOX as both monotherapy and in combination at relative molar ratios. Cells were incubated in the treatment for 72-hours prior to cytotoxicity analysis by acid phosphatase assay. To measure synergy, combination index (CI) values were determined using the Chou and Talalay method and CompuSyn software. Results: The NIRB and DOX combination showed a general trend towards additivity/synergy in the platinum sensitive OVCAR8, HEYA8, and PEO1 cell lines while displaying antagonism in the platinum resistant PEO4 cell line. Given that the PEO1 and PEO4 cell lines are a platinum sensitive/resistant pair respectively, comparison of CI values from the PEO1 versus PEO4 cell lines can provide insight into combination suitability as a second-line treatment for refractory OC. Marked antagonism in PEO4 despite synergy in PEO1 suggests that the NIRB and DOX combination could be effective as first-line but not second-line therapy. Conclusion: Combination NIRB and DOX, though synergistic in platinum-sensitive cell lines, shows consistent antagonism in a platinum-resistant cell line. These findings are in alignment with previously reported data on OLP and DOX, suggesting that DOX and PARPi combinations could be unsuitable for patients with refractory OC [2].

A)

\begin{tabular}{|c|c|c|c|c|c|c|c|c|c|}
\multicolumn{1}{c|}{ NIRB:DOX Fa=0.5 } \\
\cline { 2 - 11 } \multicolumn{1}{c|}{} & $20: 1$ & $10: 1$ & $5: 1$ & $2: 1$ & $1: 1$ & $1: 2$ & $1: 5$ & $1: 10$ & $1: 20$ \\
\hline OVCAR8 & 0.95 & 0.79 & 1.08 & 0.99 & 0.89 & 0.89 & 1.06 & 1.04 & 0.94 \\
\hline HEYA8 & 0.92 & 0.75 & 0.85 & 0.96 & 0.82 & 0.95 & 1.09 & 1.05 & 0.88 \\
\hline PEO1 & 1.01 & 0.82 & 0.96 & 0.96 & 0.93 & 1.03 & 0.79 & 0.87 & 0.81 \\
\hline PEO4 & 1.21 & 0.85 & 1.00 & 0.90 & 0.97 & 1.16 & 1.09 & 1.18 & 1.04 \\
\hline
\end{tabular}

B)

\begin{tabular}{|c|c|c|c|c|c|c|c|c|c|}
\multicolumn{1}{c|}{} & $20: 1$ & $10: 1$ & $5: 1$ & $2: 1$ & $1: 1$ & $1: 2$ & $1: 5$ & $1: 10$ & $1: 20$ \\
\hline OVCAR8 & 0.97 & 0.90 & 1.01 & 1.09 & 0.91 & 0.99 & 0.99 & 0.97 & 0.96 \\
\hline HEYA8 & 0.87 & 0.89 & 0.92 & 0.99 & 0.89 & 1.07 & 1.03 & 1.00 & 0.93 \\
\hline PEO1 & 0.89 & 0.93 & 0.94 & 0.93 & 0.96 & 1.01 & 0.92 & 0.96 & 0.89 \\
\hline PEO4 & 1.27 & 1.08 & 1.08 & 1.06 & 1.13 & 1.18 & 1.15 & 1.14 & 0.99 \\
\hline
\end{tabular}




\title{
Vinorelbine Loaded Thermosensitive Liposomes Improve Efficacy in Murine Xenograft Model of Rhabdomyosarcoma
}

\author{
Maximilian Regenold, ${ }^{1}$ James Evans,${ }^{2}$ Lucy Wang, ${ }^{3}$ Heiko Heerklotz, ${ }^{4}$ Christine Allen, ${ }^{5}$
}

${ }^{1} \mathrm{PhD}$ Student, University of Toronto, Canada; ${ }^{2}$ Project Manager, University of Toronto, Canada; ${ }^{3} \mathrm{Graduate}$ Student, University of Toronto, Toronto, Canada; ${ }^{4}$ Professor, University of Freiburg, Canada; ${ }^{5}$ Professor, University of Toronto, Toronto, Canada

Purpose: Thermosensitive liposomes (TSL) offer a path to overcome limitations associated with conventional liposomal drug delivery, heterogenous drug uptake and distribution, and limited drug release at the target site. Many drugs could benefit from localized and targeted delivery, as this can afford significant improvements in therapeutic index. Heat-triggered drug delivery of chemotherapies via TSL has recently faced a significant setback with the announcement of the failure of Thermodox ${ }^{\circledR}$ plus radiofrequency ablation in Phase III clinical trials. However, a plethora of preclinical research does highlight the potential of TSLs in combination with mild hyperthermia (HT). We previously developed a TSL formulation of vinorelbine (VRL), which rapid-releases drug at HT temperature. Here, we present studies evaluating this formulation's efficacy, in a murine xenograft model of rhabdomyosarcoma (RMS). Methods: Rapid-releasing TSLs were loaded with VRL (ThermoVRL) at different drug-to-lipid (D/L) ratios as previously described [1]. 35 SCID mice with subcutaneous Rh30 tumors were treated once per week for three treatments. Animals received either free VRL (in PBS) or ThermoVRL at a dose of 10 or $15 \mathrm{mg} / \mathrm{kg}$ via tail vein injection ( $\mathrm{n}=5 /$ group) with or without the addition of localized HT (i.e., $42.5^{\circ} \mathrm{C}$, 25mins). Results: ThermoVRL plus HT significantly improved the median survival time by a factor of 3.3 compared to free drug plus HT $(\mathrm{p}=0.004)$. No significant difference in median survival time was found between animals treated with ThermoVRL at different D/L ratios. Conclusion: This first in vivo evaluation of the performance of ThermoVRL, in combination with HT, demonstrates the advantage of heat-triggered, localized chemotherapy over the systemic administration of free drug. This drug-delivery platform can significantly improve treatment efficacy while offering a similar or improved toxicity profile.

\section{Dry-powder, nanocomposite microparticle vaccine}

$\underline{\text { Kan Kaneko, }},{ }^{1}$ Douglas De Figueiredo, ${ }^{2}$ Tasson Rodrigues, ${ }^{3}$ Daniela Ferreira, ${ }^{4}$ Carla Solorzano-Gonzalez, ${ }^{5}$ Ronan MacLoughlin, ${ }^{6}$ Eliane Miyaji, ${ }^{2}$ Viviane Maimoni Gonçalves, ${ }^{2}$ Imran Saleem, ${ }^{7}$

${ }^{1}$ Postdoctoral Research Fellow, University of Toronto, Canada; ${ }^{2}$ Researcher, Instituto Butantan, Canada ${ }^{3} \mathrm{PhD}$ student, Instituto Butantan, Canada; ${ }^{4}$ Professor, Liverpool School of Tropical Medicine, Canada; ${ }^{5}$ Postdoctoral Research Fellow, Liverpool School of Tropical Medicine, Canada; ${ }^{6} \mathrm{Head}$ of Respiratory Sciences in Aerogen, Aerogen Ltd, Canada; ${ }^{7}$ Professor, Liverpool John Moores University, Canada

Purpose: Vaccines remain the most effective form of prevention against infections. Dry powder vaccines have the potential to address numerous obstacles for conventional vaccines, such as cold-chain requirements and the possibility for direct inhalation. Despite the convenience of a dry powder formulation, the induction of sufficient immune response and subsequent protection must be satisfied as an effective vaccine. In order to implement the appropriate immunogenicity, microparticles that are directly inhaled or reconstituted for nebulization or intranasal administration, can be made to incorporate immunostimulatory nanoparticles (NPs) to enhance the immune response. This study sought to evaluate the potential of a nanocomposite microparticle (NCMP) vaccine for protection against Streptococcus pneumoniae. Methods: Polymeric NPs with a model antigen (PspA) and adjuvant, were produced by the emulsion method, and subsequently spray dried with excipients to form NCMPs. In vitro immunogenicity of NPs were evaluated 
by incubation of JAWS II cells with NPs over 24 hours and measuring CD40 and CD86 surface markers by flow cytometry. In vivo efficacy was determined through intranasal administration of the reconstituted NCMPs to BALB/c mice and measuring subsequent serum antigen-specific IgG levels, followed by infection with a model pathogen to evaluate protection. Results: Various permutations of the nanoparticle components exhibited differing NP characteristics and immunogenicity in vitro. The spray dried formulation of the optimized NPs induced high serum antigen specific IgG antibody levels of vaccinated mice, compared to groups with subcutaneous antigen administration $(\mathrm{p}<0.01)$. The NCMP vaccinated group also exhibited the highest survival, 2 weeks after challenge with the model pathogen. Conclusion: NCMPs were shown to exhibit immunogenicity and subsequent induction of antigen-specific IgG antibodies and protective immunity. These results support the feasibility of the respiratory NCMP platform.

\title{
Development of DNA supramolecules utilizing rolling circle amplification and hydrophobic interaction for efficient immunotherapy
}

\author{
$\underline{\text { Koichi Ito }},{ }^{1}$ Mutsumi Kariya, ${ }^{2}$ Yuki Takahashi, ${ }^{3}$ Yoshinobu Takakura, ${ }^{4}$ \\ ${ }^{1}$ graduate student, Graduate School of Pharmaceutical Sciences, Kyoto University, Canada \\ ${ }^{2}$ student, Graduate School of Pharmaceutical Sciences, Kyoto University, Canada \\ ${ }^{3}$ associate professor, Graduate School of Pharmaceutical Sciences, Kyoto University, Canada \\ ${ }^{4}$ professor, Graduate School of Pharmaceutical Sciences, Kyoto University, Canada
}

Purpose: Unmethylated cytosine-phosphate-guanine ( $\mathrm{CpG})$ DNA stimulates mammalian immune cells through recognition by Toll-like receptor 9 (TLR9). CpG DNA is expected to be applied for immunotherapy for diseases such as cancer and allergic disease. However, challenges such as low stability against DNase and low uptake efficiency by immune cells should be overcome for the application of $\mathrm{CpG}$ DNA. In order to overcome these challenges, we developed DNA supramolecules consisting of cholesterolmodified DNA (chol-DNA) and long single-stranded DNA (lss-DNA) prepared by rolling circle amplification (RCA). RCA products that contain repeats of $\mathrm{CpG}$ motifs were annealed with complementary chol-DNA to form the DNA supramolecules through hydrophobic interaction under aqueous condition. Methods: Formation of DNA supramolecules was confirmed by gel shift assay and transmission electron microscopy (TEM). Stability against DNase was investigated by incubating with fetal bovine serum. Cy5labeled DNA supramolecules were used to evaluate its cellular uptake by RAW264.7 cells via flow cytometry. The release of TNF- $\alpha$ by RAW264.7 was measured by ELISA to estimate immunostimulatory activity of the DNA. Results: The change of appearance in gel shift assay was confirmed by mixing cholDNA and lss-DNA, and TEM images revealed the existence of the supramolecules of which size was approximately $10 \mu \mathrm{m}$. Formation of DNA supramolecules increased stability against serum DNase compared with lss-DNA. The uptake by RAW264.7 was comparable between DNA supramolecules and lssDNA. RAW264.7 cells added with the DNA supramolecules released more TNF- $\alpha$ than that added with lssDNA did. Conclusion: We developed DNA supramolecules which show high stability against DNase and efficiently stimulate immune cells. The developed DNA supramolecules can be an effective strategy for the application of CpG DNA in immunotherapy.

\section{Nano-Encapsulation of Novel Inhibitors of ERCC1-XPF For Targeted Sensitization of Colorectal Cancer to Platinum-Based Chemotherapeutics}

Parnian Mehinrad, ${ }^{1}$ Sams Sadat, ${ }^{2}$ Michael Weinfeld, ${ }^{3}$ Feridoun Karimi-Busheri, ${ }^{4}$ Frederick G West, ${ }^{5}$ Afsaneh Lavasanifar, ${ }^{6}$ 
${ }^{1}$ Graduate student, Faculty of Pharmacy and Pharmaceutical Sciences, University of Alberta, Edmonton, $\mathrm{AB}$, Canada, Edmonton, Canada; ${ }^{2} \mathrm{PhD}$ Candidate, Faculty of Pharmacy and Pharmaceutical Sciences, University of Alberta, Edmonton, AB, Canada, Edmonton, Canada; ${ }^{3}$ Professor, Department of Oncology, Faculty of Medicine and Dentistry, University of Alberta, Edmonton, AB, Canada, Edmonton, Canada; ${ }^{4}$ Research Associate, Department of Oncology, Faculty of Medicine and Dentistry, University of Alberta, Edmonton, AB, Canada, Canada; ${ }^{5}$ Professor, Faculty of Science, Chemistry, University of Alberta, Canada; ${ }^{6}$ Professor, Faculty of Pharmacy and Pharmaceutical Sciences, University of Alberta, Edmonton, AB, Canada, Edmonton, Canada

Purpose: ERCC1-XPF nuclease is a hetero-dimeric enzyme that plays a role in nucleotide excision repair, double-strand break repair, and inter-strand crosslink repair. Cells with disabled ERCC1-XPF are particularly sensitive to DNA damage by platinum-based drugs. Our research team has developed a novel potent inhibitor of ERCC1-XPF heterodimerization, namely A4(IC50 $0.33 \pm 0.12 \mathrm{nM}$; KD $100 \pm$ $5 \mathrm{nM}$ )[1]Systemic inhibition of ERCC1-XPF can lead to sensitization of normal cells, as well as cancer cells, to the DNA damaging chemotherapeutics leading to detrimental side effects. For this reason, we propose to develop a nano-delivery system for the encapsulation of A4 to provide tumor-targeted delivery and investigate the effects of free and nano-encapsulated A4 in sensitization of colorectal cancer cells to Carboplatin. Methods: Three different self associating block copolymers, i.e., poly(ethylene oxide)-poly $(\alpha-$ benzyl carboxylate- $\varepsilon$-caprolactone)(PEO-PBCL) PEO-poly( $\varepsilon$-caprolactone)(PEO-PCL), and PEO-poly(D, L-Lactide)(PEO-PDLA) were used for the encapsulation of A4. Developed nano-delivery systems were characterized for their average diameter, A4 encapsulation, and in vitro release. The cytotoxicity of A4 as free and encapsulated in PEO-PBCL nanoparticles (NP)s was assessed against HCT116, SW620, and HT29 cells alone and upon co-treatment with carboplatin using the MTT assay. Results: Among NP formulations of A4, PEO-PBCL provided the best physicochemical characteristics (average diameter of $45.19 \pm 0.32 \mathrm{~nm}$, PDI: $0.120 \pm 0.008,83.06 \pm 5.8 \%$ encapsulated efficiency; $<50 \%$ drug release in $24 \mathrm{~h}$ ). The results of the cytotoxicity study showed a significant reduction in the viability of the HCT116 and SW620 cell line following co-treatment of $0.5 \mu \mathrm{M}$ of A4 (as free and particularly $\mathrm{Np}$ form) with carboplatin $(25-100 \mu \mathrm{M}$ ). The SW620 cell line was more sensitive to the effect of A4. Conclusion: Results indicate a potential for PEO-PBCL NP formulations of A4 for chemosensitization of CRC platinum chemotherapeutic.support: Alberta Cancer foundation

\section{BCS-based biowaiver using biphasic dissolution test}

\section{Daniela Amaral Silva, ${ }^{1}$ Katherine Curo Melo, ${ }^{2}$ Neal Davies, ${ }^{3}$ Nadia Bou-Chacra, ${ }^{4}$ Humberto Ferraz, ${ }^{5}$ Raimar Löbenberg, ${ }^{6}$}

${ }^{1} \mathrm{PhD}$ candidate, University of Alberta, Canada; ${ }^{2} \mathrm{PhD}$ candidate, Universidade de Sao Paulo, Canada; ${ }^{3}$ Dean and Professor, University of Alberta, Canada; ${ }^{4}$ Professor, Universidade de Sao Paulo, Canada ${ }^{5}$ Dean and Professor, Universidade de Sao Paulo, Canada; ${ }^{6}$ Professor, University of Alberta, Edmonton, Canada

Purpose: Biowaivers based on BCS class can be used to establish therapeutic equivalence based on dissolution tests. Such tests are used as a surrogate to determine if two pharmaceutical equivalent products are interchangeable/bioequivalent. The objective of the present study was to compare a biphasic dissolution system with compendial methods in examining the in vitro performance of widely used drug products. We hypothesized that where in vitro equivalence was not achieved in compendial methods, the partitioning profile to the organic phase in the biphasic system could signal bioequivalence among the drug products andcomparator pharmaceutical product (CPP). Methods: Five commercial metronidazole tablets were tested in compendial Simulated Intestinal Fluid (SIF), as well as in physiological buffer capacity (5mM phosphate buffer at $\mathrm{pH} 6.8,900 \mathrm{~mL}$ ). The tablets were also tested in a biphasic dissolution system in which the aqueous layer was composed of $200 \mathrm{~mL}$ of $5 \mathrm{mM}$ phosphate buffer (pH 6.8) with $100 \mathrm{~mL}$ of $\mathrm{n}$-octanol 
on top. Results: None of the tested metronidazole products were in vitro equivalent to the CPP or to other manufacturers in compendial buffer. The tested metronidazole products followed a similar pattern than that obtained in the compendial buffer in the aqueous phase of the biphasic system. However, this was not the case for the organic phase partition profiles. All the tested products had a good correlation to the CPP, which could indicate in vitro equivalence between these products. This could potentially allow for a biowaiver application. Conclusion: The application of biphasic dissolution to highly soluble drugs might be beneficial to estimate the product's in vivo behavior.

\section{Improved in vitro - in vivo relationship applying physiologically relevant dissolution conditions}

Daniela Amaral Silva, ${ }^{1}$ Marcelo Gomes Davanço, ${ }^{2}$ Neal Davies, ${ }^{3}$ Patricia De Oliveira Carvalho, ${ }^{4}$ Raimar Löbenberg, ${ }^{5}$

${ }^{1} \mathrm{PhD}$ candidate, University of Alberta, Canada; ${ }^{2} \mathrm{PhD}$ student, Universidade São Francisco, Canada; ${ }^{3}$ Dean and Professor, University of Alberta, Canada; ${ }^{4}$ Professor, Universidade São Francisco, Canada; ${ }^{5}$ Professor, University of Alberta, Edmonton, Canada

Purpose: There are many hurdles in the development of generic formulations. In vitro biopredictive dissolution conditions together with alternative in vitro - in vivo relationship (IVIVR) approaches can be a powerful tool to support the development of such formulations. In this study, we hypothesized that the release profile of enteric coated (EC) formulations of pantoprazole in physiologically relevant bicarbonate buffer (BCB) would detect possible performance differences between test and reference formulations resulting in more accurate IVIVR results and predictability when compared to a pharmacopeial dissolution test. Methods: Dissolution tests were performed in BCB and pharmacopeial phosphate buffer for both test and reference formulations. The in vitro dissolution profiles were correlated with the human in vivo data from a failed bioequivalence study. Results: Test and reference formulations of EC pantoprazole tablets passed the USP dissolution criteria. However, they failed statistical similarity in vitro both in compendial and BCB. A better IVIVR was obtained with BCB and this buffer system was additionally more discriminative while being more physiologically relevant (Figure 1). Conclusion: Having BCB as an additional test to evaluate EC products in vitro might improve the comparison of formulations. This can derisk the development of generic $\mathrm{EC}$ formulations.

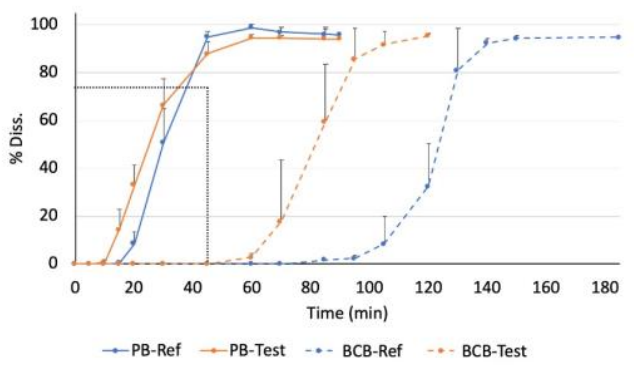

\section{Overcoming the challenge of poor efficiency in bio conjugation reactions by physical approach}

\section{Ahlem Meziadi, ${ }^{1}$}

${ }^{1}$ Institut National de la recherche scientifique INRS, Canada

The poor efficiency of PEGylation reaction in terms of number of $\mathrm{mPEG}$ chains grafted to the protein relative to the amount used for the reaction, generally implies that a large excess of mPEG is used.[1] This 
not only drives up the cost of producing a given $\mathrm{mPEG}$-protein bio conjugate, but also complicates purification. In this work, we report a novel strategy for protein PEGylation by transient heating of the solution using a simultaneously-cooled microwave (MW) reactor, promoting the interaction between mPEG and proteins. The MW reactor operates at a frequency of $2.45 \mathrm{GHz}$, and provides the ability to keep a constant bulk reaction temperatures of $\sim 25^{\circ} \mathrm{C}$ while the reaction mixture is exposed to MW irradiation[2]. The stability of several proteins within the microwave set-up was tested. For this purpose, a solution of each desired protein was prepared in phosphate buffer and placed in the reaction vessel. Then, the solution was exposed to MWs at different powers for a fixed time. After microwaving, the intactness of the proteins was assayed via the enzymatic activity and conformational studies. No damage to the proteins was observed under these conditions.[3] Having validated that the proteins were not deleteriously affected by MW irradiation, its ability to enhance PEGylation was further examined. It was found that: (i) MW PEGylation could operate at a low reactant ratio while the conventional process needs a much higher ratio; (ii) higher reaction rate in MW, (iii) shorter chains of PEG gave a better result in MW, and (iv) conventional PEGylation and exposed reactions to MW follow different reaction orders. Overall, this work demonstrated that MW irradiation at room temperature $\left(\sim 25^{\circ} \mathrm{C}\right)$ exhibited promising results as a new tool for increasing the efficiency of PEGylation reactions and may have application potentials in various conjugations of bio macromolecules at lower cost and shorter time.
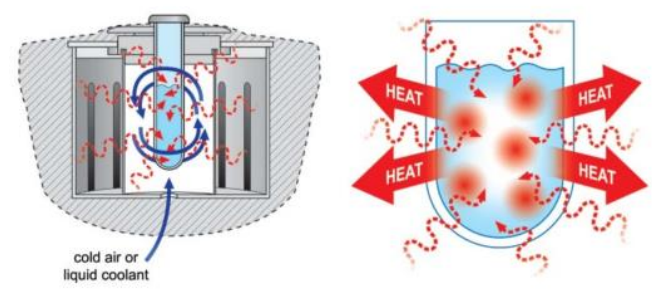

\section{Development of a digestion-resistant self-nanoemulsifying drug delivery system for the oral delivery of cannabidiol}

Lie Yun Kok, ${ }^{1}$ Pauric Bannigan, ${ }^{2}$ Forugh Sanaee,${ }^{3}$ James Evans, ${ }^{4}$ Lubabah Ahmed, ${ }^{1}$ Maximilian Regenold,${ }^{5}$ Christine Allen, ${ }^{6}$

${ }^{1}$ Graduate Student, ${ }^{2}$ Post Doctoral Fellow, ${ }^{3}$ Postdoctoral fellow, ${ }^{4}$ Project Manager, ${ }^{5} \mathrm{PhD}$ Student, ${ }^{6}$ Professor, University of Toronto, Toronto, Canada

Purpose: Cannabidiol (CBD) is a phytocannabinoid that has shown potential in the treatment of epilepsy and inflammation ${ }^{1}$. CBD exhibits poor oral bioavailability due to its lipophilicity and extensive metabolism in the liver. One strategy to improve the solubility of CBD is to use self-nanoemulsifying drug delivery systems (SNEDDS). SNEDDS are mixtures of oil, surfactants, and co-solvents that can disperse in gastrointestinal fluids to form oil-in-water nanoemulsions. However, digestion of these formulations may result in drug precipitation prior to absorption ${ }^{2}$. Here, a SNEDDS formulation was developed using a digestion-resistant surfactant. The CBD-SNEDDS formulation was evaluated in vivo and compared to a commonly used oil formulation of CBD. Method: Excipients were selected based on solubility studies of CBD. Lead formulations were loaded with CBD $(10 \% \mathrm{w} / \mathrm{w})$ and evaluated in terms of droplet size and solubilization following dispersion in biorelevant media. In vitro digestion assays were also conducted on these formulations. Selected formulations were evaluated in vivo, with a single dose of CBD (20 mg/kg) administered orally. Pharmacokinetic parameters were calculated using the non-compartmental model. Results: Based on CBD solubility, an ether-based surfactant, medium-chain triglyceride (MCT) oil, and a co-solvent were selected for the SNEDDS formulations. Three of the lead formulations formed droplet sizes 
under $200 \mathrm{~nm}$ in biorelevant media. Minimal drug precipitation was observed during in vitro digestion of these formulations. Oral administration of the various CBD-SNEDDS formulations increased the area under the curve values of CBD by 2.0 to 2.5 -fold, compared to the control (Figure 1). Conclusion: Overall, administration of the CBD-SNEDDS formulations resulted in greater in vivo exposure compared to the control (CBD in oil carrier). These results suggest that advanced formulations have the potential to enhance the oral bioavailability of CBD.

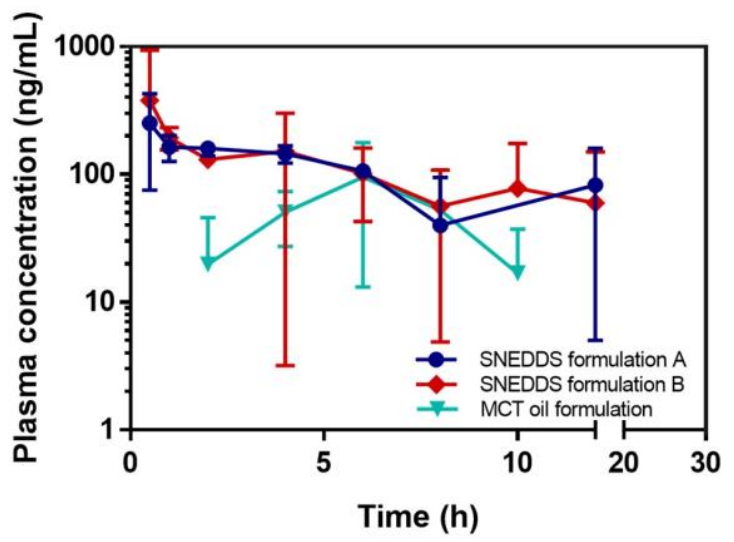

\section{Investigation of the novel cannabinoids-loaded emulsifying system for improved transdermal delivery and stability}

Chulhun Park, ${ }^{1}$ Jieyu Zuo, ${ }^{2}$ Vijay Somayaji, ${ }^{3}$ Raimar Löbenberg, ${ }^{4}$

${ }^{1}$ Postdoctoral fellow, ${ }^{2}$ Postdoctoral fellow, ${ }^{3}$ Manager, DDIC, ${ }^{4}$ Professor, University of Alberta, Edmonton, Canada

Purposes: The potential therapeutic usage of cannabinoids has been challenging due to their low solubility. Medicinal cannabis products can be designed for oral administration with oil-based types of formulations. This study aimed to develop a stable cannabinoid-loaded microemulsion (ME) containing tetrahydrocannabinolic acid (THCA), and cannabidiolic acid (CBDA). Methods: Formulations were assessed for selected oil and surfactant vehicles. The pseudo-ternary phase $\mathrm{Capryol}^{\circledR} \mathrm{90/ \textrm {S } _ { \text { mix } }}$ combination with ethanol (Tween ${ }^{\circledR} 80$, Solutol ${ }^{\circledR}$ HS15, Procetyl ${ }^{\circledR}$ AWS, and Cremophor ${ }^{\circledR}$ RH40)/Water systems were investigated. Morphology and structure of MEs were determined by electrical conductivity and transmission electron microscope (TEM). The cannabinoids-loaded P2 ME (Capryol ${ }^{\circledR} 90$ (5.0\%), S $_{\text {mix }}$ (Procetyl ${ }^{\circledR}$ AWS: Ethanol=2:1) (45.0\%), and water (50.0\%)) showed stable o/w phase behavior. The effects of $\mathrm{pH}$ on the permeation of cannabinoids were investigated by an aqueous buffer system. Results: After loading cannabinoids with $1.0 \%$ w/w, each P2 ME showed the mean droplet size of $89.62 \pm 7.89 \mathrm{~nm}$ (THCA), $84.72 \pm 6.32 \mathrm{~nm}(\mathrm{CBDA})$ Based on the specific $\mathrm{pH}$ value of cannabinoids-loaded P2 ME, THCA and CBDA-loaded $\mathrm{P} 2 \mathrm{ME}$ respectively exhibited the highest permeation profiles at $\mathrm{pH} 4.92$ and $\mathrm{pH} 5.25$. After storing for 3 months at ambient and $4^{\circ} \mathrm{C}$ conditions, the stability of cannabinoids in P2 MEs maintained their assay over 95\% compared to cannabinoids in ethanol extraction. Conclusion: Capryol 90-based P2 ME could be a versatile option for solubilizing and stabilizing lipophilic drugs like cannabinoids from herbal extracts and be useful for transdermal delivery. Funding: This research was supported by a grant from the Drug Development Innovation Center and supported by Basic Science Research Program through the National Research Foundation of Korea (NRF) funded by the Ministry of Education (NRF2020R1A6A3A03037334). 


\title{
Utilization of Click Chemistry to Study the Interplay of Linker Chemistry and PEG Molecular Weight on the Pharmacokinetics and Antitumor Efficacy of Gambogic Acid Nanoparticles
}

\author{
Anne Nguyen, ${ }^{1}$ Shyh-Dar Li, ${ }^{2}$ \\ ${ }^{1} \mathrm{PhD}$ Candidate, ${ }^{2}$ Professor, UBC Pharmaceutical Sciences, Vancouver, Canada
}

Purpose: Prodrugs are designed to improve pharmaceutical properties of potent compounds and represent a central approach in drug development. The success of the prodrug strategy relies on incorporation of a reversible linkage facilitating controlled release of the parent drug. While prodrug approaches enhance pharmacokinetic properties over their parent drug, small molecule prodrugs still face challenges in absorption, distribution, metabolism, elimination, and toxicity (ADMET). Conjugating a drug to a carrier molecule such as a polymer can create a prodrug that self-assembles into nanoparticles. These nanoparticles display prolonged blood circulation and passive targeting ability. Furthermore, the drug release can be tailored using a variety of linkers between the parent drug and the carrier molecule. Methods: Gambogic Acid (GA) was modified using a previously developed synthetic platform to produce six different polymerdrug conjugates that were formulated into nanoparticles (NPs). These six NPs vary in linker (ester or amide), and PEG molecular weight $(550,2000,5000)$. Physiochemical properties such as size, polydispersity index (PDI) and critical micellar concentration (CMC) were collected. In vitro drug release studies were performed in plasma. The pharmacokinetics of these NPs were determined in mice. In addition, the efficacy of GA-NPs was tested in an in vivo tumor model. Results: Nanoparticles with diameter of 100-200 nm were formed with all six polymer-drug conjugates. The linker and PEG molecular weight of the polymer-drug conjugates affected drug release, pharmacokinetics, and anti-tumor efficacy of the resulting six GA-NPs. Conclusion: The linker and PEG molecular weight of PEG-drug conjugates plays a significant role in drug release, pharmacokinetics, and anti-tumor efficacy. The simple synthetic platform used in this study proves to be a valuable tool in efficiently designing prodrug-based nanoparticles.

\section{Reduced primaquine hemolytic toxicity of red blood cells and improved liver delivery by phospholipid-free small unilamellar vesicles}

\author{
$\underline{\text { Nojoud AL Fayez, }}{ }^{1}$ Shyh-Dar Li, ${ }^{2}$ \\ ${ }^{1}$ Graduate student, ${ }^{2}$ Professor, University of British Columbia, Vancouver, Canada
}

Purpose: Malaria remains one of the most lethal parasitic diseases in tropical regions around the world [1]. High oxidative stress levels caused by primaquine (PQ), a standard treatment to cure and prevent liver stage malaria, can lead to serious hemolytic anemia in some populations. In addressing this challenge, we demonstrated that phospholipid-free small unilamellar vesicles (PFSUVs), composed of non-ionic surfactant and cholesterol, are able to encapsulate and deliver PQ directly to hepatocytes with limited exposure to red blood cells (RBCs). Methods: Non-ionic surfactant (TWEEN80) and cholesterol forming nano-vesicles encapsulating PQ was fabricated using microfluidic system. Ex-vivo PQ uptake in red blood cells (RBCs) and in vivo pharmacokinetics in mice was evaluated for efficient PQ delivery to the liver using PFSUVs in comparison to free drug. Additionally, the effect of oxidative stress on RBCs was illustrated by examining cell morphology in the presences of PQ and PFSUVs-PQ. Results: Optimized PFSUVs encapsulated $>95 \%$ PQ with stable drug retention in the presence of serum. PQ uptake in RBCs displayed up to 8-fold decrease in concentration when treated with PFSUVs group compared to free PQ, which consequently led to a significant change in RBC morphology. Additionally, PFSUVs selectively targeted hepatocytes when administered intravenously, and displayed an increase in liver uptake by 4.8 and 1.6-folds compared to free PQ given intravenously and orally, respectively. Conclusion: PFSUVs encapsulating PQ 
showed significant drug accumulation in liver with low RBCs exposure and toxicity. As a result, PFSUVs, exhibited a potential to improve efficacy and safety of PQ for treating liver-stage malaria.

\title{
Development of Novel In-vitro Model to Study Lymphatic Uptake of Drugs Following Oral Administration
}

Malaz Yousef, ${ }^{1}$ Neal Davies, ${ }^{2}$ Raimar Löbenberg, ${ }^{3}$

${ }^{1}$ Ph.D. candidate, ${ }^{2}$ Dean and Professor, ${ }^{3}$ Professor, Faculty of Pharmacy and Pharmaceutical Sciences, University of Alberta Edmonton, Canada

Purpose: To develop a novel in-vitro model to study the uptake of oral lymph targeted formulations using simulated lymphatic fluid in Franz cell set up. Methods: The release characteristics of the model drug (rifampicin) were investigated using the Franz cell model. Accurately measured rifampicin was added to the donor compartment containing intralipid [an emulsion representing the endogenous lymph carried species (chylomicrons)]. The receiver compartment was filled with $12 \mathrm{ml}$ of simulated lymphatic fluid ( $\mathrm{pH} 7.2$ ), maintained at $37.0 \pm 0.5^{\circ} \mathrm{C}$ and magnetically stirred at $600 \mathrm{rpm}$ throughout the experiment. The compartments were separated by a synthetic $0.22 \mu \mathrm{m}$ Polyvinylidene Fluoride (PVDF) membrane impregnated with octanol. At the time points $(0.5,1,2,3,4$ and $6 \mathrm{~h}), 0.2 \mathrm{ml}$ samples were withdrawn and extracted with methanol (extraction efficiency $92 \%$ ) before being analyzed using HPLC. Results: The salts concentration in the proposed simulated lymphatic fluid was adopted from Marques et al [1] to coincide with that of the extracellular fluid. Additionally, albumin and intralipid were used to represent the protein and the lipid components of the lymph in the percentages reported in the literature [2]. Preliminary data confirmed the solubility of the rifampicin in the intralipid and the simulated fluid and was found to be 0.7 $\mathrm{mg} / \mathrm{ml}$ and $1.31 \mathrm{mg} / \mathrm{ml}$, respectively. The higher solubility of the drug in the simulated fluid might have driven its release through the membrane in the Franz cell. Conclusion: Franz cell model might be used to mimic the translocation of drugs from the cytoplasm of the enterocytes to the lymphatic system. Besides, the use of octanol made the membrane more representative of the cellular lipid bilayer. This might open a door to better understand the in-vivo uptake of the chylomicrons associated drugs into the intestinal lymphatics without using cellular or animal model.

\section{PHARMACEUTICAL AND ANALYTICAL CHEMISTRY}

\section{Myeloperoxidase (MPO)-mediated oxidation of quetiapine and clozapine: Capability of MPO + Cl- in} cytochrome P450-like reaction on quetiapine

\author{
$\underline{\text { Md Rashid }},{ }^{1}$ Arno Siraki, ${ }^{2}$
}

${ }^{1}$ PhD Student, Faculty of Pharmacy \& Pharmaceutical Sciences, University of Alberta, Canada. Institute of Food and Radiation Biology, Bangladesh Atomic Energy Commission, Bangladesh, Edmonton, Canada ${ }^{2}$ Professor \& Director, Faculty of Pharmacy \& Pharmaceutical Sciences, University of Alberta, Edmonton, Canada

Purpose: Both quetiapine and clozapine are important atypical antipsychotic drugs but have a dangerous side-effect (agranulocytosis) that is thought to involve reactive metabolites [1-3]. A key aromatic nitrogen atom of the clozapine appears to be the reactive center that is responsible for the formation of reactive intermediates by neutrophil myeloperoxidase (MPO) [4]. However, key aromatic sulfur making quetiapine less labile for oxidation than the nitrogen in clozapine [5]. Rationale: The MPO metabolism of quetiapine is 
not well-studied, but it is likely an MPO substrate. Also, it was reported that quetiapine is metabolized by cytochrome P450 [6]. Hypothesis: Based on structural similarity to clozapine, quetiapine can be metabolized by MPO or MPO $+\mathrm{Cl}^{-}$and there is a relationship between cytochrome $\mathrm{P} 4503 \mathrm{~A}$ and $\mathrm{MPO}$ on the metabolism of quetiapine. Methods: MPO active site assay by UV-Vis spectrophotometry, MPO peroxidase activity assay, MCD chlorination activity assay, LC-MS, and EPR spectroscopy. Results: MPO Compound II accumulated in the presence of quetiapine whereas, MPO Compound II was rapidly reduced back to its native state in the presence of clozapine. Increasing quetiapine concentrations resulted in a proportional decrease in MPO chlorination, while the opposite result was found in clozapine. No change when quetiapine was oxidized, but clozapine showed change. However, hydroxylated and dealkylated MPO-catalyzed metabolites of quetiapine and some hydroxylated metabolites of clozapine were proposed from the LC-MS analyses. Clozapine oxidized GSH to $\bullet$ SG, whereas quetiapine did not produce $\bullet$ SG. Conclusions: MPO is responsible for the metabolism of quetiapine (S-oxidation and dealkylation). Although the reactive metabolites were hypothesized to be involved in drug-induced agranulocytosis, this does not appear to be a relevant mechanism for quetiapine as opposed to clozapine. Moreover, $\mathrm{MPO}+\mathrm{Cl}^{-}$is capable of cytochrome P450 3A-like reaction on quetiapine. In the future, we will compare the yield of cytochrome P450 products vs MPO. Keywords: Myeloperoxidase, Quetiapine, Clozapine, Reactive Metabolite, Agranulocytosis.

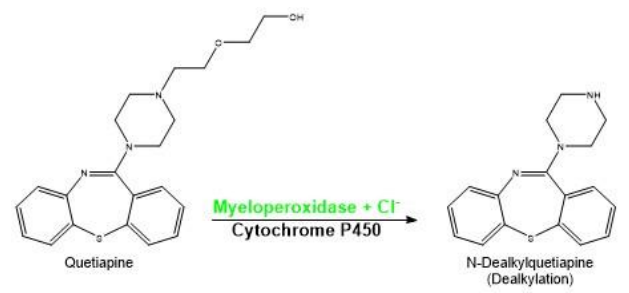

\section{Investigating a Unique Cancer-Associated Glycan on Breast Cancer Cell Derived Extracellular Vesicles}

\section{$\underline{\text { Wing Sum Tam }},{ }^{1}$ Nikki Salmond, ${ }^{2}$ Karla Williams ${ }^{3}$}

${ }^{1}$ Undergraduate Student, ${ }^{2}$ Postdoctoral Fellow, ${ }^{3}$ Assistant Professor, Faculty of Pharmaceutical Sciences, The University of British Columbia, Vancouver, Canada

Purpose: Understand the role that a unique cancer-associated glycan plays in breast cancer cell-cell communication and metastasis. Methods: Breast cancer cell lines were Western blotted for the presence of the glycan of interest. Extracellular vesicles (EV) were isolated by ultracentrifugation and characterised using nanoparticle tracking analysis (NTA) and transmission-scanning electron microscopy (T-

SEM). Results: Western blots showed that the metastatic MDA-MB-231-Lymph Node (LN) derived cell line expressed more cancer-associated glycan of interest as compared to parental non-metastatic breast cancer cell line MDA-MB-231 (231). Cancer associated glycan was enriched in EVs isolated from LN cells by ultracentrifugation at $100,000 \mathrm{x}$ g as compared to LN cell lysate. EVs expressed EV markers (CD63, CD81, ALIX, TSG101), whilst proteins indicative of EV preparation contamination (calnexin, bovine serum albumin) were largely absent. Isolated EVs imaged using T-SEM had a donut shaped morphology and were 100-200 $\mathrm{nm}$ in size. NTA showed that the EVs had an average size of $131.42 \mathrm{~nm}$ and an average concentration of 5.67E10 particles $/ \mathrm{ml}$. To further investigate the role of the cancer-associated glycan in cancer, the glycan was removed from EVs using an enzyme that specifically removes the glycan from proteins. This had no effect upon EV morphology as confirmed by T-SEM or uptake into 231 and LN cells as shown by immunofluorescence microscopy. Conclusion: EVs from LN cells were characterized by Western blot, NTA and T-SEM. The unique cancer-associated glycan was enriched on LN EVs as 
compared to cell lysate. The glycan can be removed from proteins using an enzyme without affecting the EV morphology or the uptake of EVs into recipient LN and parental 231cells. We have developed an experimental platform that will be used to study the role of this unique cancer-associated glycan in breast cancer cell-cell communication and cancer cell metastasis.

\title{
A single LC-MS/MS method to quantify cannabinoids in blood, breast milk and meconium
}

\author{
Anaëlle Monfort, ${ }^{1}$ Martin Jutras, ${ }^{2}$ Gregory Lodygesnky, ${ }^{3}$ Ema Ferreira,,${ }^{4}$ Grégoire Leclair, ${ }^{5}$
}

${ }^{1}$ Student, Université de Montréal, Canada; ${ }^{2}$ Bioanalyse and LC-MS/MS specialist, Université de Montréal, Canada; ${ }^{3}$ Professor and doctor, Université de Montréal, CHU Sainte-Justine, Canada; ${ }^{4}$ professor and pharmacist, Université de Montréal, CHU Sainte-Justine, Canada; ${ }^{5}$ professor, Université de Montréal, Canada

Purpose: Since the recent legalization of cannabis in Canada, health professionals have been counselling more women on the use of cannabis during pregnancy and lactation. However, the data available on the effects of cannabis on the brain development of newborns is scarce. Thus, we are conducting a study to determine the effects of cannabis on brain development. As part of this study, we aimed at developing analytical methods in various matrices such as meconium, breast milk, and infant blood to quantify the infant exposure to cannabis. Methods: Liquid chromatography tandem mass spectrometry (LC-MS/MS) methods were developed and validated to quantify cannabinoids in meconium, breast milk, and infant blood. Three different extraction methods were used for these complex matrices. Linearity, accuracy, precision, selectivity, matrix effect, carry-over, recovery, and stability were evaluated to validate the methods. Results: A single LC-MS/MS method was developed for every matrix to quantify THC, CBD, CBN, 11-OH-THC and 11-THCCOOH. Cannabinoids were extracted from meconium and breast milk by solid phase extraction with HLB Prime cartridges and extracted from blood by liquid-liquid extraction. Dansyl chloride was used as a derivative in blood samples to increase the sensitivity of the method as only $20 \mu \mathrm{L}$ of blood was used for the study. Calibration curves were ranging from 1.5 to $500 \mathrm{ng} / \mathrm{mL}$ in breast milk. Precision (3.1-12.3\%) and accuracy (89.5-109.6\%) of the breast milk method complied with specifications from international guidelines. The standard deviation of matrix effect and recovery was less than $15 \%$. Conclusion: These methods will allow the quantification of cannabinoids in meconium, breast milk and infant blood in order to correlate the effects of cannabis on the brain development of newborns to the exposure during pregnancy and breastfeeding. In long term, it will help to update the Canadian guidelines on the use of cannabis during pregnancy and breastfeeding.

\section{Development and partial validation of a bioanalytical assay for the detection of a novel bone- targeting parathyroid hormone conjugate using metal-free LC-MS/MS}

\section{Ben Wajda, ${ }^{1}$ Dion Brocks, ${ }^{2}$ Michael Doschak, ${ }^{3}$ Ali Aghazadeh-Habashi, ${ }^{4}$}

${ }^{1}$ Student, University of Alberta, Canada; ${ }^{2}$ Professor, University of Alberta, Canada; ${ }^{3}$ Professor, Faculty of Pharmacy \& Pharmaceutical Sciences, University of Alberta, Edmonton, Canada; ${ }^{4}$ Professor, Idaho State University, Canada

Purpose: To address shortcomings with current drug treatments for osteoporosis and improve the efficacy of human parathyroid hormone (hPTH(1-34)), we have employed a bisphosphonate-conjugation strategy by tethering $\mathrm{hPTH}(1-34)$ to a non-nitrogenated bisphosphonate (BP) moiety (BP-PTH) in order to enhance targeted drug delivery to bone. Conjugation of hPTH (1-34) to a BP imparts mineral affinity to the peptide hormone, allowing it to localize to positively-charged calcified tissues in bone. Improved drug targeting 
will lead to administration of lower therapeutic doses, therefore it is essential to develop a sensitive bioanalytical assay for future pharmacokinetic studies. Bisphosphonate groups pose many analytical and chromatographic challenges due to their high polarity and strong chelating abilities. Analysis was further complicated due to the large molecular weight (5869.9 Da) of the conjugate, requiring high ionization energy for fragmentation. Methods: A combination of protein precipitation and solid phase extraction was used for sample preparation, allowing for concentrated samples without the need of evaporation. Chromatographic separation was performed on a reverse-phase bioinert PS-C18 column with a total run time of $10.50 \mathrm{~min}$. Metal-free LC-MS/MS was employed to avoid undesirable phosphate group binding to metal surfaces in the LC-MS/MS flow path and reduce chelation. Results: The calibration curve was linear $\left(\mathrm{r}^{2}=0.996\right)$ in the range of $5 \mathrm{ng} / \mathrm{mL}$ to $25 \mathrm{ng} / \mathrm{mL}$, the lower limit of quantification was $2.5 \mathrm{ng} / \mathrm{mL}$. The intraand inter-assay precision over two days (percent coefficient of variation) calculated from quality control samples was less than 10\%, and the mean accuracy (percent deviation from nominal) for all samples was between $102.22 \%$. Conclusions: Despite achieving satisfactory validation parameters, the chosen analytical column proved to have a short column lifetime before becoming irreversibly damaged. The method will be re-optimized with a more appropriate analytical column in the future. To our knowledge, this is the first reported method for the detection and quantification of BP-conjugated peptides in a biological matrix.

\title{
An HPLC method to determine the stability of Lidocaine and Ketoprofen compounded individually, and in combination, with topical gel formulations.
}

\author{
Shadab Alam, ${ }^{1}$ Miguel H. Rueda, ${ }^{2}$ Leanne Hahn, ${ }^{3}$ Rakesh Bhat, ${ }^{4}$ Michael Doschak, ${ }^{5}$ \\ ${ }^{1}$ Industrial Postdoctoral Fellow, University of Alberta, Edmonton, Canada; ${ }^{2}$ Postdoctoral Fellow, University \\ of Alberta, Edmonton, Canada; ${ }^{3}$ Trainee, Applied Pharmaceutical Innovation (API), Edmonton, Canada; \\ ${ }^{4}$ Director, Laboratory Science, Applied Pharmaceutical Innovation (API), Edmonton, Canada; ${ }^{5}$ Professor, \\ Faculty of Pharmacy \& Pharmaceutical Sciences, University of Alberta, Edmonton, Canada
}

Purpose: Pluronic lecithin organogel (or PLO gel) cream base is used in compounding pharmacies to formulate various drug combinations, to meet patient-specific needs. Regulatory requirements for compounding pharmacies now include documented stability of any compounded preparations under specified mixing conditions. As such, the purpose of this project is to establish extraction procedures and develop high-performance liquid chromatography (HPLC) assay protocols for a variety of small molecule analgesic drugs, in order to confidently assign the shelf-life to respective preparations of compounded of active pharmaceutical ingredients under usual storage conditions. Here we report a common extraction and analytical HPLC method to determine the stability of Lidocaine and Ketoprofen in PLO gel compounded separately and in combination at different time period. Method: To extract the drug molecules 1:1 mixture of acetonitrile and water showed the best recovery and no solvent peak during the HPLC run. Gemini ${ }^{\circledR} 5 \mu$, C18 column were used to resolve the drug and quantify the recovery. Result: Extraction of active drug from all the three combinations (lidocaine + PLO, ketoprofen + PLO and lidocaine + ketoprofen + PLO) showed $100 \%$ recovery with less than 5\% RSD during the course of study. Conclusion: Consistent absolute recovery of these drugs revealed their ongoing activity within the formulation for the study duration of 12 months. The developed method is suitable to assay Lidocaine and Ketoprofen with PLO gel and may prove useful for other dosage forms, such as transdermal patches, ointments, etc.

\section{Population pharmacokinetic model-informed dosing of vancomycin in neonates}

\section{Erin Chung, ${ }^{1}$ Winnie Seto, ${ }^{2}$}

${ }^{1} \mathrm{PhD}$ Candidate, Graduate Department of Pharmaceutical Sciences, University of Toronto; Department of Pharmacy, The Hospital for Sick Children, Canada; ${ }^{2}$ Senior Pharmacy Manager, Department of Pharmacy, 
The Hospital for Sick Children; Graduate Department of Pharmaceutical Sciences, University of Toronto; Child Health Evaluative Services, The SickKids Research Institute; Institute of Health Policy, Management and Evaluation, University of Toronto, Canada

Purpose: Neonatal sepsis is an invasive bloodstream infection occurring in newborns, commonly caused by coagulase-negative staphylococci (CoNS) in the neonatal intensive care unit (NICU). Vancomycin is the drug of choice to treat CoNS sepsis, yet dosing remains a challenge in neonates due to significant pharmacokinetic variability. Therefore, we aimed to develop a population pharmacokinetic (popPK) model for vancomycin in neonates in order to derive more optimal initial vancomycin dosing to improve target attainment. Methods: This was a single-centre, retrospective study that included neonates admitted to NICU receiving intravenous vancomycin. PopPK analyses were conducted using nonlinear mixed effects model (NONMEM). The final popPK model was then used to simulate dosing using Monte Carlo simulations. Therapeutic ranges used in the analyses included area-under-the-concentration-time-curve of $400-600 \mathrm{mg} * \mathrm{~h} / \mathrm{L}$ or trough concentration of $10-15 \mathrm{mg} / \mathrm{L}$ for central nervous system (CNS) infections or 5$12 \mathrm{mg} / \mathrm{L}$ for other infections. Results: Out of 201 vancomycin courses, initial vancomycin dosing resulted in $67-80 \%$ of vancomycin trough concentrations outside target range for CNS infections $(10-15 \mathrm{mg} / \mathrm{L})$ or 29-43\% outside target range for other infections (5-12 mg/L). A one-compartment model best described the observed data from 181 neonates with 296 detectable vancomycin concentrations. The mean clearance was $0.11 \pm 0.03 \mathrm{~L} / \mathrm{kg} / \mathrm{h}$ and volume of distribution $(\mathrm{V})$ was $1.02 \pm 0.08 \mathrm{~L} / \mathrm{kg}$. Bodyweight, postmenstrual age (PMA), and serum creatinine ( $\mathrm{SCr}$ ) were significant covariates associated with clearance $(\mathrm{p}<0.001)$ and bodyweight was a significant covariate associated with $\mathrm{V}(\mathrm{p}=0.009)$. Based on simulations with the popPK model, a new dosing guideline for vancomycin in neonates was derived with stratification by PMA and SCr, which improved probability of target attainment. Conclusion: Neonates are at risk of not achieving target vancomycin concentrations, which increase their risk of ineffective therapy or nephrotoxicity. We suggest implementing a popPK model-informed vancomycin dosing guideline in neonates with therapeutic drug monitoring to improve target attainment.

\section{Analysis of the relationship between age and $\mathrm{VWF}$ in hemophilia A: the precision of using age as a proxy for vWF in FVIII population pharmacokinetic models for estimating PK}

$\underline{\text { Svetlana Litchmanova, }}{ }^{1}$

${ }^{1}$ Pharmacy Student, University of Waterloo, Canada

Purpose: PopPK models designed with age or vWF as covariates on clearance are used for estimating patient PK to dose factor concentrates in patients with hemophilia. The primary objectives of this study were to identify the relationship between age and $\mathrm{vWF}$ and to determine if using patient age is as precise as patient vWF when estimating PK. Methods: Patient data from WAPPS-Hemo was used to determine the correlation between age and vWF. Various regression analyses were performed to identify the correlation equation. Three populations, each of 1000 patients, were simulated using the bootstrap method and treated with factor concentrates. A limited sampling analysis was performed, and Bayesian forecasting was used to obtain patients' PK estimates using three PopPK models. The F-test was used to compare the variability in the relative errors between the models. Results: Spearman's test showed a moderate correlation, and a quadratic equation best represented the age and vWF relationship. Relative error distributions were larger in designs with fewer samples taken (2 vs 3 collections). Designs with samples taken too close to infusion had larger relative error distributions than those spaced out. Sampling designs with $72 \mathrm{hr}$ or $96 \mathrm{hr}$ often contain undetectable, below level of quantification (BLQ), values and often reached statistical significance in the pediatric and adult populations. FVIII concentration remains detectable longer in the older adults and therefore statistical difference between models was less often reached. Conclusions: Precision of Age and vWF PopPK models are equal, and clinicians can use either of these models if proper plasma sampling times are taken to avoid obtaining below level of quantification values. If BLQ values are unavoidable due 
to sample collection schedules, patient vWF should be obtained and the vWF model should be used to estimate patient's PK. Acknowledgements: CSPS National Undergraduate Student Research Program Award recipient, University of Waterloo School of Pharmacy.

\title{
Modulation of cytochrome P450 1a1 and 1a2 by methylmercury in hepatic tissue of C57BL/6 mice
}

\author{
Mohammed Alqahtani, ${ }^{1}$ Ayman O S El-Kadi, ${ }^{2}$
}

${ }^{1}$ Ph.D. Student, Faculty of Pharmacy and Pharmaceutical Sciences, University of Alberta, Canada; ${ }^{2}$ Professor, Faculty of Pharmacy and Pharmaceutical Sciences, University of Alberta, Edmonton, Canada

Purpose: Much concern stems from the cytochrome P450 1A subfamily enzymes as they are involved in the metabolic transformation of several xenobiotics to toxic metabolites, leading to reactive intermediates formation and carcinogenesis. Mercury and polyaromatic hydrocarbons (PAHs), typified by 2,3,7,8Tetrachlorodibenzo-P-dioxin (TCDD), are persistent environmental pollutants involved in the modulation of aryl hydrocarbon (AhR) gene battery, including cytochrome P450 (CYP) genes. We previously investigated the co-exposure effect to inorganic or organic mercurials $\left(\mathrm{Hg}^{+2}\right.$ and $\left.\mathrm{MeHg}\right)$ in the presence of TCDD in vitro. Thus, the current study examined the co-exposure effect to $\mathrm{MeHg}$ or $\mathrm{Hg}^{+2}$ and TCDD on AhR-regulated genes (Cyp1a1/1a2) in vivo. Methods: Male C57BL/6 mice $(n=6)$ were injected intraperitoneally with $\mathrm{MeHg}$ or $\mathrm{Hg}^{+2}(2.5 \mathrm{mg} / \mathrm{kg})$ in the presence and absence of TCDD $(15 \mu \mathrm{g} / \mathrm{kg})$ for $6 \mathrm{~h}$ and $24 \mathrm{~h}$. Real-time PCR, Western blot, Cyp1a1/1a2 catalytic activity assays were used to determine mRNA, protein expression, and enzymatic activity, respectively. Results: MeHg significantly inhibited the TCDD-mediated induction of Cyp1a1/1a2 mRNA levels. Conversely, $\mathrm{Hg}^{+2}$ altered TCDD-mediated induction at the post-transcriptional level of Cyp1a1/1a2 protein and catalytic activity. The potential inhibitory role of heme oxygenase-1 (Ho-1) induced by MeHg suggests a modulation mechanism in the TCDD-mediated induction of Cyp1a1 as previously reported in vitro. Conclusion: The current study demonstrates for the first time that $\mathrm{MeHg}$, alongside $\mathrm{Hg}^{+2}$, can differentially modulate the TCDD-induced AhR-regulated genes (Cyp1a1/1a2) at different transcriptional levels in C57BL/6 mice liver. This work was supported by the Natural Sciences and Engineering Research Council of Canada (NSERC) Discovery Grant RGPIN 250139-107 to A.O.S.E. MA is the recipient of Saudi Government Scholarship.

\section{Investigating whether the anti-anginal drug ranolazine mitigates non-alcoholic fatty liver disease associated with type 2 diabetes}

Christina T. Saed ${ }^{1,2,3}$, Amanda A. Greenwell ${ }^{1,2,3}$, Seyed Amirhossein Tabatabaei Dakhili ${ }^{1,2,3}$, Keshav Gopal $^{1,2,3}$, Farah Eaton ${ }^{1,2,3}$, John R. Ussher ${ }^{1,2,3}$

${ }^{1}$ Faculty of Pharmacy and Pharmaceutical Sciences and ${ }^{2}$ Cardiovascular Research Centre, University of Alberta; ${ }^{2}$ Alberta Diabetes Institute, Edmonton, Alberta, Canada

Purpose: Non-alcoholic fatty liver disease (NAFLD) is defined as the presence of excess fat in the liver of individuals that are not dinking alcohol. NAFLD is a devastating disease that greatly increases a person's risk for both diabetes and cardiovascular disease. Unfortunately, there is no treatment for this devastating disease. Nearly 7 million people in Canada suffer from NALFD, and it is therefore of great importance that we better understand its pathology so that we can develop potential pharmacotherapies. Of interest, we have shown that ranolazine, a second-line agent for angina, can also mitigate NAFLD in obesity. Our aim was to determine whether ranolazine has these same actions in NAFLD associated with type 2 diabetes (T2D). Methods: T2D was induced in 8-week-old male C57BL/6J mice (Jackson Laboratories) by 12-weeks of high-fat diet supplementation with a single low-dose injection of streptozotocin [75 mg/kg] at 4-weeks. Lean control mice were fed a low-fat diet for 12 weeks. At 8-weeks, lean and T2D mice treated with either 
vehicle or ranolazine $(50 \mathrm{mg} / \mathrm{kg}$ ) once daily via oral gavage for 30-days. following which we assessed hepatic steatosis and glucose homeostasis. Results: Contrasting our previous findings in obesity-related NAFLD, ranolazine treatment did not improve glycemia or hepatic steatosis in T2D-related NAFLD, as indicated by similar glucose tolerance and hepatic triacylglycerol content as their vehicle treated T2D counterparts. Conclusion: Our results suggest that ranolazine's potential utility as a therapy for NAFLD may be limited to obese individuals but not those with T2D, and it will be important for future research to identify the reasons explaining these discrepancies.

\section{Fluconazole Exerts a Protective Role against Pressure Overload-Induced Cardiac Hypertrophy in Rats}

$\underline{\text { Sherif M. Shoieb, }}{ }^{1}$ Jody Levasseur, ${ }^{2}$ Heidi Silver, ${ }^{3}$ Jason R.B. Dyck, ${ }^{4}$ Ayman O.S. El-Kadi, ${ }^{5}$

${ }^{1} \mathrm{PhD}$ candidate, Faculty of Pharmacy and Pharmaceutical Sciences, University of Alberta, Edmonton, Alberta, Canada, Canada; ${ }^{2}$ Animal Physiology Technician, Cardiovascular Research Centre, Department of Pediatrics, Faculty of Medicine and Dentistry, University of Alberta, Edmonton, Alberta, Canada, Canada; ${ }^{3}$ Laboratory Technician, Cardiovascular Research Centre, Department of Pediatrics, Faculty of Medicine and Dentistry, University of Alberta, Edmonton, Alberta, Canada, Canada; ${ }^{4}$ Professor, Cardiovascular Research Centre, Department of Pediatrics, Faculty of Medicine and Dentistry, University of Alberta, Edmonton, Alberta, Canada, Canada; ${ }^{5}$ Professor, Faculty of Pharmacy and Pharmaceutical Sciences, University of Alberta, Edmonton, Canada

Purpose: Cytochrome P450 1B1 (CYP1B1) is known to be involved in the pathogenesis of several cardiovascular diseases such as cardiac hypertrophy, through the formation of cardiotoxic metabolites named as midchain hydroxyeicosatetraenoic acids (HETEs). Recently, we have demonstrated that fluconazole, an antifungal agent, decreases the level of mid-chain HETEs in human liver microsomes, inhibits human recombinant CYP1B1 activity and protects against angiotensin II-induced cellular hypertrophy in H9c2 cells. Therefore, the overall objectives of the present study were to elucidate the potential cardioprotective effect of fluconazole against cardiac hypertrophy induced by abdominal aortic constriction (AAC). Methods: Male Sprague-Dawley rats were randomly assigned into four groups; sham control rats, fluconazole-treated $(20 \mathrm{mg} / \mathrm{kg}$ daily for 4 weeks, intraperitoneal) sham rats, AAC rats and fluconazole-treated $(20 \mathrm{mg} / \mathrm{kg}) \mathrm{AAC}$ rats. Baseline and 5 weeks post-AAC echocardiography were performed. Gene and protein expression were measured using real-time PCR and WB analysis, respectively. The level of mid-chain HETEs was determined using liquid chromatography-mass spectrometry (LC/MS). Results: Echocardiography results showed that fluconazole significantly reversed the AAC-induced left ventricular hypertrophy, as it ameliorated the AAC-mediated increase in left ventricular mass and several wall measurements. Also, fluconazole significantly prevented the AACmediated increase of hypertrophic markers. The anti-hypertrophic effect of fluconazole was associated with a significant inhibition of CYP1B1 at the gene and protein levels and a reduction in the formation rate of midchain HETEs. Conclusion: The current study demonstrates that fluconazole protects against left ventricular hypertrophy. The findings of the present work highlight the potential repurposing of fluconazole as a CYP1B1 inhibitor for the protection against cardiac hypertrophy and a possible treatment for heart failure. Support: This work was supported by a grant from the Canadian Institutes of Health Research [PS168846] to A.O.S.E. S.M.S. is the recipient of Alberta Innovates Graduate Student Scholarship and Alberta Graduate Excellence Scholarship. 


\section{Modulation of aryl hydrocarbon receptor (AhR)-regulated genes expression by arsenic trioxide (ATO) in Hepa1c1c7 cells}

Mahmoud El-Ghiaty, ${ }^{1}$ Mohammed Alqahtani, ${ }^{1}$ Ayman O S El-Kadi, ${ }^{2}$

${ }^{1} \mathrm{Ph} . \mathrm{D}$. Student, Faculty of Pharmacy and Pharmaceutical Sciences, University of Alberta, Canada

${ }^{2}$ Professor, Faculty of Pharmacy and Pharmaceutical Sciences, University of Alberta, Edmonton, Canada

Purpose: Arsenic is a ubiquitous occupational and environmental contaminant that imposes threat to humans through its toxicity and carcinogenicity. However, some arsenicals are exploited for their remedial effects. Arsenic trioxide (ATO) is successfully applied in the treatment of acute promyelocytic leukemia (APL) and is currently under investigations for treating other types of cancer. Both inorganic arsenic and its methylated metabolites have been shown to modulate aryl hydrocarbon receptor (AhR)-regulated phase I and phase II xenobiotic metabolizing enzymes which are involved in the carcinogenic and cytoprotective pathways, respectively. In this study we examined the impact of ATO on AhR phase I and phase II enzymes typified by cytochrome P450 1a1 (Cyp1a1) and NADPH:quinone oxidoreductase (Nqo1), respectively. Methods: Murine hepatoma (Hepa1c1c7) cells were treated with ATO $(0.63,1.25$ and $2.5 \mu \mathrm{M})$ in the absence or presence of $1 \mathrm{nM}$ of the archetypal AhR ligand, 2,3,7,8-tetrachlorodibenzo-p-dioxin (TCDD). Cypla1 and Nqo1 expression was determined at both mRNA and protein levels using qPCR and Western blot analysis, respectively. MTT Assay was used to assess cell viability. Results: Cell viability was not affected by all concentrations of ATO used. ATO caused a concentration-dependent increase in TCDDmediated induction of Cyp1a1 at mRNA and protein levels. However, Cyp1a1 mRNA, but not protein, was induced by ATO alone. On the other hand, ATO, alone or in the presence of TCDD, significantly increased Nqo1 at both mRNA and protein levels in a concentration-dependent manner. Conclusion: Our study demonstrated that ATO induces the AhR-regulated Cyp1a1 and Nqo1 enzymes with subsequent implications on cellular response to xenobiotics co-exposure. However, further studies are required to explain the mechanism of this behavior. This work was supported by Natural Sciences and Engineering Research Council of Canada (NSERC) Discovery Grant RGPIN 250139 to A.O.S.E. M.A.E. is the recipient of Pharmacy PhD Alumni Graduate Student Scholarship.

\section{Hepatic glutathione S-transferase activity shows sex, strain and species differences between rodents and human: Implications for safety pharmacology/toxicology and first-in-human trials}

Michael Doerksen, ${ }^{1}$ Denny Seo, ${ }^{2}$ Alexander Smith, ${ }^{3}$ Abby Collier, ${ }^{4}$

${ }^{1} \mathrm{PhD}$ Student, ${ }^{2} \mathrm{PharmD}$ Candidate, Research Associate, ${ }^{4}$ Professor, Faculty of Pharmaceutical Sciences, The University of British Columbia, Canada

Purpose: Glutathione S-transferases (GSTs) are phase II enzymes involved in antioxidant defence, drug metabolism, and cell signalling. Here, we investigated differences in glutathione conjugation between rodent (mice and rat) strains, sexes, and with a direct comparison to humans. Methods: Subcellular liver fractions (cytosol and microsomes) were incubated with selective GST substrates to evaluate specific activity. General GST activity was probed using 1-chloro-2,4-dinitrobenzene in both cytosolic and microsomal fractions. Isoform-selective substrates, ethacrynic acid, 3,4-dichloronitrobenzene, and 4-nitro2-benzyl chloride, were used to investigate the cytosolic subfamilies GST-P, GST-M, and GST-T, respectively. Where possible, sex comparisons within a strain were analyzed. Results: There were significant differences between rodent and human hepatic GST activities. Cytosolic "total" GST activities were significantly greater than human for all mice strains, and for male Sprague Dawley and Wistar Han rats $(\mathrm{p}<0.001)$. Sex-differences in cytosolic GST activities were observed for all rodents, except Wistar rats $(\mathrm{p}<0.05)$. Microsomal GST, GST-M, and GST-T activities were significantly greater than the human 
for all rodent strains $(\mathrm{p}<0.05)$. Significant sex differences in rat GST-M and GST-T activities were observed, but only for GST-M in mice ( $\mathrm{p}<0.0001$ ). Lastly, there were no significant differences between rat and human GST-P activities although some mice strains (BALB/c, C57BL/6, male CD-1, male B6C3F1) showed significantly greater activities. Conclusion: These data suggest significant differences in glutathione conjugation between humans and rodents, including differences between strains, and sex differences within strains. We highlight the importance of careful model selection for pre-clinical animal models where GST is the primary metabolic pathway. Extrapolating to first-in-human trials, and/or safety pharmacology/toxicology studies, should be carefully considered for GST-specific drug metabolism and pharmacokinetics.

\section{6-(R/S)-Hydroxyeicosatetraenoic acids (HETEs) induce human CYP1B1 through transcriptional and allosteric mechanisms}

$\underline{\text { Rahmat Hidayat, }},{ }^{1}$ Sherif M Shoieb, ${ }^{2}$ Mahmoud El-Ghiaty, ${ }^{3}$ Mohammed Alqahtani, ${ }^{3}$ Ayman OS El-Kadi,

${ }^{1}$ Master Student, ${ }^{2}$ Graduate Student, ${ }^{3} \mathrm{Ph}$.D. Student, ${ }^{4}$ Professor, Faculty of Pharmacy and Pharmaceutical Sciences, University of Alberta, Edmonton, Canada

Purpose: Several reports from our laboratory and others demonstrated the direct contribution of cytochrome P450 1B1 (CYP1B1) enzyme and its associated cardiotoxic mid-chain hydroxyeicosatetraenoic acid (HETEs) metabolites in the development of cardiac hypertrophy and heart failure. In addition, we have previously reported that the subterminal HETE, 16-HETE, confer cardioprotection against cardiovascular diseases. Therefore, we investigated the effect of 16-(R/S)-HETE on CYP1B1 at mRNA, protein as well as catalytic activity levels. Methods: We incubated human fetal ventricular cardiomyocytes (RL-14) cell lines, recombinant human CYP1B1, and human liver microsomes in the presence and the absence of 16-(R/S)HETE. Thereafter, real-time PCR, Western blot analysis, and CYP1B1-dependant EROD assay were performed to determine the level of CYP1B1 mRNA, protein, and catalytic activity levels, respectively. To examine whether a similar effect will happen with other CYP that is regulated by the same transcription factors, aryl hydrocarbon receptor, we tested the effect of 16-(R/S)-HETE on CYP1A2 catalytic activity in human recombinant CYP1A2 using MROD assay. Results: Our results showed that 16-HETE significantly upregulated CYP1B1 at mRNA and protein levels in RL-14 cell lines. Surprisingly, 16-(R/S)-HETE significantly increased CYP1B1 activity in RL-14 cells, recombinant human CYP1B1, and human liver microsomes. On the contrary, 16-(R/S)-HETE significantly inhibited CYP1A2 catalytic activity mediated by the recombinant human CYP1A2 and human liver microsomes. Conclusion: Our study provides the first evidence that 16-(R/S)-HETE increases CYP1B1 gene expression, protein at least in part, through the transcriptional mechanism, and CYP1B1 at catalytic activity in recombinant and liver microsome possibly through an allosteric mechanism.

\section{A role for the steroid metabolite recycling enzyme Beta-glucuronidase in prostate cancer progression}

\section{Brandon Haefling, ${ }^{1}$ Blair Macdonald, ${ }^{2}$ Manrose Mann, ${ }^{3}$ Alexander Smith, ${ }^{4}$ Karla Williams,,${ }^{5}$ Michael} Cox, ${ }^{6}$ Michael Coughtrie, ${ }^{7}$ Abby Collier, ${ }^{8}$

${ }^{1}$ MSc Student - Pharmaceutical Sciences, The University of British Columbia, Canada; ${ }^{2}$ PharmD Student, The University of British Columbia, Canada; ${ }^{3}$ Pharmacist, The University of British Columbia, Canada ${ }^{4}$ Research Associate, Faculty of Pharmaceutical Sciences, The University of British Columbia, Vancouver, Canada; ${ }^{5}$ Assistant Professor, Faculty of Pharmaceutical Sciences, The University of British Columbia, vancouver, Canada; ${ }^{6}$ Associate Professor and Senior Scientist, The University of British Columbia and Vancouver Prostate Centre, Canada; ${ }^{7}$ Professor and Dean, Faculty of Pharmaceutical Sciences, The 
University of British Columbia, Canada; ${ }^{8}$ Professor; Director of Bachelor of Pharmaceutical Sciences degree program, Faculty of Pharmaceutical Sciences, The University of British Columbia, Canada

Purpose: Testosterone (T) and dihydrotestosterone (DHT) are primary drivers of prostate cancer growth on the molecular and physiological level. While most investigations study androgen receptor interactions, or androgen production, steroid clearance and deconjugation are overlooked. $\beta$-glucuronidase $(\beta \mathrm{G}, \mathrm{EC}$ 3.2.1.31) deconjugates inactive $\mathrm{T}$ and DHT glucuronides, recycling active $\mathrm{T}$ and DHT that may bind the androgen receptor. This study investigated the role of $\beta \mathrm{G}$ deconjugation in prostate cancer (PCa) using cell lines and prostate tissue samples representative of normal, benign, and malignant PCa. We hypothesized that $\beta \mathrm{G}$ expression and activity would increase with PCa progression. Methods: Prostate cells were obtained from American Type Culture Collection (ATCC) or gifted by Dr. Michael Cox, and bulk cultured. Prostate tissue lysates were purchased from Origene and Novus Biologicals. Cell lysates were prepared by homogenization and protein quantified by BCA assay. Protein expression was assessed by western blotting and enzyme activities using 4-methylumbelliferone (4MU) and 4-methylumbelliferyl- $\beta$-D-glucuronide (4MUG) as substrates. Results: Genomic copy number and mRNA expression of $\beta G$ increased significantly with prostate cancer progression and drug resistance in two public databases we analyzed. Functionally, LNCaP, C4-2, and PC3 cells showed greatest $\beta$ G activity at $98 \pm 4,144 \pm 30$, and $287 \pm 68 \mathrm{pmol} / \mathrm{min} / \mathrm{mg}$ respectively. In normal, hyperplasia, adenoma and adenocarcinoma prostate tissues, $\beta \mathrm{G}$ activity was greatest in adenoma and adenocarcinoma samples at $750 \pm 72$ and $2,588 \pm 78 \mathrm{pmol} / \mathrm{min} / \mathrm{mg}$ respectively. Cell lines and tissues showed conjugation rates that were up to 3 -fold higher but $\beta G$ increases were up to 12 -fold with progression to adenocarcinoma. Conclusion: $\beta G$ activity increases with prostate cancer progression in cell lines and prostate tissues suggesting steroid deconjugation may play a role in PCa. PC3, C4-2 and LNCaP cell lines may serve as appropriate in vitro models investigating deconjugation pathways and cancer growth.

\section{Effect of Wild blueberries in the MPTP mouse model of Parkinson's Disease}

\section{$\underline{\text { Sunisha Aryal, }},{ }^{1}$ Taylor Skinner, ${ }^{2}$ Bronwyn Bridges, ${ }^{2}$ John Weber, ${ }^{3}$ Jenika Marshall, ${ }^{4}$ Nicole Head, ${ }^{5}$} Sasha Power, ${ }^{6}$

${ }^{1}$ Graduate Student, ${ }^{2}$ Graduate Student, ${ }^{3}$ Professor, ${ }^{4}$ Research Assistant, ${ }^{5}$ Student, ${ }^{6}$ Undergraduate Student, Memorial University of Newfoundland, Canada

Purpose: Neurodegenerative disorders such as Parkinson's disease (PD) cause debilitating motor dysfunction and cognitive symptoms. Natural products such as blueberry extracts contain compounds that may reduce inflammation and neuronal degeneration. We have found that blueberry extracts protect cultured neurons and microglia from glutamate and alpha-synuclein exposure [1]. In this study, extracts from wild blueberries were tested for potential neuroprotective effects in mice using the 1-methyl-4-phenyl1,2,3,6-tetrahydropyridine (MPTP) model of PD. Methods: 36 C57BL/6 strain male mice (Charles River) weighing approx. $22 \mathrm{~g}$ were housed individually with free access to regular chow (9.00 to $15.00 \mathrm{hrs})$ and then with $7 \mathrm{~g}$ of mash (15.00 to $9.00 \mathrm{hrs)}$ consisting of rodent chow and water (1:1) or ground blueberry fruits (5\% blueberry fruit, rodent chow, and water). Mice underwent 5 days of injections $(15 \mathrm{mg} / \mathrm{kg})$ with sterile water (12 on a normal diet), or with MPTP dosed by weight (12 normal diet; 12 blueberry diet). After the regimen, mice were tested on rotarod, dark light box, and open field. Following the tests, mice were euthanized, and brains were prepared for western blots. Results: There were no significant differences between saline injected normal diet mice, MPTP injected normal diet mice, and MPTP injected blueberry enriched diet mice in the behavioral tests at $\mathrm{p}<0.05$ (one-way anova). Currently, we are completing western blot analysis of alpha synuclein and tyrosine hydroxylase in brain tissue. Conclusion: Although not significant, the behavioural data of our study is similar to others in the literature [2]. Analysis of brain tissue is yet to be completed and may show significant results at a cellular level. Overall, additional 
experiments and analysis are needed to determine a potential protective effect of blueberry enriched diets in PD.

\title{
Pharmacodynamic Analysis of Trabecular Bone in Ovariectomized Rats Treated with Bone- Targeting Parathyroid Hormone Analogues
}

\author{
Conor O'Croinin, ${ }^{1}$ Benjamin O'Croinin, ${ }^{1}$ Ali Aghazadeh Habashi, ${ }^{2}$ Neal Davies, ${ }^{3}$ Michael Doschak, ${ }^{4}$ \\ ${ }^{1}$ Summer Student Undergraduate Trainee, University of Alberta, Canada; ${ }^{2}$ Assistant Professor, Idaho State \\ University, Canada; ${ }^{3}$ Dean and Professor, University of Alberta, Canada; ${ }^{4}$ Professor, Faculty of Pharmacy \\ \& Pharmaceutical Sciences, University of Alberta, Edmonton, Canada
}

Purpose: Osteoporosis is characterized by increased bone fragility and risk of fracture. Parathyroid hormone (PTH) drugs are an effective anabolic therapy for strengthening bone micro-architecture, however systemic exposure of the peptide hormone drug at supra-physiological concentration leads to potential side effects in off-target tissues that also express PTH receptors. Our study objective was to target drug action preferentially to bone tissue, using bisphosphonate-PTH (BP-PTH) drug conjugates. An incremental increase in trabecular bone structure is expected by in vivo micro-Computed Tomography (micro-CT), using a dose-response experimental design. Methods: In vivo micro-CT scan data was obtained for 49 rat tibia, representing measurements conducted in ovariectomized rats at monthly intervals over 17 wks. Daily drug treatments commenced in all rats from the $8 \mathrm{wk}$ time-point until $17 \mathrm{wk}$ study endpoint. Rats were dosed either PTH, BP-PTH conjugate at one of three doubling concentrations (Conj-10, Conj-20, and Conj40), or saline vehicle negative control. Micro-CT images were reconstructed using back-projection with a modified Feldkamp algorithm. Each dataset was reoriented using vendor bundled software, trabecular bone micro-architectural data extracted and measured for given region of interest (ROI) selections through 200 slices, reconstructed at orthotropic $18 \mu \mathrm{m}$ resolution, to determine trabecular morphometric indices such as percent bone volume (\%BV), trabecular thickness and separation, and 3-Dimensional connectivity. Results: Statistical analysis of variance (ANOVA) indicated that week $17 \%$ BV was significantly different between Vehicle, Conj-40, and systemic PTH. Trabecular bone morphometric indices were not different between Conj-10 and Conj-20 dosed groups, however, at week 17 the trabecular thickness of Conj-20 and Conj-40 significantly exceeded that of Conj-10 and PTH. Conclusion: Administration of both PTH and BP-PTH was measured to elicit an anabolic bone response. A clear anabolic dose-response relationship was measured for BP-PTH, notably for trabecular thickness, where BPPTH outperformed systemic PTH drug exposure in ovariectomized rats. 


\title{
Supplement
}

\section{GSK/CSPS undergraduate students research award presentations scheduled to be presented at the cancelled 2020 annual CSPS meeting}

\author{
Factors associated with patient success in a self-medication program in a rehabilitation setting in \\ Halifax, Nova Scotia.
}

Chandni Sehgal, ${ }^{1,2}$ Kathleen MacMillan,,${ }^{2,3}$ Carl Kooka, ${ }^{2}$ Heather Neville, ${ }^{2}$ Michele Haire, ${ }^{2}$ Claudia Harding, ${ }^{2}$ Monique MacFarlane-Conrad, ${ }^{2,3}$ Steve MacKay, ${ }^{2}$ Anita Mountain, ${ }^{2,3}$ Lisa Nodwell, ${ }^{2}$ Christine Short, ${ }^{2,3}$ Kelly White Jankari ${ }^{2}$

${ }^{1}$ College of Pharmacy, Dalhousie University; ${ }^{2}$ Nova Scotia Health Authority; ${ }^{3}$ Department of Medicine, Dalhousie University

Purpose: The Nova Scotia Rehabilitation and Arthritis Centre has had a long-standing self-medication program (SMP), where patients learn to manage their own medications. The Drugs Regimen Unassisted Grading Scale (DRUGS) is a validated tool that can be used to evaluate SMP progress. Studies to date have not determined factors that are associated with good DRUGS scores. We studied the factors related to medication management and adherence in a self-medication program. Methods: Patients enrolled in an SMP at the Nova Scotia Rehabilitation and Arthritis Centre were invited to participate. Participants' capacity to manage their medications was evaluated prior to SMP and post-SMP using the DRUGS score and by calculating medication error rates. Follow-up data obtained at four weeks and six months were used to calculate the proportion of days covered (PDC) to assess adherence. ANOVA and chi-square analyses were performed. Results: Ninety patients were eligible for the study. Patient average age was 56.9 years and average length of stay was 65.1 days. The mean DRUGS score increased from $86.3 \%( \pm 16.9 \mathrm{SD})$ preSMP to $92.3 \%$ ( \pm 13.9 SD) post-SMP. Mean medication errors were 13.8 ( $\pm 32.6 \mathrm{SD}$ ) per patient, or 0.04 $( \pm 0.1 \mathrm{SD})$ per dose. Patients who made $>30$ errors were significantly associated with final DRUGS scores of $<75 \%(\mathrm{p}=0.00016)$ and less likely to have final DRUGS scores of $100 \%(\mathrm{p}=0.0027)$. Likewise, error rates of $>10 \%$ were significantly associated with final DRUGS scores of $<75 \%(\mathrm{p}=0.00016)$. No significant associations were found between PDC (mean score $=1.0 \pm 0.2 \mathrm{SD}$ ) and other factors, including DRUGS score. Conclusions: Patients enrolled in an SMP who had good medication management capacity also made fewer medication errors. Medication adherence after discharge was very high and may not be related to medication management abilities in hospital. Acknowledgements: This project was funded by the Nova Scotia Health Authority Research Fund and the Nova Scotia Rehabilitation Endowment Fund. The authors have no financial or personal relationships with commercial entities to disclose.

\section{Platelets selectively regulate the release of BDNF, but not that of proBDNF}

Jessica Le Blanc $^{1,2}$, Samuel Fleury ${ }^{1,2}$, Imane Boukhatem ${ }^{1,2}$, Jean-Christophe Bélanger ${ }^{1,2}$, Mélanie Welman ${ }^{1}$, Marie Lordkipanidzé1,2

${ }^{1}$ Research Center, Montreal Heart Institute; ${ }^{2}$ Faculty of Pharmacy, Université de Montréal

Background: The Brain-Derived Neurotrophic Factor (BDNF) is a member of the neurotrophin family, known to play a role in long-term memory and cognition. Its precursor, proBDNF, has the opposite biological actions and induces neuronal death. Although initially discovered in the brain, BDNF is abundantly present in platelets and can be released into circulation upon their activation. It is not known whether platelets also 
contain proBDNF, and whether they regulate its bioavailability in blood. Purpose: To determine whether platelets contain a releasable pool of proBDNF. Methods: Platelet-rich plasma was isolated from healthy volunteers. The presence of BDNF and proBDNF was assessed by Western blotting and flow cytometry. Platelets were activated with various agonists and levels of BDNF and proBDNF in platelets and their supernatant were measured by ELISA. Results: Western blotting on platelet lysates showed a clear band at the expected molecular weight for proBDNF, alongside human cortex lysates used as positive controls. By flow cytometry, proBDNF was localized in the intracellular compartment and fractionation experiments confirmed it was in the cytosol. The molar ratio of proBDNF to BDNF was 1:5 in platelets, but 10:1 in plasma. Upon activation, platelets released most of their BDNF into plasma, but no increase in proBDNF plasma levels was seen. Conclusion: To our knowledge, we are the first to establish the presence of proBDNF in human platelets. Recent advances have shown an imbalance in the proBDNF/BDNF ratio in the brains of patients with neuropsychiatric diseases. The platelet proBDNF/BDNF ratio could represent a non-invasive biomarker of neurocognitive health.

\section{The Effect of Gold Nanoparticle Protein corona formation on its imaging capability of tumour tissue}

Tushar Upreti ${ }^{1}$, Nasrin Hooshmand ${ }^{2}$, Mostafa A. El-Sayed ${ }^{2}$, Hagar I. Labouta ${ }^{1}$

${ }^{1}$ College of Pharmacy, University of Manitoba, Winnipeg, Manitoba R3E 0T5, Canada; ${ }^{2}$ Laser Dynamics Laboratory, School of Chemistry and Biochemistry, Georgia Institute of Technology, Atlanta, GA 30332, United States

Gold Nanoparticles (AuNP) have high potential as a diagnostic tool of tumour tissue based on their surface plasmon resonance. Fluorophores situated in the vicinity of AuNP usually experience quenching of their fluorescence. Question remains as to how much, if any, AuNP can quench the autofluorescence of the tumour extracellular matrix (ECM). Little is also known on how the interaction of injected AuNP with the different plasma proteins, such as albumin, may alter their interaction with the ECM and their bioimaging capability. We hypothesize that the interaction of albumin with AuNP would result in a protein corona formation on the surface of AuNP; thus, causing a decrease in the quenching property of AuNP when interacting with collagen (key component of ECM). To test this hypothesis, we synthesized AuNP $(15.7 \pm 0.5 \mathrm{~nm})$ and investigated their interaction with bovine serum albumin, collagen and a combination thereof at conditions simulating in vivo conditions at different time intervals using UV-Vis and fluorescence spectroscopy. Our UV-Vis results show that albumin deposition on AuNP resulted in less interaction with the collagen (evident by a smaller red shift of the plasmon peak, $5 \mathrm{~nm}$ versus $10 \mathrm{~nm}$ for naked particles) and higher stability evident by lack of plasmon peak broadening observed for naked AuNP. Quenching of collagen autofluorescence by AuNP was reduced on surface deposition of albumin on AuNP. This suggests that AuNP interaction with tumour ECM is governed by protein corona formation. Design of nanodiagnostics with a high potential of clinical translation should therefore account for the variation in characteristics of AuNP in the presence of plasma proteins.

\section{Pharmacokinetics of Native and Nano-Formulated Innate Defense Regulator Peptides}

$\underline{\text { Colin Blackadar }}^{1, *, \#}$, Tullio V. F. Esposito ${ }^{1,2, *}$, Daniel Pletzer ${ }^{3,4}$, Cristina Rodríguez-Rodríguez ${ }^{1,5,6}$, Evan

Haney $^{3}$, Robert E. W. Hancock ${ }^{3}$, Katayoun Saatchi ${ }^{1}$, Urs O. Häfeli ${ }^{1,2, \neq}$

${ }^{1}$ Faculty of Pharmaceutical Sciences, University of British Columbia, Vancouver, Canada; ${ }^{2}$ Department of Pharmacy, Faculty of Health and Medical Sciences, University of Copenhagen, Copenhagen,

Denmark; ${ }^{3}$ Centre for Microbial Disease and Immunity Research, Department of Microbiology and Immunology, Faculty of Science, University of British Columbia, Vancouver, Canada; ${ }^{4}$ Department of Microbiology and Immunology, University of Otago, Dunedin, New Zealand; ${ }^{5}$ Department of Physics and Astronomy, Faculty of Science, University of British Columbia, Vancouver, Canada; ${ }^{6}$ Centre for Comparative Medicine, University of British Columbia, Vancouver, Canada 
Background: Innate defense regulators (IDRs) are short, cationic peptides with antimicrobial and immunomodulatory activity. Many factors are limiting their clinical translation, namely solubility issues, toxicity, and a poor understanding of their in vivo behavior/biodistribution. Purpose: To develop a polymerbased formulation of IDRs that reduces aggregation and toxicity, and to investigate the pharmacokinetics of the native and formulated IDRs. Methods: IDRs (IDR1002 and IDR1018) were conjugated to a nanoscale dendrimer via a disulfide linkage. The formulations were characterized using DLS and ${ }^{1} \mathrm{H}-\mathrm{NMR}$ and their toxicity and immunomodulatory profile were evaluated against various cell types. Using ${ }^{67}$ Ga-labeled IDRs, the pharmacokinetics of the native and formulated peptides was assessed using SPECT/CT via various routes (IV, SC, IP, IT) and dose levels (2.5-40 mg/kg). Results: At low IV doses $(2.5 \mathrm{mg} / \mathrm{kg}$ ) the native IDRs were rapidly cleared by the kidneys (IDR1002) and liver (IDR1018). At higher IV doses (>5 mg/kg), the peptides precipitated causing lethal embolization of the lung capillaries. For other administration routes, the IDRs were rapidly absorbed at lower doses and formed local precipitation-based depots as the dose escalated. The formulation increased the plasma half-life of the IDRs upon IV injection from 1 minute to over 3 hours. The formulation also decreased the cytotoxicity and hemolytic activity of the peptides while maintaining their immunomodulatory functions.

Conclusion: IDRs are important new compounds to treat infectious and inflammatory diseases. However, toxicity and pharmacokinetic hurdles exist. Our formulation addresses a number of these issues.

(*Contributed equally.)

\title{
An Investigation into CRISPR Cas12a's in-vitro ssDNA Interactions.
}

$\underline{\text { Winston Chan }}^{1}$, Margot Karlikow ${ }^{1}$, Keith Pardee ${ }^{1}$.

\section{${ }^{1}$ University of Toronto, Leslie Dan Faculty of Pharmacy}

Background; While Cas12a's properties and requirements for cleaving dsDNA are well characterised, with a PAM, crRNA, and direct repeat sequence required for RNA targeted cis-cleavage and subsequent trans-cleavage activation, little is known with regards to Cas12a's cleavage properties and interactions with ssDNA. Purpose; The purpose of this study was to determine the interactions of FnCas12'a with ssDNA, to aid the development of a novel in vitro technology. Methods: We used urea denaturing polyacrylamide RNA gels to determine the activity of FnCas12a on ssDNA. Results: In this study, we demonstrated that in the presence of a ssDNA fragment (consisting of a spacer sequence adjacent to a direct repeat) and a T7 RNA Polymerase, FnCas12a's cleavage of the ssDNA fragment produces four distinct DNA bands of $21 \mathrm{bp}, 37 \mathrm{bp}, 47 \mathrm{bp}, 88 \mathrm{bp}$, indicating the presence of 2 distinct cleavage sites. Conclusion: Additional research will need to be conducted to fully elucidate the properties of interaction with ssDNA. This project is the recipient of the 2020 GSK student award.

\section{Characterization of a poly(Acrylic acid)-grafted-Cellulose NanoCrystal Bio-adhesive Nano-gel for Local Antineoplastic Drug Delivery in Colorectal Cancer Treatment:}

\author{
$\underline{\text { Julian Ethier-Hopwood }^{1} \text {, Waleed Mohammed-Saeid }}{ }^{1,2}$, Mohammad Reza Vakili ${ }^{1}$, Behzad Ahvazi ${ }^{3}$, Afsaneh \\ Lavasanifar $^{1}$ \\ ${ }^{1}$ Faculty of Pharmacy and Pharmaceutical Sciences, University of Alberta, Edmonton, AB, Canada; \\ ${ }^{2}$ College of Pharmacy, Taibah University, Medina, Saudi Arabia: ${ }^{3}$ InnoTech Alberta, Edmonton, AB, \\ Canada
}

Background: Colorectal Cancer (CC) contributes to $12 \%$ of Canada's total cancer diagnoses per year. Secondary to surgery, systemically administrated antineoplastic drugs, e.g. cisplatin (CDDP) are used to treat $\mathrm{CC}$ and are not well tolerated due to adverse effects. Efficient local delivery of antineoplastic to the 
tumor site can reduce systemic toxicity and enhance local effect. Purpose: We propose the development of a bio-adhesive nanogel composed of polyacrylic acid (PAA)-grafted-cellulose nanocrystal (CNC) for local delivery of CDDP in CC, that will decrease the systemic toxicity and prolong the release of the drug upon local administration. Methods: CNC-PAA-CDDP formulations were characterized for their $\zeta$-potential and size using dynamic light scattering (DLS). ICP-MS technique and dialysis method were used to evaluate the in vitro release of CNC-PAA-CDDP formulations. In vitro cytotoxicity of CDDP in developed formulations was evaluated in human colorectal carcinoma cell line, HCT 116, using MTT assay. Results: DLS measurements showed nano-sized CNC-PAA-CDDP complexes with an average diameter of $220 \pm 2 \mathrm{~nm}$. The release study, CDDP alone showed $80 \%$ release compared to $35-50 \%$ drug release for CNC-PAACDDP formulations. The degree of PAA grafting affected the release of CDDP from complexes. CNCPAA-CDDP showed 4-fold higher $\mathrm{IC}_{50}$ values compared to free drug, reflecting a slow drug release from this formulation as shown by MTT. Conclusion: The characterization of the CNC-PAA-CDDP nanogel is the first step in achieving a new local delivery agent for $\mathrm{CC}$. This therapy may have better patient tolerability by potentially minimizing adverse effects from systemic drug exposure.

\section{Optimization of Peptide-Modified Gemini Surfactant-Based Gene Delivery System for Dental Application}

$\underline{B r i t t a n y}$ Scutchings $^{1}$, Fatemeh Mohabatpour ${ }^{2,3}$, Petros Papagerakis ${ }^{2,3}$ and Ildiko Badea ${ }^{1}$ ${ }^{1}$ College of Pharmacy and Nutrition, ${ }^{2}$ College of Dentistry, ${ }^{3}$ Division of Biomedical Engineering, University of Saskatchewan, Saskatoon, Canada

Background: Safe and efficient vectors are fundamental for gene transfer and non-viral systems have advantage over viral vectors as they do not elicit an immune response. Cationic gemini surfactants can condense DNA efficiently and show promising characteristics for in vitro and in vivo applications. Due to a unique structure GS can be modified with peptides to enhance transfection efficiency and improve cellbinding. Purpose: To optimize non-viral gene delivery systems based on cationic lipids, GS and DOTAP, to improve expression of key signaling factors in dental stem cells. Methods: Plasmid DNA encoding a key transcriptional factor and green fluorescent protein (GFP) were complexed with GS or DOTAP in the presence of helper lipid, DOPE. Physical characteristics (size and surface charges) of GS and DOTAP lipoplexes were determined via dynamic light scattering, laser Doppler micro-electrophoresis Zetasizer, and transmission electron microscopy. Dental stem cells were transfected with lipoplexes and GFP expression was monitored at 3, 5 and 24 hours by fluorescent microscopy. Results: At nitrogen:phosphate ratio 5 the size of GS lipoplexes was $115.9 \mathrm{~nm}$ and DOTAP lipoplexes were $95.3 \mathrm{~nm}$. Polydispersity index was less than.25 for both formulations. Zeta potential was $23.8 \mathrm{mV}$ and $26.7 \mathrm{mV}$, respectively. Transfection showed highest GFP expression at 5 hours with GS lipoplexes illustrating higher efficiency compared to DOTAP lipoplexes. Conclusion: GS and DOTAP lipoplexes have potential to be used as gene delivery system for dental tissue regeneration due to their transfection efficiency. Future work involving gene and protein expression via qRT-PCR and western-blot will add clinical relevance.

\section{Effects of wild Newfoundland blueberries in models of Parkinson's disease}

\section{Taylor Skinner, Sunisha Aryal, Sasha R. Power, Nicole Head and John T. Weber}

School of Pharmacy, Memorial University, St. John’s, Canada

Background: Parkinson's disease (PD) is characterized by progressive loss of neurons in the substantia nigra (SN). Glutamate and alpha-synuclein can activate microglia, which release reactive oxygen species, ultimately leading to neuronal death. Blueberries are high in polyphenols, which are potent antioxidants that 
could reduce neuronal degeneration. Purpose: Blueberry extracts were tested for protective effects in cultured SN cells, and with the MPTP mouse model of PD. Methods: SN neurons were treated with 100 $\mathrm{uM}$ glutamate or $100 \mathrm{ng} / \mathrm{ml}$ of alpha-synuclein for 24 hours with or without blueberry extract. Cells were stained with DAPI, which labels cell nuclei. C57BL/6 mice were fed a mash of chow and water (24 mice) or of ground blueberry fruits. Mice underwent 5 days of injections with saline (12 on normal diet), or with MPTP (12 normal diet/12 blueberry diet), and were tested on Open Field, Dark Light Box and Rotarod tests after the injection regimen. Results: Glutamate and alpha-synuclein caused a loss of SN cells at 24 hours, indicated by DAPI staining. Blueberry extracts provided no protection from cell death. There were no significant differences in the behavioural tests between saline injected mice, MPTP mice on a normal diet, and MPTP mice on a blueberry-enriched diet. However, all results trended towards significance, suggesting expected deficits were present and then partially recovered by the blueberry diet. Conclusion: Surprisingly, blueberry extracts did not protect SN neurons from toxicity, unlike previous studies in cortical neurons and microglia. The trend in behavioural data suggests a potential positive effect of wild blueberries in the MPTP model of PD.

\section{Simulation of acebutolol pharmacokinetics in chronic kidney disease (CKD) patients using physiologically based pharmacokinetic modelling (PBPK) \\ Shams Ismaeil, Paul Malik, and Andrea Edginton}

University of Waterloo School of Pharmacy, Kitchener, Ontario, Canada, N2G 1C5.

Background: Drug dosing in chronic kidney disease (CKD) today relies only on changes in the estimated glomerular filtration rate (eGFR), but CKD impacts multiple other body systems. On the other hand, PBPK uses known drug and physiological processes allowing extrapolation of dose in CKD patients based on healthy population data. Purpose: To evaluate the accuracy of a PBPK approach in predicting the pharmacokinetics (PK) of acebutolol in patients with varying degrees of renal impairment. Methods: Physicochemical and PK data were collected from literature on acebutolol and its metabolite, diacetolol. Healthy models were created using PK-Sim v8.0 and parameterized based on PK profiles in healthy subjects after oral and intravenous acebutolol administration collected from 5 clinical studies. The CKD model was then adapted from the healthy models based on changes in GFR, kidney volume, renal blood flow, hematocrit, plasma protein concentrations and gastric emptying time in CKD obtained from literature. The model was evaluated by comparing PK parameters to literature values. Results: The PBPK-predicted area under the curve (AUC) of acebutolol fell within a 1.33 error margin of the observed values in literature. Acebutolol AUC did not change in CKD patients. Diacetolol accumulated in impaired renal function, but our model was unable to predict its AUC within 1.33-fold error of observed values.Conclusion: The PBPK model of CKD was able to predict the plasma concentrations of acebutolol in renal impairment. PBPK is a valuable tool with great potential to be used to support dosing and clinical decisions in organ impairment populations.

\section{Development of liposomal formulation for delivering the Feldan Shuttle, a cell-penetrating peptide}

Marie-Noël Boulianne, Caroline Quéant, Karine Greffard, and Nicolas Bertrand, Faculty of Pharmacy, CHU de Quebec Research Center, Laval University

Background The Feldan Shuttles are cell-penetrating peptides with the capacity of binding to biopharmaceutical agents to facilitate their access to intracellular targets, therefore increasing their efficiency. However, the Shuttle-biological agent complex is unstable following systemic administration. This phenomenon could be prevented by the encapsulation of the complex in a nanovector, such as liposomes. Purpose The overall purpose is to evaluate de efficiency of liposomes to carry the cargo-Shuttle complex to the desired biological target in vivo while delaying the destabilization process. For this study, it was essential 
to develop, characterize and evaluate a liposomal formulation containing the Feldan Shuttle. Methods The synthesis of three types of liposomes (DOPC, DSPC, DSPG/DOPC) was accomplished by thin-layerhydration. The Feldan Shuttle was encapsulated by incubation and purified by ultrafiltration. The quantification of the encapsulated Feldan Shuttle was performed by colorimetric assay (BCA and micro BCA) and by HPLC. Results The Shuttle was successfully encapsulated within the three liposomal formulations, the DSPG distinguishing itself with the highest encapsulation efficiency $(24 \% \pm 0.04)$. The physical properties of the formulations were satisfactory maintaining a size $<200 \mathrm{~nm}$ and a PDI $<0.2$. Conclusion These results results confirm the capacity of liposomes to encapsulate the Feldan Shuttle, while preserving adequate physical characteristics. The results obtained will contribute to the development of a synergic vectorization technology, Shuttle-liposome, for the delivery of biological agents in vivo. 